\title{
XVII Canadian Congress of Neurological Sciences Pre-Congress Courses - June 23, 1982
}

\author{
COURSE I: "Neuro-Ophthalmology" \\ Chairman: J. A. Sharpe
}

\begin{abstract}
The course will review recent concepts of the physiology and disorders of gaze, nystagmus, vertigo, and the pupil in neurological diagnosis, in the morning session. In the afternoon, facial movement disorders, clinical and electrophysiological aspects of optic nerve disease, juxtasellar lesions, and cerebral visual disorders will be discussed. The faculty from the disciplines of neurology, neurosurgery, neurophysiology, ophthalmology, and otolaryngology will present clinical and laboratory aspects of these neuro-ophthalmic topics.
\end{abstract}

$\begin{array}{ll}\begin{array}{l}\text { Physiology of Eye Movements } \\ \text { Disorders of Gaze }\end{array} & \begin{array}{l}\text { R. David Tomlinson } \\ \text { Robert B. Daroff }\end{array} \\ \begin{array}{l}\text { Nystagmus and Saccadic Oscillations } \\ \text { Dizziness and Vertigo }\end{array} & \begin{array}{l}\text { James A. Sharpe } \\ \text { Hugh O. Barber }\end{array} \\ \begin{array}{l}\text { The Pupil in Neurological Diagnosis } \\ \text { Facial Dyskinesias }\end{array} & \text { James J. Corbett } \\ \begin{array}{l}\text { Optic Neuropathy: Clinical and } \\ \text { Electrophysiological Aspects }\end{array} & \text { Dan Boghen } \\ \begin{array}{l}\text { Sellar and Parasellar Syndromes } \\ \text { Neuro-ophthalmic Features and Management }\end{array} & \text { Trevor H. Kirkham } \\ \begin{array}{l}\text { Cortical Visual Dysfunction: Simultanagnosia } \\ \text { Prosopagnosia and Achromatopsia }\end{array} & \text { Harley S. Smyth } \\ \text { Questions and Answers } & \text { James J. Corbett }\end{array}$

\section{COURSE II: "New Answers to Old Questions in Current Neurological and Neurosurgical Practice" \\ Chairman: M. L. Schwartz and C. H. Tator}

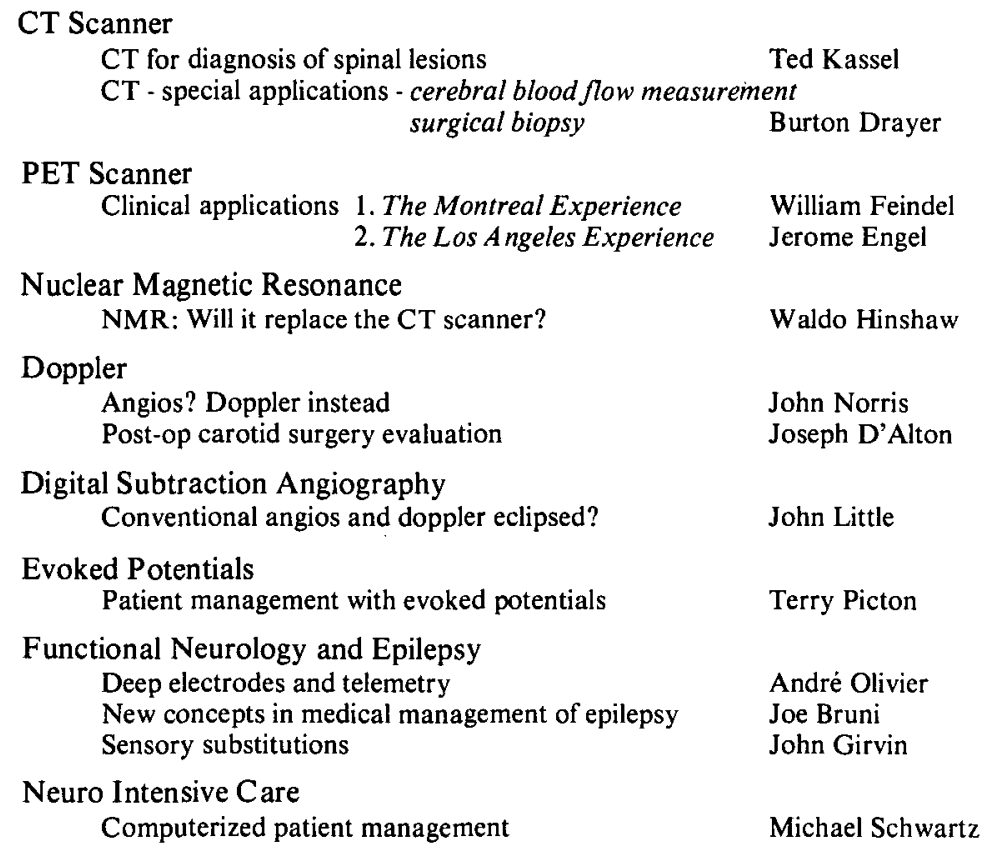


Adjuncts to Surgery

Laser: Space age technology in the operating room

Laser surgery - San Francisco experience

Cavitron for difficult tumors

Completing the eradication of malignant tumors interstitial radiation

Neville Russell Norman Chater Hart Schutz

Mark Bernstein

Overview

Neurology

Neurosurgery
J. C. Richardson

Claude Bertrand

\section{COURSE III: "The Stretch Reflex in Health and Disease" Chairman: Vernon B. Brooks}

Introduction: Reflexes in Motor Control

Stretch reflexes in posture and movement

What movement parameters are controlled?

Proprioception and Sensorimotor Cortex

Spasticity from spinal lesions

Spasticity and Rigidity from supraspinal lesions

Dysmetria, Tremor, and Cerebellum

General Discussion
Vernon B. Brooks

J. David Cooke

Richard B. Stein

John T. Murphy

Peter Ashby

Robert G. Lee

Vernon B. Brooks

\section{COURSE IV: "The Methodology of Clinical Research"} Chairman: V. C. Hachinski

\section{How to prepare a protocol: Steps $1-5$ \\ 1. Literature review \\ 2. Posing the research question \\ 3. Selecting a research design \\ 4. Selecting patients \\ 5. Specifying the intervention}

How to prepare a protocol: Steps 6 - 10

6. Specifying endpoints

7. Following patients and attributing endpoints

8. Sample size

9. Ethics

10. Budget

How to obtain research funds

How to execute a single center study

How to execute a multicenter study

Workshops
R. B. Haynes

M. Gent
H. B. Dinsdale
V. C. Hachinski
H. J. M. Barnett 


\section{SPECIAL LECTURES:}

1. PENFIELD LECTURE

"Cerebral Bypass Surgery, Review of 400 Cases: Current Status, Future Trends."

Dr. N. L. Chater, San Francisco

2. ROYAL COLLEGE LECTURE

"Overview of Current Problems in the Management of Cerebrovascular Disease - A Clinician's Viewpoint."

Dr. O. M. Reinmuth, Pittsburgh

3. PRESIDENT'S ADDRESS:

"Cushing's Disease: 50 Years Later."

Dr. Jules Hardy, Montreal

4. CANADIAN NEUROLOGICAL SOCIETY LECTURE

"Myoglobinuria - 1982."

Dr. L. P. Rowland, New York

5. McKENZIE AW ARD LECTURE

"The Effect of Cortical Lesions on Reflex Responses to Torque Perturbations in the Squirrel Monkey Forelimb." Dr. F. A. Lenz, Toronto

6. McNAUGHTON PRIZE LECTURE

"Heterogeneity of Myasthenia Gravis."

Dr. P. H. St. George-Hyslop, Toronto

POST-CONGRESS WORKSHOP - JUNE 26, 1982: "Halo Devices for Acute Spinal Injury" Chairman: C. H. Tator

The purpose of this workshop is to demonstrate the methods and value of halo devices and to encourage their use by Canadian neurosurgeons.

1. Patient Selection

a) Upper Cervical Injuries M. L. Schwartz

$\begin{array}{ll}\text { b) Lower Cervical Injuries } & \text { C. H. Tator }\end{array}$

2. How to Apply the Halo Ring

D. W. Rowed

3. How to Apply the Vest

V. E. Edmonds

4. Follow-Up Management and Removal

D. W. Rowed

5. Complications

C. E. U. Ekong

6. Organization of Halo Program

C. H. Tator 


\title{
XVII Canadian Congress of Neurological Sciences Abstracts of Scientific Program
}

\author{
Platform Presentations
}

1.

\section{A Pilot Study of the Calcium Antagonist Diltiazem in Migraine Syndrome Prophylaxis}

\section{R.J. RIOPELLE, J.L. McCANS, Kingston}

The pathogenesis of the migraine syndrome is felt in part to be related to accentuated vasoreactivity and a platelet aggregation/release response. Thus, any pharmaceutical preparation with effects to control these parameters might be of potential benefit in the migraine syndrome. Over the past decade, interest has grown in the potential clinical usefulness of a new class of pharmaceuticals known as calcium antagonists. The benzothiazepine derivative Diltiazem with vasodilatatory properties and effects on platelet aggregation has been used by one of us (JLM) in clinical trials for the control of symptoms in variant angina and in fixed coronary vessel disease.

A pilot study of the role of Diltiazem in patients with at least two migrainous events per week was carried out. Fifteen consecutive patients seen by one of us (RJR), and meeting standard criteria for the diagnosis of the migraine syndrome occurring at least twice weekly over the previous four weeks, were prescribed Diltiazem at $90 \mathrm{mg}$ per day per os in divided doses. Patients kept a weekly log of migrainous events, severity of the events, analgesic and ergot intake, and noted any side effects. One of us ( $R$ $J R$ ) reviewed each patient at four weeks where dose was adjusted if necessary, and at eight weeks, at which point the Diltiazem was discontinued.

All patients have tolerated Diltiazem without major side effects, and compliance as monitored by tablet count, has been high. Over the first four weeks of therapy, the weekly means of individual responses for percent decrease in events has been $37,54,79$, and 86 , respectively, while percent decrease in severity of events has been $44,41,43$, and 55 , respectively. A detailed analysis of patient response to 8 weeks of Diltiazem will be reported. The pilot study indicates that Diltiazem may be a useful and rapidly acting pharmaceutical for migraine syndrome prophylaxis. A single cross-over study of Diltiazem in migraine syndrome prophylaxis is currently underway.

\section{2.}

Familial Agenesis of the Corpus Callosum with Sensorimotor Neuronopathy: Further Clinical, Genetic and Epidemiological Observations

E. ANDERMANN, F. ANDERMANN, P. LANGEVIN, R. NAGY, D. BERGERON, J. LAROCHELLE, Montreal, Québec,

Rimouski, Chicoutmi

We have ascertained 237 patients in 144 sibships affected with this syndrome, all originating from Charlevoix County and the Saguenay-Lac St. Jean region of Quebec. The main clinical characteristics are: complete agenesis of the corpus callosum, mental retardation, sensorimotor neuropathy, scoliosis, and dysmorphic features. In infancy, affected individuals are areflexic and hypotonic, especially in the lower extremities. Postural and action tremor is often present in the first decade. Retardation is moderate and non-progressive. Initial progress in motor ability to the level of standing or walking with crutches at four to six years of age, is followed by motor deterioration, with most patients confined to a wheelchair by the early teens. Some patients develop a florid hallucinatory psychosis in the second decade. By the third decade, extreme scoliosis and severe dis- tal contractures are present, and most patients die in the third or fourth decade.

Several patients with all other features of the disease have a radiologically normal corpus callosum. This might suggest that the basic genetic defect responsible for the neurological disease interferes with the development of the cellular components of the corpus callosum.

Segregation analysis by several methods was compatible with autosomal recessive inheritance. Founder effect has been demonstrated by genealogical studies. The incidence of the condition in the region is estimated as $1 / 2500$ live births, with a carrier frequency of $1 / 25$.

Recognition of the syndrome has led to earlier diagnosis and prevention of recurrence within sibships. Although carrier detection is still unavailable, prenatal diagnosis by ultrasound may be feasible.

3.

\section{Risk of Multiple Sclerosis in Isolated Optic Neuritis}

T.E. FEASBY, G.C. EBERS, London

The risk of developing multiple sclerosis (MS) following clinically isolated optic neuritis (ON) is between 13 and $85 \%$ according to recent studies. To help determine the risk prospectively, we have investigated 36 patients with $\mathrm{ON}$ ( 5 had bilateral $\mathrm{ON}$ ) using visual evoked responses (VERs), auditory evoked responses (AERs), transpontine blink reflexes (BR), and high volume contrast-delayed CT scans (CT) to look for dissemination of lesions in the nervous system, and spinal fluid protein electrophoresis to look for oligoclonal banding (OB). The abnormalities found were: $6 / 31$ abnormal VER in the opposite eye (in unilateral $O N$ ) $7 / 35$ AER; $5 / 34$ BR; 0/9 CT; and 18/33 OB. Overall $27 / 36$ (75\%) had at least one clinically unsuspected abnormality. Interestingly, $50 \%$ of the OBnegative patients had clinically-silent lesions shown by electrophysiologic tests.

The high frequency of electrophysiologic and CSF abnormalities in these patients with clinically isolated ON favours the higher estimates for the risk of development of MS in this patient group.

4.

\section{Visually Evoked Potentials and Color Vision in Multiple Sclerosis}

\section{A. HARRISON, W. BECKER, W. STELL, Calgary}

Pattern Shift Visually Evoked Cortical Potentials (VEP) and color vision with the Farnsworth Munsell 100 Hue Test were assessed on the same day in twenty patients with mild Multiple Sclerosis (M.S.). Patients were selected on the basis of a prolonged $P_{1}$ latency in one eye and a normal latency in the other on a previous VEP, and screened for congenital color vision deficits with pseudo-isochromatic test plates.

In our study the VEP was normal in $16 / 40$ eyes $(40 \%)$ and delayed in $24 / 40$ eyes $(60 \%)$. Color vision was normal in $24 / 40$ eyes $(60 \%)$ and abnormal in $16 / 40$ eyes (40\%). Results of the VEP and 100 Hue were correlated in $21 / 40$ eyes $(52.5 \%)$; both tests were normal in 11 eyes and both abnormal in 10 eyes. Of the remaining $19 / 40$ eyes $(47.5 \%)$, the VEP latency was abnormal in 14/40 eyes with normal color vision, and color vision was abnormal in $5 / 40$ eyes with normal VEP latencies. The VEP may have shown more abnormalities than the color vision testing because patients were selectedon the basis of prolonged VEP latency. Considering this, the demonstration of visual abnormalities with color vision testing 
which were missed by the VEP is especially interesting.

These findings suggest VEP's and color vision tests assess different aspects of visual function and both may be useful in the diagnosis and assessment of patients with M.S.

\section{5.}

Check vs. Diamond Pattern Reversal: Orientation-Specific V.E.P. Deficits in Multiple Sclerosis

\section{S.G. COUPLAND, T.H. KIRKHAM, Montreal}

Checkerboard pattern reversal visual evoked potentials (VEPs) have been found most useful in confirming optic nerve disease in patients with clinically definite or possible multiple sclerosis (MS). Recent evidence of orientation-specific contrast sensitivity loss and the presence of orientationspecific VEP deficits in MS patients has been obtained using vertical sinusoidal gratings as stimuli. This study reports the presence of orientation-specific VEP delay in MS using the conventional checkerboard pattern presented in two orientations: normally oriented (check condition) or diagonally oriented (diamond condition).

105 clinically definite MS patients and 44 normal controls had VEPs recorded in an 11 degree circular test field containing check patterns (checksize $=30^{\prime}$ ) at $80 \%$ contrast. VEPs were recorded over $\mathrm{O}_{1}$ and $\mathrm{O}_{2}$ referenced to linked mastoids. Peak latency values of the N70 and P100 components were statistically analyzed using appropriate ANOVA and nonparametric statistics.

As a group, the MS patients showed significant VEP delays under both check and diamond pattern conditions. However, individual subject analysis revealed that about $20 \%$ of the MS population show VEP delay to only one pattern orientation. It was shown that by including a diamond pattern condition the diagnostic yield of VEP delay in these clinically definite MS patients was increased by $11 \%$ over that obtained with check stimulation alone rendering the additional 5 minutes testing time worthwhile.

6.

\section{Topographic Evoked Potentials: Test of Cortical Function in Cerebral Lesions}

\section{P.K.H. WONG, R.E. RAMSAY, Vancouver, Miami}

Current radiographic tests (angiography, radionuclide and CT scans) provide structural diagnostic information in patients with cerebral lesions. Cerebral blood flow (CBF) and positron emission tomography (PET) yield some information of cerebral function but are invasive. There is need for simple, non-invasive tests of cerebral function to complement clinical examination.

We studied patients with cerebral infarcts and tumors using 20 simultaneous electrodes to record $512 \mathrm{~ms}$ of EEG following visual stroboscopic, and median nerve stimulation. Data was acquired, averaged, and topographic video maps generated on-line by a microcomputer. Sequential maps were generated at $2 \mathrm{~ms}$ intervals, and can be viewed as an angiography-like movie, with voltage values converted to shades of grey, proportional to voltage.

Data to date from over 20 patients have been encouraging. Such evoked potential scans correlated well with clinical localization of lesions, while CT tended to underestimate the lesion size. Our experience indicates that this method provides additional information and suggests it is a suitable and sensitive test of cortical function. Patient and animal material will be presented, compared to CT and PET scan data.

\section{7.}

\section{Electrophysiological Investigations of the Sensory Systems in the Hereditary Ataxias}

\section{M.J. TAYLOR, W.J. LOGAN, Toronto}

The nature and incidence of sensory involvement in the hereditary degenerative ataxias is only recently being well investigated, and visual and somatosensory abnormalities have been reported. We previously studied auditory brainstem responses (ABRs) and cortical auditory evoked responses (AERs) in a series of Friedreich's ataxia patients who varied widely in age and degree of clinical involvement. The ABRs were markedly abnormal in all but the youngest patient and abnormalities occurred as a function of the severity and duration of the disease. AERs were recorded in most of the patients despite the extremely abnormal responses in the more caudal portion of the auditory system. The latencies of the AERs, however, were significantly longer in the Friedreich's ataxia patients compared to normal controls, suggesting cortical as well as peripheral involvement of the auditory system.

More recently ABRs, AERs, visual evoked responses (VERs) and somatosensory evoked responses (SERs) are being evaluated longitudinally in a series of children with various spino-cerebellar degenerative disorders with the emphasis on Friedreich's ataxia and ataxia telangiectasia patients. In the latter group ABRs are found to be normal in terms of both morphology and latencies in sharp contrast to the characteristic loss of waves in the ABR from Friedreich's ataxia patients. The VERs, however, in ataxia telangiectasia are consistently abnormal in the older patients, and there are significantly increased latencies when the responses can be obtained. The VERs recorded from the Friedreich's ataxia patients are more variable, consistent with earlier reports in the literature. In both of these groups of patients there are as yet no visual symptoms and their vision is considered normal. Preliminary work with the SERs appears to demonstrate that these may reflect the progression of the disease in ataxia telangiectasia patients. Other ataxia patients have patterns of results on these evoked potential measures which appear disparate from the above two groups.

The different profiles of the hereditary spino-cerebellar degenerative diseases on these electrophysiological tests may be an aid in the differentiation and physiological monitoring of these patients.

8.

\section{Anticholinergics in Adult-Onset Focal Dystonia}

\section{A.E. LANG, M.P. SHEEHY, C.D. MARSDEN, London, England}

The pathophysiology of the idiopathic dystonias is unknown and treatment remains unsatisfactory. Parenteral anticholinergic drugs usually cause rapid resolution of neuroleptic-induced acute dystonic reactions and high dose anticholinergics may improve dystonia in children. Anticholinergics remains one of the commonest treatments used in adult-onset focal dystonias although their general efficacy has not been proven. We investigated the effects of intravenous administration of atropine, benztropine and chlorpheniramine, in comparison to normal saline, in 20 patients with adult-onset focal dystonias (spasmodic torticollis (9) cranial dystonia (6), writer's cramp (5)) and retrospectively reviewed the notes of 71 patients (spasmodic torticollis (38), cranial dystonia (22), writer's cramp (11)) who had received oral anticholinergic therapy in varying doses at some time in the past. Patients with spasmodic torticollis and writer's cramp showed no consistent change with any of the intravenous drugs while the few with cranial dystonia who improved usually did so at the expense of druginduced sedation. The natural variability of these disorders was evident in the response to normal saline, which caused changes in the scores for severity of the involuntary movements of $20 \%$ or more in some patients. Only 6 of the patients who had received oral therapy were said to have obtained more than mild benefit from these medications and in 3 of them this improvement was transient.

We conclude that cholinergic mechanisms are not of prime importance in the pathogenesis of these conditions. However, each new patient warrants a trial of anticholinergic therapy in view of the low incidence of severe side-effects, compared to neuroleptics, and the occasional useful response obtained with these drugs.

9.

\section{Cerebral Glucose Metabolic Responses to Anoxia in the Newborn Rat}

\section{D.C.N. HOWSE, Kingston}

Hypoxia during the perinatal period is a common cause of permanent brain damage. In the term infant, the cerebral cortex and pyramidal cell 
layer of the hippocampus are particularly vulnerable. Cerebral glucose metabolism following hypoxic insult was studied in newborn rats (1-6 days) using the [14C]-2-deoxy-D-glucose technique. Hypoxemia was created by allowing the rat pups to breath pure nitrogen for 10 minutes to 6 hours later.

These qualitative studies show the pyramidal cell layer of the hip pocampus, and thalamic nuclei to be the metabolically most active regions in the newborn rat brain. Following anoxia, the metabolic activity was depressed in both regions for about 2 hours, after which a normal pattern was again seen. In a few pups a marked glucose hypermetabolism of the cortex was present following the insult. These findings provide evidence of impaired energy metabolism corresponding to known patterns of brain damage in the anoxic infant.

10.

\section{Microsurgical Treatment of Hemifacial Spasm}

\section{H.S. SMYTH, Toronto}

The mechanism of production of hemifacial spasm remains obscure, and its surgical treatment controversial. The symptom-complex of progressive involuntary unilateral facial myoclonus and synkinesis has been studied in ten patients, and recorded cine-photographically. Hemifacial twitching was stopped in all seven patients who underwent posterior fossa microsurgical exploration, the period to complete remission varying from one day to four months. Obvious arterial compression was discovered in all patients at various sites along the intracranial course of the VIIth nerve from pons to porus, two vessels being involved in most cases. Operative technique consisted of microdissection of the VIIth-VIIIth nerve complex, and has evolved to include Vlith nerve neuropexy using a silicone foam cradle and a Teflon felt cap. The first patient had a type 1 Chiari malformation requiring additional complete posterior fossa decompression. Neurological complications were confined to ipsilateral deafness in one patient, a $25 \%$ hearing loss in another, and transient facial paresis in a third patient.

Hemifacial spasms were not pulse related, nor was there any correlation between duration of symptoms and time to remission. While microvascular manipulation provides successful treatment for hemifacial spasm, the reasons for its effectiveness remain obscure and require further study.

\section{1.}

\section{Capsular Stimulation for the Thalamic Syndrome}

\section{I.M. TURNBULL, Vancouver}

Many patients with thalamic syndrome find the pain that persists while they rest disagreeable, but a lesser problem than severe dysesthesia that occurs with activity. The involved hand may become useless despite the preservation of motor function and adequate sensation.

Four patients have had electrodes implanted lateral to the involved thalamus. All have responded favourably. Both components of the problem, the steady pain and the dysesthesia, have proved amenable to stimulation, typically of ten minutes duration two or three times a day. Results, including incomplete maps of the internal capsule derived from step-wise intra-operative stimulation will be discussed.

\section{2.}

\section{Percutaneous Spinal Cord Stimulation: Indications and Results}

\section{R.G. VANDERLINDEN, Toronto}

Diagnostic percutaneous spinal cord stimulation for the treatment of chronic intractable pain was carried out on 112 patients. The patients were divided according to the etiology of their pain as follows: Failed Back Syndrome (67), Deafferentation Pain (33) and Miscellaneous (12). The Failed Back group was subdivided into A rachnoiditis (28 patients), Nerve Root Fibrosis (24), and Disc Degeneration or Undetermined (15). The Deafferentation group was subdivided into Peripheral Neuropathy (14 patients), Spinal Cord Injury (11) and Post-Amputation (8). In the Miscel- laneous group were lower limb crush injuries (4 patients) and malignancy.

Since April 1976, thirty-five patients have undergone implantation of permanent devices. The overall success rate was $66 \%$, with no difference between W.C.B. and non-W.C.B. patients. Half of the failures, occurring early in the series, were related to electrode migration or breakage and are now readily correctable. Five patients report feeling stimulation but no pain relief. Patients with nerve root fibrosis or peripheral neuropathy have an $80 \%$ probability of success, whereas only $50 \%$ of patients with arachnoiditis will respond to spinal cord stimulation. Patients with spinal instability, cord injury, post-amputation pain or malignancy have little chance of being relieved by this treatment.

\section{3.}

\section{The Use of Xenografts in Anterior Cervical Interbody Fusions}

\section{B.G. BENOIT, N.A. RUSSELL, M.A. FARIDI, Ottawa}

Cervical discectomies, using the techniques of Cloward or SmithRobinson, are usually followed by interbody fusion. Traditionally, autogenous bone from the iliac crest has been used. Not infrequently, such grafts are osteoporotic, thin, or imperfectly angulated.

We report a series of 173 consecutive patients, whose fusions were performed using bovine xenografts (Surgibone); the Cloward method was used in 107 patients, 79 with single level and 28 with two level fusions. The Smith-Robinson approach was used in 66 patients, 52 with single level and 14 with double level fusions. A total of 215 grafts were used in 173 patients.

These bovine grafts, which are composed primarily of hydroxyapatite, are available in cylindrical dowels or rectangular blocks. They are extracted with solvent to remove fat and blood, dried, and subsequently sterilized within hermetically sealed packages. The final product, is made of spongiosa, which is hard, uniform, and tailored accurately to fit the size required. These xenografts, do not possess osteogenic capacity or inductive potency. However, osteoconduction, splintage, and remodelling occur consistently. These grafts are immunologically inert.

In this series of 215 grafts, there were no cases of infection, fracture, or extrusion. One rectangular block collapsed in a two-level Smith-Robinson type fusion. There were no detectable adverse immunological reactions. In a follow-up period lasting up to six years, the only unusual radiological feature was a "halo" around the cylindrical dowels, which persisted until fusion had been achieved by the process of creeping substitution.

The use of this material facilitates either method of anterior discectomy and fusion, by providing a uniform hard graft, shortening operation time, and eliminating the morbidity associated with the donor site. This material is cost effective, because of a shortened postoperative hospital stay.

\section{4.}

\section{Preservation of Hearing in Patients Undergoing Excision of Acoustic Neuromas and other Cerebellopontine Angle Tumours}

\section{C.H. TATOR, J.M. NEDZELSKI, Toronto}

Microsurgical techniques have made it possible to identify and preserve the cochlear nerve from its origin at the brainstem to its course through the internal auditory canal in patients with small or medium size acoustic neuromas or other cerebellopontine angle tumours. In a consecutive series of 100 such tumours operated upon in the past six years we have attempted to preserve the cochlear nerve in 23 patients. The decision to attempt to preserve hearing was primarily based upon tumour size and the degree of hearing loss. In cases of unilateral acoustic neuromas these criteria were tumour size of less than $2.5 \mathrm{~cm}$. with apre-operative speech reception threshold of less than 50 decibels and a discrimination score of more than $60 \%$. In those patients who have bi-lateral acoustic neuromas or tumours of other type, the size and hearing criteria were significantly broadened. All patients were operated upon through the standard suboccipital approach.

In the 19 qualifying patients with unilateral acoustic neuromas hearing was preserved in six, although the cochlear nerve was preserved in 17 . Three patients had excellent post-operative hearing with three others hav- 
ing sound awareness and poor discrimination. With other angle tumours such as cholesteatomas and small meningiomas involving the internal auditory canal the results were better than with acoustic neuromas. In cases of bilateral tumours, every effort is made to save hearing irrespective of tumour size. This was accomplished in one of two such cases.

Over all, hearing preservation was accomplished in nine of 23 patients in whom this was attempted $(40 \%)$ with hearing acuity being equal to or better than the pre-operative level in six of these. Thus, it is worthwhile to attempt hearing preservation in selected patients with cerebellopontine angle tumours. In most instances this would require a suboccipital approach. It is certainly indicated in those with bilateral tumours.

\section{5.}

\section{Corrosion of Cranioplasty Plates}

\section{S.T. MYLES, D.D. COCHRANE, W.T. MURRAY, Calgary}

Thirteen patients had cranioplasties performed using stainless steel wire mesh and acrylic between 1974 and 1979.

Six patients had post-traumatic skull defects, four had bone flaps removed because of infection and three had tumor in the skull. There were eight males and five females with an age range from five months to seventynine years. Four patients have been lost to follow-up.

Seven of the remaining nine patients have shown gross or radiological evidence of corrosion of the prosthesis fifteen months to six years after insertion. The plate has been partially or completely removed from three patients.

Two children who had only stainless steel mesh implanted during the same five year period remain free of radiological change four years after insertion.

Metallurgical analysis of the stainless steel mesh has shown that it does not contain molybdenum and does not meet present standards for surgical implants. The addition of a methyl methacrylate coating to the stainless steel mesh has probably resulted in differential oxygenation and increased the tendency for corrosion of the wire mesh.

Cranioplasty using stainless steel wire mesh and acrylic has advantages over other methods. However, corrosion of the plate can occur as a late complication. Neurosurgeons should be certain that the stainless steel wire mesh used meets C.S.A. standards. The mesh should be completely coated with methyl methacrylate of uniform thickness to minimize corrosion.

16.

\section{Early Experience with the use of the $\mathrm{CO} 2$ Laser in Neurosurgery}

\section{N.A. RUSSELL, B.G. BENOIT, Ottawa}

The application of laser technology to various problems in clinical medicine has provided a new surgical instrument that utilizes high intensity beams of radiant energy to incise, coagulate, or vaporize tissue. In certain selected procedures it has proven so superior to conventional techniques that its use has become routine, e.g. photocoagulation for retinal detachment and diabetic retinopathy. The unique properties of laser energy give lasers the potential for becoming important tools in neurosurgery as well.

We have used the $\mathrm{CO} 2$ surgical laser combined with the operating microscope in the surgical treatment of ten patients with intracranial tumors and two with spinal cord tumors. This report will describe our experience.

The instrument is precise and accurate. Tumor debulking is possible with minimal brain or spinal cord retraction. The unit we use has certain limitations. Defocusing difficulties impede simultaneous coagulation of larger blood vessels and inadequate power (Wattage) makes large tumor removal time consuming. Thermal effects are minimal, allowing safe resection of lesions adjacent to important neurovascular structures. Sterilization of meningioma remnants is extremely efficient. There are also technical difficulties which stem from the necessity of preventing reflection of the beam from metallic retractors of suction tubes. The safety of both the patient and operating personnel must be constantly guarded.

We conclude that the laser is a valuable adjunct to the neurosurgical armamentarium, being particularly useful in dealing with lesions that are not readily amenable to removal by conventional surgical methods.
17.

\section{Intratumoral Autologous Mononuclear Cell Infusions in the Treatment of Recurrent Malignant Gliomas: A Phase 1 (Toxicity) Study}

\section{P. STEINBOK, W.J. THOMAS, C.L. DOLMAN, Vancouver}

Four patients with malignant gliomas recurring after surgical resection, radiotherapy and chemotherapy were entered into a trial of immunotherapy. Partial removal of the tumors was performed and autologous mononuclear cell infusions were given into the resection cavity via a subgaleal reservoir that had been inserted at the time of tumor resection. Mononuclear cells were obtained using a cell separator and Ficollhypaque separation techniques. Patients were followed carefully with clinical examinations, CT brain scans and immunological testing. Three patients have died and complete autopsies were done, looking specifically for evidence of mononuclear cell penetration into the tumor, and possible adverse effects on the normal brain or in the rest of the body. The results indicate that this type of therapy can be performed safely, and may be useful in the treatment of patients with malignant gliomas.

18.

\section{Stereotactic Biopsies of Cerebral Space Occupying Lesions}

\section{G. BOUVIER, P. COUILLARD, R. MALTAIS, J. LESAGE, F. ROBERT, Montreal}

Since the introduction of computerized tomography of the brain, early detection of cerebral lesions has been accessible to the clinician. This is particularly important when one considers lesions situated in major cortical areas or deep subcortical structures.

As direct surgical excision of these lesions is far from being innocuous, we have adopted the policy of obtaining first an accurate histological diagnosis by stereotactic biopsies. The main advantages of this technique are:

1. Possibility to define in space the shape and size of the lesion through multiple biopsies allowing the surgeon to propose a more rational treatment.

2. In cases of cerebral metastasis, possibility to know the site of origin without long and fastidious investigation in order to discover the primary lesion.

3. Relatively simple technique with a low complication rate.

Since 1979, we have performed stereotactic biopsies on 35 patients (a total of 160 biopsies). There has been no mortality. One cerebral hematoma was encountered. Less than 5 patients had a transitory aggravation of their deficit (mainly patients harbouring a prerolandic lesion). Definite histological diagnosis was not obtained in 2 cases.

Thus, cerebral stereotactic biopsy is a reliable, sensitive, and relatively innocuous technique. It is our feeling that it will become a major instrument in the investigation and treatment of cerebral space occupying lesions.

\section{9.}

\section{Pergolide in the Treatment of Parkinson's Disease}

\section{A.E. LANG, N.P. QUINN, C. THOMPSON, C.D. MARSDEN, S. BRINCAT, J.D. PARKES, London, England}

Forty-three patients with Parkinson's disease (4 previously untreated, 9 with lack or loss of response to levodopa, and 30 with response fluctuations) were given pergolide mesylate $0.2-17 \mathrm{mg}$ daily in addition to, or partially replacing, their existing therapy.

Twenty-one patients, receiving $1.2-17$ (mean 6.7 ) $\mathrm{mg} /$ day for $2-14$ (mean 9.7) months, obtained sustained objective or subjective benefit with pergolide. Fifty percent of the patients with fluctuations improved, but most of those with loss or lack of response to levodopa responded poorly to pergolide. No other features were useful in predicting outcome. Twentyfour patients discontinued pergolide, 8 primarily because of lack of useful response (mean dose $5 \mathrm{mg} /$ day) and 16 because of adverse effects. Fourteen of these 16 patients obtained no benefit before stopping pergolide 
(mean dose $2 \mathrm{mg} / \mathrm{day}$ ). Seven patients who had previously experienced psychiatric side-effects on other treatments stopped pergolide for this same reason. Five patients withdrew primarily because of an unacceptable increase in abnormal involuntary movements (mean dose $2 \mathrm{mg} /$ day).

Despite treatment failure in many cases, the addition of pergolide will benefit about $50 \%$ of late-stage parkinsonian patients who experience response fluctuations on their existing therapy.

\section{0.}

\section{“Drug Holiday" in Parkinson's Disease, - A Long Term Appraisal}

\section{O.S. KOFMAN, Toronto}

With the use of brief duration levodopa "drug holiday" therapy ie. complete withdrawal, the concept of an enhanced response and relief of disabling side effects in Parkinson patients has previously been assessed on a limited and short term basis without adequate follow up studies. (Sweet 1971, Direnfeld 1978, Weiner 1980) The need for further evaluation relative to possible lasting benefit has been suggested by Weiner 1980 , De Jong 1982.

Complete withdrawal of all levodopa compounds for a period of at least one week may influence receptor blockade by resensitizing and can alter denervation hypersensitivity. We have studied 20 Parkinson patients who have had complete levodopa drug holidays while in hospital. Each of these patients had developed either lack of response to medication or dose limiting side effects including dyskinesia, on-off phenomena or psychotic manifestations.

During the drug holiday further deterioration of mobility was noted. Upon restarting levodopa compounds, most but not all patients initially responded to smaller quantities of the previously used medication. Many of the drug induced side effects were eliminated or reduced. Three patients had additional drug holidays.

The follow up assessments of these 20 patients extended from months to 5 years. In most cases the beneficial effects obtained from complete levodopa withdrawal was generally limited to weeks or months with a subsequent recurrence of many of the pre-existing problems. Four of the 20 patients appeared to have a more prolonged beneficial effect. In some cases the improvement developed several months after the drug holiday and has been maintained.

These observations would suggest that "drug holiday" has a limited long term beneficial effect in a minority of patients who have developed problems associated with prolonged levodopa therapy. Further trial appears to be warranted particularly in intractable problems.

21.

\section{Dementia in Parkinson's Disease}

\section{R.L. TRITES, D. GRIMES, A. QUARRINGTON, A. KAPOOR, Ottawa}

Recent reports suggest that 40 to $50 \%$ of patients with a diagnosis of Parkinson's Disease (PD) are demented. The demented and non-demented groups may differ in etiology and in the course of the disease. Much is yet to be learned, however, about the nature of dementia in patients with PD and in particular whether the deficit resembles a dementia of the Alzheimer form or is more similar to the subcortical dementias.

This paper presents the results on 28 patients with documented PD ( 16 males \& 12 females) with an average age of 62.6 years, education of Grade 11.9 and Global IQ of 104 . All were English speaking and right-handed. The patients were rated by an experienced neurologist according to criteria such as duration of PD, severity, duration on Dopa, response to Dopa, degree of bradykinesia, degree of dyskinesia, clinical assessment of dementia, hallucinations and depression. All patients were given a dementia workup including CT scan, EEG and bloodwork. The patients also received an extensive neuropsychological examination which included measures of intelligence, academic achievement, memory and concept formation.

Statistical analysis produced three important patient profiles. The first profile to emerge contained the triad of clinical assessment of dementia,
EEG abnormality and presence of hallucinations. The neuropsychological deficits in this group were those typically described in early Alzheimer's patients. This included low Performance IQ, difficulty on visual/spatial tests, memory impairment and personality test signs of confusion. Of considerable interest was the high incidence of drug induced hallucinations in this group as opposed to the second profile. The second profile to emerge included the group with longer duration of the disease associated with test scores representing reduced efficiency on academic achievement tests, lowered fund of general information and lower Verbal IQ. It appears that patients with longstanding PD were not demented, but rather were less concerned with current affairs and new learning. In a third profile, CT measures (size of the third ventricle, sulci and lateral ventricle) tended to be highly associated with duration on Dopa and positive response to Dopa, but importantly were not correlated with clinical or neuropsychological test evidence of dementia.

Clinical signs of depression and depression test scores occurred independently of duration of PD, dementia and CT atrophy scores suggesting that depression in PD patients is more directly related to basic personality make-up.

\section{2.}

\section{Parkinson's Disease: Evidence for Vestibular Dysfunction}

\section{O.B. WHITE, J.A. SHARPE, J.A. SAINT CYR, Toronto}

Disordered postural reflexes are features of Parkinson's disease but no abnormality of vestibular function has been quantified. We studied the vestibulocular reflex (VOR) and its visual and voluntary (non-visual) control in 14 Parkinsonian patients and 10 age matched controls. Horizontal eye movements were recorded by infrared oculography and analyzed using a PDP-11 computer. The full system bandwidth was $0-200 \mathrm{~Hz}$. Patients underwent whole body oscillation in the yaw axis at frequencies from 0.3 to $1.0 \mathrm{~Hz}$. under five conditions: 1) darkness, 2) fixation of a stationary target, 3) fixation of an imagined stationary target, 4) fixation of a target moving with the head and 5) fixation of an imagined moving target in darkness. Conditions 3 and 5 test voluntary control of the VOR.

In advanced Parkinsonism, VOR activity was subnormal in darkness at all frequencies. In conditions 2 and 3, all patients raised their VOR gains (eye velocity/head velocity) to the normal range but gains were significantly below control group values. Visual cancellation of the VOR was impaired in condition 4. In advanced disease, VOR gains were paradoxically higher than the low gains recorded in condition I (darkness). In condition 5 , voluntary VOR cancellation was impaired; moreover, in advanced disease VOR gains were higher than in darkness. In darkness (conditions 1, 3 and 5), vestibular eye movements were punctuated by periods of wandering eye movements. Paradoxical elevation of VOR gain during visual and voluntary cancellation suggests structural integrity of the brainstem reflex pathway. The results indicate disordered voluntary and visual supranuclear control of the VOR, analogous to disordered supraspinal control of the spinal reflex arc in Parkinson's disease.

\section{3.}

\section{Electrophysiological Evidence of Improved Neuromuscular Transmission in Patients with Myasthenia Gravis Undergoing Plasmapheresis}

\section{J. DE LËAN, Québec City}

Plasma exchange combined with immunosuppressive drugs is an effective form of treatment in myasthenia gravis. Whether plasma exchange directly affects neuromuscular transmission remains controversial in the literature. This paper presents evidence of improved neuromuscular transmission following plasmapheresis in two patients with generalized myasthenia gravis.

Five consecutive plasma exchanges were performed every 5 to 6 weeks in both patients. Supramaximal repetitive stimulation were carried out before each exchange. In one patient, data collection was extended to 24 consecutive days. Muscle temperature was kept constant at $35^{\circ} \mathrm{C}$. Clinical status was assessed concomitantly. Anti-cholinesterase drugs were withheld following the last evening dose. 
1. Decrement did not change significantly in the hours following the first exchange.

2. Decrement was significantly reduced after the second or third plasma exchange.

3. Further improvement in neuromuscular transmission was recorded in one patient for 5 to 7 days after the fifth exchange.

4. Improvement in electrophysiological studies was accompanied by a concomittant decrease in muscle fatigue.

In the light of these data, plasmapheresis can alter significantly neuromuscular transmission in patients with myasthenia gravis.

\section{4.}

\section{The Efficacy of Azathioprine in Myasthenia Gravis}

\section{J.G. HUMPHREY, P. ST. GEORGE-HYSLOP, Toronto}

13 patients with chronic severe myasthenia gravis (MG) refractory to thymectomy and corticosteroids were followed for 24 months. Their clinical course (clinical score, number of relapses, courses of plasma exchange, steroid \pm pyridostigmine levels) and anti-acetylcholine receptor antibody (AChR Ab) titres were assessed for 12 months pre-azathioprine (control period) and for 12 months on Azathioprine $2.5-3.0 \mathrm{mg} / \mathrm{kg}$ body weight (treatment period). To obviate the effects of time the area under and the slope of each parameter time curve were compared for the control and treatment periods of each patient.

A significant reduction in the severity of MG (clinical difference: 10.69 $\pm 18.4, p<0.03$ ), the number of relapses per year (mean difference: 1.69 $\pm 2.02, \mathrm{p}<0.01$ ), a marked increase in the rate of improvement and reduced pyridostigmine requirements were found. There was no difference in steroid levels or anti AChR Ab titres between control and treatment periods. After 12 months of azathioprine, 8 patients were improved, 3 had become more stable and 2 were unchanged. Complications or adverse effects due to Azathioprine were minor in 12 of 13 patients. One patient developed a serious red cell aplasia.

Azathioprine appears useful in the treatment of chronic refractory MG.

\section{5.}

\section{Evidence for a Thymic Factor in the Pathogenesis of Myasthenia Gravis}

\section{C.W. OLANOW, A.D. ROSES, Durham}

Attention has focused on the acetylcholine receptor (AChR) antibody in the pathogenesis of myasthenia gravis (M.G.) because:

1. They are present in $90 \%$ of M.G. patients.

2. Passive transfer to animals results in an experimental M.G.

3. They accelerate degradation of AChR in tissue culture.

4. Reduction in antibody titre is associated with clinical improvement with plasma exchange.

We treated 47 consecutively presenting M.G. patients by total thymectomy according to a standarized protocol. Medical status was optimized prior to surgery by plasmapheresis when necessary. Drugs were stopped or avoided whenever possible. All patients improved and in the majority sustained benefit was attained without the need for medication. A reduction in AChR antibody titre was not essential for this improvement and there was no correlation between the antibody titre and the clinical status. No patient converted to a negative titre and many AChR antibody titres increased.

This dramatic improvement in clinical status following thymectomy without drugs and without a change in $\mathrm{AChR}$ antibody titre suggests that a factor produced by or within the thymus gland is essential for clinical weakness in M.G.
26.

\section{McNaughton Prize Paper}

\section{Heterogeneity of Myasthenia Gravis}

P.H. ST. GEORGE-HYSLOP, J.G. HUMPHREY, D.A.G. MICKLE, S. WONG, Toronto

It has been suggested the MG is a heterogeneous disorder, there perhaps being several subtypes of MG, each with anti-Acetylcholine Receptor $\mathrm{Ab}$ (anti-AChR Ab) of different specificity/affinity. We examined the possibility of heterogeneity within MG by analysing several clinical, immunological and pathologic parameters of MG in 88 patients with stable generalized MG. It was observed that patients differed in serum anti-AChR $\mathrm{Ab}$ titre, having either low $(<1 \mathrm{pMole} / \mathrm{ml})$ or high $\mathrm{Ab}$ Titres. This difference was not related to duration, or severity of $M G$, but did correlate with the distribution of MG, the prognosis and the thymic pathology. Additionally, patients with different forms of thymic pathology tended to differ in both clinical and immunologic parameters. When patients with similar thymic pathology (atrophy, thymoma, hyperplasia) and similar Ab titres (high/low) were grouped, all 6 subgroups differed in regards to several clinical and immune parameters including prognosis. These differences were distinct and suggested the presence of heterogeneity of MG. Classification of MG by both thymic pathology and serum anti-AChR Ab titre is likely to be clinically useful.

27.

\section{Failure of Barbiturates in Severe Head Injuries}

\section{K.S. POLYZOIDIS, J.D. McQUEEN, J. PINILLA, Saskatoon}

In 1980 a prospective study was undertaken in our institution in an attempt to evaluate the effect of barbiturates in closed head injuries. The patients were divided into two groups, Group A had a GCS 3-4 and Group $B$ had a GCS 5-8. Both groups received corticosteroids and mannitol. In addition Group A patients had continuous ICP and EEG monitoring, Swan-Ganz catheter and IV pentobarbitone.

The ICP course followed one of three patterns:

1. Immediate decrease and permanent normalization;

2. Initial reduction but subsequent progressive increase;

3. No initial response and continuous rise.

Application of the Glasgow Outcome Score showed: 16 Group A patients: 12 died, 1 in vegetative state and 3 severely disabled 18 months after the injury; 15 Group B patients: 2 died, 1 in vegetative state, 3 severely disabled, 3 moderately disabled and 6 with good recovery.

Obviously, the barbiturate coma, although usually affecting the ICP, failed to improve the outcome of the most severely head injured patients (GCS 3-4). However, it has a definite place in the reduction of ICP during the evacuation of acute subdural hematomas. It is possible that it is effective in children or adults with GCS 5-7.

28.

\section{Hockey as a Cause of Acute Spinal Cord Injury}

\section{C.H. TATOR, C.E.U. EKONG, D.W. ROWED, M.L. SCHWARTZ, V.E. EDMONDS, Toronto}

Although sports and recreational activities have been recognized as traditional causes of acute spinal cord injury, until recently hockey has not been a frequent cause. For example, in a series of 55 patients with acute cord injury due to sports-recreational activities which occurred between 1948 and 1973, not one was due to hockey. In contrast, between 1974 and 1981 we treated six patients with cervical spinal injury due to hockey, five of whom were seen during a 13-month period. Five of the six sustained an acute cervical spinal cord injury and all were males aged 15 to 26 years. Four of them struck the boards with the neck flexed and one struck another player with his neck flexed. The one patient without cord injury struck the boards with his neck extended. Three had complete cord injuries, two had complete motor loss and partial sensory loss, and one had a cervical root 
injury only. The commonest bony injury was a burst fracture of C5 or C6. One of the patients with a complete cord injury died three months later of a pulmonary embolus, and the other cord injuries have shown minimal improvement.

The reasons for the apparent increase of these major injuries due to hockey are unknown. A program to study this problem will be described.

\section{9.}

\section{Treatment of Acute Spinal Cord Injury using Steroids and Local Cooling}

\section{R.R. HANSEBOUT, Hamilton}

Laboratory studies have shown improvement in motor function following treatment of severe spinal cord injury using parenteral steroids and local cord cooling. These techniques were modified for human use.

In 15 patients with immediate complete sensorimotor paralysis below the level of the cord lesion, after informed consent, treatment using parenteral steroids and local cord cooling was administered. Unfortunately, due to technical problems involving transport and preoperative evaluation, local cooling was only begun at an average time of $81 / 2$ hours following injuries, deemed late according to laboratory studies. Nevertheless nearly one-half of treated patients recovered significant sensation, (1) $8 \%$ became ambulatory and only (1) $8 \%$ died of severe head injuries. This compared favourably with the results of conventional therapy where mortality and ambulation rates are respectively, $37 \%$ and $2 \%$.

In a review of 81 patients with complete cord injuries treated in various centres throughout the world, $57 \%$ of patients had neurological improvement, $12 \%$ became ambulatory and the mortality rate was reduced to $12 \%$.

In view of this encouraging trend, further trials of steroids administration and cord cooling begun as soon as possible (less than 6 hours) after injury are recommended.

\section{0.}

\section{Indications for Neurosurgical Intervention in Patients with Symptomatic Spinal Metastasis}

\section{R.G. PERRIN, K.E. LIVINGSTON, Toronto}

Symptomatic spinal metastasis is a serious complication of systemic cancer. Characteristically, pain is the earliest and most prominent symptom followed by weakness, sensory loss and sphincter dysfunction. The natural history is one of relentless progression to complete paraplegia with incontinence of bowel and bladder.

Therapeutic intervention is undertaken for relief of pain and restoration of neurologic function. Optimal treatment strategy involves a multidisciplinary approach with cooperation among neurosurgeon, oncologist and orthopedic surgeon. The indications for neurosurgical intervention are:-

1. radiation resistant tumor

2. radiation - saturated field

3. unknown primary

4. rapidly evolving or far advanced paraplegia

5. spinal malalignment or bony encroachment

Based on these criteria, we have undertaken neurosurgical treatment in 200 patients with symptomatic spinal metastasis. Pain relief achieved in $75 \%$ of patients; $66 \%$ were ambulatory following surgery (another $16 \%$ showed significant improvement in motor function); $43 \%$ enjoyed a "satisfactory" outcome - walking and continent 6 months following surgery.

Multidisciplinary cooperation and aggressive management can achieve gratifying results in patients with symptomatic spinal metastasis.
31.

\section{The Effect of Human Chorionic Gonadotropin (HCG) on Functional Recovery of Spinal Cord Sectioned Rats}

\section{A.A. PATIL, M.P. NAGARAJ, Minot}

The effect of Human Chorionic Gonadotropin (HCG) on functional recovery after complete spinal cord transection in the rat was studied. Twelve rats ( 6 male and 6 female) received HCG daily for the first week and then every alternate day for three weeks. Ten rats $(5$ male and 5 female) severed as control. Return of bladder function, Tarlov`s grading and measuring the maximum angle at which the rats were able to climb up an inclination were used as criteria in assessing the functional recovery. Improvement was noted in the HCG treated rats as compared to control group was very significant $(p<0.001)$. No difference in the recovery between the male and female rats were noted. No recovery what so ever was noted in the control group even at six weeks. The possible mode of action of HCG on spinal cord injury will be briefly discussed.

32.

\section{Regeneration of Long Spinal Axons in the Rat}

P.M. RICHARDSON, V.M.K. ISSA, A.J. AGUAYO, Montreal

In previous experiments (Nature 284, 264; Science 214, 931; Can. J. Neurol. Sci. 8,195$)$ it was demonstrated that many intrinsic CNS neurons with their cell bodies in the brain or spinal cord possess the capacity for axonal elongation into peripheral nerve grafts. However, no long descending spinal axons were seen to enter peripheral nerve grafts at the mid-thoracic level (Brain Research, in press). Here, peripheral nerve segments were grafted to the spinal cord at the low cervical level after section of one lateral column in 11 rats. The grafts were at least $1.0 \mathrm{~cm}$ long and were joined distally to the lower trunk of the brachial plexus. Five months later, the origin of axons in the grafts were identified after injection of horseradish peroxidase (HRP) into the grafts. In all 11 rats, labelled neurons were found in the brainstem $2.0-3.5 \mathrm{~cm}$ rostral to the grafts and also in low thoracic and lumber segments of the spinal cord, situated 4 . $5 \mathrm{~cm}$ caudally. The corticospinal tracts lie in the dorsal columns in rats and, therefore, the cerebral cortex was not surveyed in these experiments. Most of the labelled neurons in the brainstem were in the raphe and medullary reticular formation, lateral vestibular nucleus, ventrolateral pontine tegmentum and red nucleus. HRP-containing cell bodies in distal spinal cord segments were in Clarke's column, intermediate grey matter and ipsilateral and contralateral nucleus proprius. In further rats, extension of long descending spinal axons into cervical grafts was verified by radioautography after injection of tritiated leucine and proline into the brainstem. It is concluded that axons of most fibre tracts in the lateral columns of the rat spinal cord possess at least some regenerative potentiality. The reasons for the apparently different behaviours of descending spinal axons at cervical and mid-thoracic levels are unknown.

33.

\section{Polylysine Attached Serum Factors Enhance the Performance of Central Nervous System Neurons in Vitro}

\section{R.J. RIOPELLE, Kingston}

In embryonic neuronal development and in regeneration the matrix in which axonal elongation occurs is of increasing interest. Dissociated embryonic and neonatal neuron culture systems have been used to detect and quantitate trophic factors from many tissue sources. In these systems there is evidence to suggest that the uncharacterized factors can act directly, or indirectly by conditioning the matrix on which the neurons grow.

Falcon culture dishes were coated with poly-L-lysine $(0.1 \mathrm{mg} / \mathrm{ml}, 24 \mathrm{~h}$, $37^{\circ} \mathrm{C}$ ) and then incubated for 24 hours with calf serum at various concentrations from 0 to $100 \%$ in a defined synthetic culture medium. At 24 hours, the serum containing medium was removed and the plates washed extensively with serum-free defined medium. Dissociated neuron-enriched cells from 7 or 8 day chick embryo spinal cord or optic tectum were seeded 
at 50,000 to $1,000,000$ cells per $35 \mathrm{~mm}$ plate in serum-free defined medium. Thereafter, cell attachment and process length were measured microscopically.

At all times after 48 hours in culture, viability, attachment, and process length were significantly greater in serum-treated plates than in control plates and these parameters were saturable at low serum concentrations. Because of the enhanced attachment and increased process-to-process connection in the serum-treated plates, it is not possible to say what proportion of the trophic activity derived from the serum and what proportion derived from the cells by virtue of their interaction at any given time. However, below 500,000 cells per plate, the percent process-bearing cells per plate at 24 hours showed a plateau, suggesting that cell-cell interaction below this cell concentration is not an explanation for the differences between serumtreated and control plates. Physical and chemical characteristics of the polylysine-attached factors will be discussed.

The study of growth matrix conditioning using in vitro paradigms may provide many clues to axonal growth, sprouting, and regeneration in the central and peripheral nervous systems in vivo.

34.

\section{McKenzie Award Paper}

\section{The Effect of Cortical Lesions on Reflex Responses to Torque Perturbations in the Squirrel Monkey Forelimb}

\section{F.A. LENZ, W.G. TATTON, R.R. TASKER, Toronto}

Imposed displacement of many joints evokes an electromyographic (EMG) response consisting of early latency (M1) and long latency (M2) components, in muscles stretched by the joint displacement. Whereas previous studies have stressed similarities, the present study demonstrates significant differences in the EMG response of different muscles to imposed joint displacement.

Graded torque loads were applied about the metacarpophalangeal, wrist or elbow joint. The resulting EMG response in flexor digitorum profundus (FDP), flexor carpiulnaris (FCU) or short head of biceps (SHB) was computer sorted and averaged. After some trials, the $M$ response to supramaximal nerve stimulation determined, as a measure of maximal motorneuron output to the muscle being studied. In normals, the EMG response in FDP consists of an $\mathrm{M} 2$ component without an $\mathrm{M} 1$ component equivalent to that seen in FCU and SHB. These studies were repeated in both forelimbs of monkeys with unilateral, stimulation mapped lesions of the forelimb motor cortex. FDP M2 activity, expressed as a fraction of the $\mathrm{M}$ response, was decreased or absent contralateral to the lesion in all animals studied. In SHB M1 activity was significantly increased but M2 activity was not significantly altered with respect to the normal side.

These results indicate that the central and/or peripheral mechanisms generating M2 activity in FDP differ from those in SHB in terms of cortical dependancy.

\section{5.}

\section{A Demographic Study of Temporal Lobe Epilepsy: 174 Patients}

\section{M.W. JONES, Vancouver}

Considering the frequency of temporal lobe epilepsy (partial seizures with elementary or complex symptomatology), it is surprising there are not more clinical series describing the clinical features such as etiological factors, seizure types, EEG findings and outcome with treatment. Most studies have reported on the efficacy of new anticonvulsants or appropriately stressed the benefit of elective excisional surgery.

As a result, it seemed both of interest and of relevance to look systematically at a large series of patients studied by one person in a consecutive fashion. This study reports on 174 consecutive patients with this diagnosis seen between 1976 and 1981 from a private practise setting.

There were no major differences between males and females $(86 \mathrm{M}$, $87 \mathrm{~F}$ ). $59 \%$ were diagnosed by age $19.33 \%$ were diagnosed after the age of 30 . One could determine an etiology in $56 \%$ of cases; birth injury (19\%) being the commonest. In those over age 30 at onset, one was only able to find a cause in $44 \% .33 \%$ had significant neurotic problems of anxiety and depression and $10 \%$ had a major mental illness. $24 \%$ of the series had seen a psychiatrist. First degree family relatives had epilepsy in $14 \% .79 \%$ had epileptiform EEG's and 14\% were normal or dysrhythmic. $51 \%$ had "grand mal seizures" at some time. $69 \%$ had automatisms and $17 \%$ had grand mal seizures as their only seizure expression. An aura of taste or smell was rare at $6 \%$ and $4 \%$ respectively. Only $26 \%$ of patients had gone one year without a seizure and conversely, $22 \%$ of patients were having several per week.

As with large, multi-disciplinary university clinics that seem to attract the most refractory cases, the same sorts of problems exist for the private practising neurologist.

\section{6.}

\section{Lorazepam in Prolonged Seizure Activity in Children}

\section{R.E. MASOTTI, Kingston}

Although intravenous diazepam is regarded as the treatment of choice it has a short half-life and convulsions may recur after their initial supression. Serious side effects may occur such as apnea, hypotension and cardiac arrhythmias. Lorazepam is a new benzodiazepine derivative. Preliminary studies in 62 children from Europe and Brazil, and 3 children from North America, have suggested that it is a potent, safe and longer acting anticonvulsant.

This report will outline the experience with intravenous lorazepam in 28 episodes of prolonged seizure activity occurring in 8 children, aged 10 months to 17 years. Lorazepam was administered over 2 minutes in a standard dose of $0.05 \mathrm{mg} . / \mathrm{kg}$. diluted to $10 \mathrm{cc}$. with saline. None of the children had received prior parenteral anti-convulsants.

The average latency of the effect of I.V. lorazepam was 5 minutes. None of the patients suffered any adverse effects from the injection and the majority did not have the prolonged period of somnolence that characteristically follows intravenous diazepam.

Lorazepam was effective in controlling any observable seizure activity for over 12 hours in 18 of 28 episodes (65\%) with control for 6-12 hours in 6 other episodes $(21 \%)$. Only 4 injections (14\%) produced seizure control for less than 6 hours.

On the basis of this limited experience lorazepam appears to be a safe and effective drug with a duration of action longer than that achieved by diazepam. Details of the individual cases will be presented.

\section{7.}

\section{The Offspring of the Epileptic: Reproductive Loss, Physical and Mental Development}

\section{E. ANDERMANN, L. DANSKY, D. WILLIS, F. ANDERMANN, Montreal}

Although an increased frequency of major and minor malformations in offspring of epileptic women, particularly those taking anticonvulsant medication during pregnancy, is now established, other abnormalities during pregnancy, in the neonatal period, and in subsequent physical and mental development are less well recognized.

In a retrospective study of 100 epileptic women and 100 epileptic men, induced abortions and perinatal deaths were significantly increased in offspring of epileptic women as compared with offspring of epileptic men, and with the general population $(6.0 \%$ vs. $0.0 \%$; and $6.2 \%$ vs. $1.0 \%$, respectively). The spontaneous abortion rate did not differ significantly between the two groups $(12.6 \%$ vs. $14.2 \%)$, or from the general population, but was higher in offspring of women who had taken anticonvulsant medication during pregnancy $(22.8 \%$ vs. $10.0 \%)$.

In a prospective study of offspring of treated epileptic women, the mean gestational age and mean birth weight, as well as the condition of the liveborn offspring at birth, did not differ significantly from the general population. However, there was a significant increase in small for gestational age infants. The offspring were followed at regular intervals for over three years. Head circumference, length and weight were measured at each visit, and were correlated with the Griffiths Developmental Scale. Although the somatic parameters tended to be 1-2 standard deviations below the mean, 
the Griffiths Developmental Scales were at or above the mean in all age groups.

For many of the abnormal outcomes reported here, as for major and minor congenital malformations, it is difficult to distinguish among a number of confounding etiological variables, such as the effects of maternal epilepsy, genetic predisposition, drug effects, and socio-economic factors. However, our data suggest an mportant influence of the anticonvulsant medication.

38.

\section{Hypermetabolism of Phenytoin as a Cause of Treatment Failure}

\section{L.H. LEBRUN, J-P VILLENEUVE, Montreal}

Phenytoin hypermetabolism is not frequently recognized as a cause of treatment failure. We report the case of a 36 year-old male in whom pharmacokinetic studies demonstrated an increased metabolism of phenytoin and who required large doses of phenytoin to achieve seizure control.

This $74 \mathrm{~kg}$ man, developed focal right hemispheric seizures following a car accident in 1973. The administration of phenytoin $300-400 \mathrm{mg}$ daily never produced plasma levels above $5.0 \mu \mathrm{g} / \mathrm{ml}$. Inspite of several trials of phenobarbital, primidone, carbamazepine and valproic acid, with normal levels of these drugs, he sustained four to five seizures a week. Although the low phenytoin levels were thought to be due to poor compliance, the normal levels of other anticonvulsant drugs made us question this assumption and pharmacokinetic studies were carried out. Following an i.v. phenytoin infusion of $300 \mathrm{mg}$, his total plasma clearance was $0.0615 \mathrm{~L} / \mathrm{kg} /$ hour (normal $0.022 \mathrm{~L} / \mathrm{kg} / \mathrm{hr}$ ). Systemic availability was $74 \%$ (normal $70 \%$ ). Individual Michaelis-Menten parameters for phenytoin metabolism were estimated using the method of Ludden et al. The $\mathrm{Km}$ value was $4.64 \mu \mathrm{g} / \mathrm{ml}$, a normal value, while the $V \max$ of $14.0 \mathrm{mg} / \mathrm{kg} /$ day is above the normal range. The clearance of antipyrine and the breath elimination rate constant of aminopyrine, two model drugs metabolized by the liver, were also measured. His clearance of antipyrine was $115 \mathrm{ml} / \mathrm{min}$, (normal value 38 $\mathrm{ml} / \mathrm{min})$. His breath elimination rate constant for aminopyrine $\left(0.43 \mathrm{hr}^{-1}\right)$ was also faster than that of normal controls $\left(0.27 \mathrm{hr}^{-1}\right)$. Rapid metabolism of antipyrine and aminopyrine suggest that the abnormality of phenytoin metabolism in this patient is not limited to hydroxylation reactions, but is rather the reflection of a nonspecific induction of the liver drug metabolizing enzymes. Following these studies, the dose of phenytoin was increased to $800 \mathrm{mg} / \mathrm{day}$; his plasma level is now $15 \mathrm{ug} / \mathrm{ml}$ and he has had only two seizures over the last 20 months.

Low plasma phenytoin levels should not be systematically ascribed to poor compliance. In some patients, increased phenytoin metabolism can be a cause of low plasma levels and treatment failure. With the help of simple pharmacokinetic principles, this condition can be recognized and the optimal phenytoin dosage can be determined.

\section{9.}

\section{Surgical Treatment for Intractable Childhood Onset Generalized Seizures}

\section{J.A. WADA, G.B. THOMPSON, P.D. MOYES, Vancouver}

Early childhood onset seizures of organic cause are often refractory to the available medical treatment. A complicating factor for consideration of surgery is that many of these unfortunate patients have in addition, associated mental retardation. It is the purpose of this presentation to describe the results of alternative surgical intervention on 7 patients with a variety of childhood onset intractable generalized seizures and mental retardation who required continuous care and attention on the verge of institutionalization.

1. Localized excision for tonic flexion spasm or astatic-myoclonic seizures.

All four patients had infantile hemiplegia. These patients underwent localized frontal, frontocentral, rolandic or rolandic and temporal excision. Except for immediate postoperative period, there has been no recurrence of habitual seizures during the follow-up period of 1-3 years on continued medication.
2. Anterior callosal bisection for atonic seizures.

All 3 patients had frequent seizures with resultant repeated injuries. They all showed some electroclinical evidence of vaguely lateralized lesional processes and they underwent anterior $2 / 3$ callosal bisection. One patient remains entirely seizure free for the past four years, another patient has only infrequent head-nodding without loss of consciousness or fall, and is no longer sensitive to startle stimuli and the third patient has a significant reduction of seizure intensity and frequency (20-30/month - 3-4/month).

All these patients no longer require continuous care and remain functional within their families. Our experience suggests that (1) some intractable childhood onset generalized seizures are amenable to excisional surgery or partial callosal bisection and that (2) atonic seizure can occur as a result of bihemispheric ictal participation through the corpus callosum.

40.

\section{Crossover Comparison of Valproic Acid Versus Zarontin in Petit Mal}

\section{A.R.M. UPTON, L. McDONALD, Hamilton}

A crossover trial of Zarontin or Valproic acid for the treatment of previously untreated petit mal in nine patients (M 3, F 6; Age 6-12; mean 10 yrs. Follow-up, five months to 2 years) included split-screen videomonitoring and a bilateral alternating motor task (BAMT) for assessment of efficacy. Clinical examination, school performance, level of alertness and reports of subjective symptoms provided additional measures of drug effect. Nine blood levels over eight hours at every phase of treatment and random levels during other visits have been correlated with the EEG and performance tasks. There were major fluctuations in blood levels of both drugs during a 24 hour period and each child showed optimal performance at different blood levels.

Although each child showed greater benefit with one drug, there has been no measurable difference in overall effect of these drugs on seizure frequency, motor performance, photic response, side effects and EEG changes but conscious level, school performance, mood and behaviour were better on Valproic acid. Two patients suffered either grand mal or complex partial seizures on Zarontin, but not on Valproic acid. Three patients are free of seizures.

Further studies include a trial of both medications in those with persistent seizures.

\section{1.}

\section{Ventricular Widening in Childhood Bacterial Meningitis}

\section{R.D. SNYDER, Albuquerque}

Between 1975 and 1980,98 cases of childhood bacterial meningitis were seen. CT scans were performed in those cases which showed significant depression of consciousness, seizures, persistent full fontanel. focal neurologic signs, and persistent fever. Forty-nine cases had CT scans performed at least once. Ventricular widening was found in 11 cases. Five cases had persistent or increasing ventricular widening without clinical evidence of increased intraventricular pressure. These five cases showed no excessive increase in head circumference, no full fontanel, normal intraventricular pressure recording ( 1 case), and continued ventricular widening on CT during external ventricular drainage ( 2 cases). Other cases appeared to have elevated intraventricular pressure and more traditional pressure hydrocephalus.

Ventricular widening in acute childhood bacterial meningitis does not necessarily indicate increased intraventricular pressure in all cases: nor does ventricular widening necessarily regress with drainage of the ventricular system. Factors other than pressure must play a part in the change in cerebral mass. The process appears to be a diffuse atrophic encephalopathy. The mechanisms by which these acute changes are induced is unknown. Possible explanations include a vasculitis. bacterial neurotoxins, a toxic component of granulocytes, or an immunologic reaction.

Ventricular widening in childhood bacterial meningitis is not always an indication for surgical intervention. Follow-up over a period of time is appropriate looking for other signs of increased intracranial pressure. These other signs include persistent full fontanel, spread of sutures, increasing head circumference, and recording of elevated intracranial pressure. 
42.

\section{The Changing Pattern of Pseudo-Tumor Cerebri (PTC) in Childhood}

\section{P.R. CAMFIELD, J.A.R. TIBBLES, R. COUCH, Halifax}

Most information regarding PTC in children is extrapolated from adults. Thirty-seven cases of PTC have been seen at the I.W. Killam Hospital for Children in the past 12 years. All diagnoses were confirmed by elevated CSF pressure or severely bulging fontanelle resolving on follow-up. Unexpected presenting findings included neck pain (3), 4th and 7th nerve palsies, cerebellar ataxia (2). Three patients had CSF pleocytosis. Nineteen were $<6$ years of age with an etiologic diagnosis made in 13; refeeding after deprivation (6), immediately after treatment for cystic fibrosis (4), severe iron deficiency (1), systemic absorption of topical steroid (1), trauma (1). Nine were age 6-11 years with a cause assigned in 4 ; sinus thrombosis (2), topical steroids (1), post irradiation syndrome (1). Nine were age 11-14 years with no etiologic cause found in this group.

Only $3 / 37$ required LP shunt following failure of medical management which was observation or repeated LP's in 20 , or steroids \pm diuretics in 17. Two had significant permanent visual impairment - both referred late.

In conclusion, in children $<11$ years old, an etiologic diagnosis can often be made while, in older children, identifiable causes are rare. Traditional causes such as hypervitaminosis, outdated tetracycline or lead poisoning are now unusual, and otitis media is an uncommon cause in the modern era. PTC appears benign if treatment is initiated early.

\section{3.}

\section{Differential Diagnosis of the Floppy Newborn: The Value of Thin Ribs on Chest Radiograph}

\section{J.P. OSBORNE, E.G. MURPHY, A. HILL, Toronto}

In infants with neuromuscular disease, severe muscle weakness following delivery may cause respiratory difficulties and hypoxic-ischemic cerebral injury. This in turn may contribute further to hypotonia and thus obscure the primary diagnosis of neuromuscular disease. Historical features such as decreased fetal movements and polyhydramnios together with physical signs such as joint contractures indicate neuromuscular disease. The sign of thin ribs on chest $\mathrm{x}$-ray has been reported in congenital myotonic dystrophy. However, the specificity and incidence of this observation are unknown. In this presentation we review the prevelance of this radiological finding in a variety of neonatal neuromuscular diseases.

Over a five year period, 26 infants with neonatal neuromuscular disease were reviewed. The diagnosis was made by a combination of electromyography, muscle biopsy and examination of the mother where appropriate.

Thin ribs on chest $\mathrm{x}$-ray were observed in three of seven cases of con genital myotonic dystrophy, two of four cases of myotubular myopathy and one of five cases of Werdnig-Hoffman disease. Rib size was normal in one case of nemaline myopathy and three cases of non-specific myopathy. In six cases no chest $x$-ray was available.

The observation of thin ribs on chest $\mathbf{x}$-ray in the newborn strongly suggests neuromuscular disease. However, it does not occur invariably in, nor is it specific for congenital myotonic dystrophy. When seen in association with hypoxic-ischemic encephalopathy, it suggests that neuromuscular disease is the primary diagnosis.

\section{4.}

\section{Supratentorial Astrocytomas Grades III and IV in Children}

\section{R. DE LEO, D. FULTON, B. WEIR, Edmonton}

Twenty-two cases of malignant supratentorial astrocytomas occurring in children 16 years of age or younger over a 20 year period were analyzed. The mean age of presentation was 7.9 years with even distribution over the age groups and a 1.7:1 male to female ratio. A mean of 2.5 months was found from onset of symptoms to diagnosis with $10(45 \%)$ of them presenting symptoms within a month or less. Headache $(50 \%)$, vomiting
(63\%), papilledema (45\%) and motor deficit $(22 \%)$ were the most common findings.

Over half of the neoplasms were localized to the frontoparietal lobes with no predilection as to side. High incidence of abnormalities were seen on plain $x$-ray films of the skull $(63 \%)$. Eighteen children $(81 \%)$ underwent craniotomy. In 13 cases $(58 \%)$ subtotal removal was achieved. The rest had needle biopsy. Surgical outcome showed no intraoperative deaths, 2 children $(9 \%)$ were severely disabled, 1 child $(5 \%)$ was moderately disabled, and there was 1 case (5\%) with infection. All tumors in the study were histologically confirmed astrocytomas: grade III in $18(82 \%)$ of cases; and grade IV in 4 (19\%). Radiation therapy was carried out in 19 (85\%) of cases, and chemotherapy in only 3 cases (14\%).

No patient is alive at the time of this report. Three (14\%) children died within a month. The longest survival was 22 months with an overall survival rate of 10.5 months. Factors to emerge from this analysis will be discussed and a comparison with other series presented.

\section{5.}

\section{Tyrosinemia and Abnormalities in Pyridoxine (B6) Metabolism}

\section{S.S. SESHIA, T.L. PERRY, K. DAKSHINAMURTI,} Winnipeg, Vancouver

The symptomatology in hereditary tyrosinemia is dominated by hepatic dysfunction and intractable seizures have not been reported in the disorder; the latter occur with disturbances of $B_{6}$ metabolism. We report on a case who had biochemical features of both disorders, an association that has not been previously recognized. R.W., was first hospitalized with right focal motor status epilepticus at 10 months of age; the seizures were only partially controlled. She was rehospitalized in status epilepticus at 20 months; the seizures were generalized tonic clonic, multifocal and myoclonic and did not respond to anticonvulsant drugs or to Pyridoxine. Initially comatose, she later became vegetative and developed dyskinesia. Hepatosplenomegaly and icterus occurred at 22 months and then resolved; investigations for structural, vascular, lysosomal diseases of the nervous system failed to reveal abnormality. EEGs were diffusely abnormal.

Pyridoxal phosphate (PLP) were undetectable in plasma and extremely low in erythrocytes at 21 months of age and only rose slightly after I.V Pyridoxine and pyridoxal. Plasma and CSF examined at 24 months showed marked elevations of Glutamine, Methionine and Tyrosine; CSF levels of homocarnosine and GABA were low. The child died at 25 months; the liver was small and cirrhotic; there was unusual vascularity of the superior colliculi and hypoxic change in the brain. Biochemical studies of the brain showed marked elevations of Glutamine, Methionine and Tyrosine. Homocarnosine was low and GABA reduced in occipital and cerebellar cortices. Activity of P-hydroxyphenylpyruvate hydroxylase an enzyme in the degradation of Tyrosine was undetectable in liver and fibroblasts, suggesting that the abnormalities of Methionine and Tyrosine were, therefore, not caused by hepatic dysfunction but resulted from an enzymatic block. An additional disturbance of $\mathrm{B}_{6}$ metabolism may explain the low levels of plasma/erythrocytic PLP, and of CSF/brain Homocarnosine and GABA. Our case suggests that attention to biochemical abnormalities in CSF and brain may help in defining unusual disorders of the nervous system in children.

46.

\section{Neurochemical alterations in the Basal Ganglia in the Lesch-Nyhan Syndrome}

\section{K.G. LLOYD, M. SHIBUYA, H. HIDAKA, O. HORNYKIEWICZ, Bagneux, TSU, Vienna}

The Lesch-Nyhan (LN) syndrome is an X-linked disease characterized by over-production of purines, and a CNS disorder consisting of mental retardation, spasticity, choreoathetosis, and a compulsive form of selfmutilation. Recently, we described in the basal ganglia of LN patients a reduction of biochemical indices of dopamine (DA) and acetylcholine (ACh) neuron function (Lloyd et al., New Engl. J. Med. 305, 1106, 1981). We have now studied in the caudate nucleus of $3 \mathrm{LN}$ patients (post-mortem 
material) the activity of the phosphodiesterase (PDE) which metabolizes the cyclic nucleotides cAMP and cGMP as well as the concentration of calmodulin $(\mathrm{CaM})$, a potent endogenous activator of PDE. The following results were obtained: $C A M P$-metabolizing $P D E$ (in pmoles $/ \mathrm{min} / \mathrm{mg}$ protein): control - $363 \pm 62$; LN - $215 \pm 31$. cGMP-metabolizing PDE: control - $286 \pm 47 ; \mathrm{LN}-191 \pm 27$. CaM (in units/mg protein): control - 313 $\pm 17 ; \mathrm{LN}-185 \pm 18$. These results show that in the $\mathrm{LN}$ caudate nucleus there is a significant reduction of the $\mathrm{CaM}$ level as well as a decrease of the cAMP- and cGMP-metabolizing PDE activities. Since reduction of PDE activity will result in accumulation of increased amounts of intracellular cAMP and cGMP, this alteration can be expected to potentiate, in the LN striatum, the biological actions of synaptically released DA and ACh respectively. Some pathophysiological implications of these alterations for the CNS symptomatology of LN syndrome will be discussed.

\section{7.}

\section{Therapeutic Transfemoral and Intraoperative Embolization of 54 Large Arteriovenous Malformations of the Brain with Isobutyl-2-Cyanoacrylate}

F. VINUELA, G. DEBRUN, A.J. FOX, C.G. DRAKE, S.J. PEERLESS, J.P. GIR VIN, London, Boston

Analysis of Technical Approach, Morphological Results and Post Embolisation Clinical Outcome

The development of intracranial intravascular navigation with microcatheters and calibrated leak balloons opens a new alternative treatment of intracranial arteriovenous malformations (AVMs).

This technique is particularly useful in high flow AVMs with large single or multiple arterial feeders. If the AVM nidus is unreachable from the transfemoral route, intraoperative embolization is the procedure of choice.

The aim of isobutyl-2-cyanoacrylate (IBC) embolization is obliteration of the AVM nidus. It has been repeatedly shown that proximal occlusion of the AVM feeders by surgical or neuroradiological means is not only useless but disadvantageous in that it removes the chance for proper embolization in the future.

The technical details of transfemoral embolization, intraoperative embolization, combined embolization, without and with surgical removal, will be analyzed. The post embolization morphological and clinical results of right hemisphere, left hemisphere and posterior fossa AVMs will be discussed separately.

Several examples of intraoperative embolization of left dominant hemisphere AVMs involving the rolandic and speech areas will be shown.

In several cases the reduction in size of the AVM nidus by embolization facilitated post embolization surgical removal.

\section{8.}

\section{Intraoperative Embolization (IBC) of Cerebral} Arteriovenous Malformations in the Awake Patient

\section{J.P. GIR VIN, A.J. FOX, F. VINUELA, C.G. DRAKE, London}

There are cases of AVMs in which we believe the safest method of treatment consists of, in the awake patient, direct surgical cannulation of cerebral vessels feeding the arteriovenous malformations and subsequent injection of Isobutyl-2-cyanoacrylate (IBC). The circumstances dictating this choice of treatment include the following: 1) inability to navigate a calibrated leak balloon catheter close enough to the AVM via the femoral arterial approach in the angiographic suite; 2) multiple arterial feeders of such a nature that all of the important feeders could not be reached by balloon catheter; or 3) instances where the AVM lies partially, or totally, within important functional cortical areas, e.g. speech, sensorimotor.

In these cases we advocate the direct intraoperative embolization of the AVM under local anesthesia in the awake patient. Our procedure consists of the following sequence: 1) exposure of the AVM under local anesthesia; 2) cortical mapping of eloquent functional areas by electrical stimulation; 3) identification, isolation, and temporary occlusion (clips) of the feeding vessels during which functional testing is carried out; 4 ) in the absence of any functional impairment, sequential cannulation, angiography and embolization of the multiple feeders by IBC while monitoring the appropriate function; and finally 5) decision as to whether or not surgical resection should be carried out as part of the treatment regime.

This approach has been used in five cases for embolization of cerebral AVMs in such cases. The features of this approach, a summary of results, the advantages, and a description of the problems will be presented.

49.

\section{Cryptic Cerebrovascular Malformations}

\section{H. COHEN, W.S. TUCKER, R.P. HUMPHREYS, R.G. PERRIN,} J.M. BILBAO, Toronto

True cryptic vascular malformations of the brain are defined as those not visualized by appropriate cerebral angiography. The clinical presentation is occasionally seizures, but more often intracerebral hemorrhage, frequently in unusual locations, where surgical access is difficult or impossible. The patients are often young. Firm confirmation can only be made by histologic examination of surgical or post-mortem material. The careful pathological examination of all available material may permit diagnosis, even when the lesion is not seen at microsurgical operation.

Since 1977 , the authors have operated on 8 patients and confirmed cryptic vascular malformations. There were 5 males and 3 females. The average age was 20 years, but 6 of the patients were under that age. Five patients presented with hemorrhage, and 3 patients presented with seizures, but even in the seizure patients, 2 showed evidence of hemorrhage at operation. In several cases there was clinical and radiological evidence of repeated episodes of bleeding. The lesions were all localized by C.T. scan. Appropriate angiography was negative in each case, apart from demonstrating an avascular mass effect after hemorrhage. There were 5 lesions in the cerebral hemispheres, one in the thalamus, one in the brachium pontis, and one in the medulla. Histologic examination of the surgical specimens revealed cavernous hemangioma in 4 , arteriovenous malformation in 2 , and venous angioma in 2 . Only one patient has rebled after surgery, nearly 3 years later, and all patients are living.

\section{0.}

\section{Doppler Correlation of Cervical Bruits}

\section{J.G. D'ALTON, J.W. NORRIS, Toronto}

The clinical significance of the type and location of neck bruits and their correlation with underlying arterial disease is uncertain. During the course of a follow-up study of patients with asymptomatic carotid bruits referred to a carotid doppler laboratory the correlation of neck bruits to the site of the arterial lesion was investigated.

100 consecutively referred patients with 136 neck bruits were divided into four groups according to the site of maximal intensity of the bruit. In the first group, 68 bruits were localized to the angle of the jaw and the majority $(68 \%)$ of these were due to internal carotid artery (ICA) lesions. A further $20 \%$ were due to external carotid artery (ECA) lesions. The second group ( 22 bruits) were localized to the mid cervical region and only $36 \%$ were solely of ICA origin, the remainder being associated with different combinations of arterial lesions. Thirdly, 36 supraclavicular bruits were associated mainly (75\%) with subclavian arterial lesions, but $25 \%$ of this group had unsuspected ICA stenoses. Fourthly, in 10 bruits conducted from the heart, $4(40 \%)$ had associated ICA lesions. Clinical analysis of bruit characteristics showed that tight ICA stenoses $(>75 \%)$ were usually associated with localization to the angle of the jaw and the bruit was high pitched. Intensity and duration of the bruit was less specific.

These findings confirm the clinical impression that high-pitched bruits heard maximally at the angle of the jaw are usually associated with tight ICA stenosis. However, subclavian bruits or cardiac murmurs conducted to this region may be associated with unsuspected underlying ICA disease Carotid doppler ultrasound represents a simple, non-invasive and accurate method of distinguishing this. 
51.

\section{Stroke Probability and Long Term Survival in Post- Endarterectomy Carotid Patients}

H.W.K. BARR, London

A nonrandomized series of 331 patients selected for carotid endarterectomy has been critically reviewed. Pre-existing symptoms, associated risk factors and neurological deficits compare favourably with the patient population in the medically treated Canadian Co-operative Study.

The investigative and operative risks are documented with a mortality rate of $0.5 \%$ and morbidity rate, all mild, of $3.5 \%$. Only $1 \%$ morbidity occurred during the actual operative procedure.

Detailed follow-up was possible in 277 patients, involving a period of 4 months to 14 years.

The incidence of ischemic events in the territory of the post-operative artery, for T.I.A. and stroke, was $6.8 \%$ and $3.2 \%$ respectively, over the total length of follow-up. Of considerable interest is the incidence of T.I.A. and stroke in the previously asymptomatic carotid artery territory, $3.9 \%$ and $3.2 \%$ respectively. The endarterectomy procedure resulted in a reduction in the expected stroke rate of $14.5 \%$ in 2 years to $0.7 \%$. No patients developed a stroke in the operative artery territory in these first two years.

In comparison with the Canadian Co-operative Study, as a guide to natural history, the death rate in the follow-up period between the two groups was identical. However, the combined cumulative stroke and death rate in the surgical group was improved by $50 \%$ over the medical trial patients, indicating a substantial reduction in the anticipated stroke rate.

\section{2.}

\section{The External Carotid Artery in Cerebral Ischemia: Surgical Indications}

\section{G.R. SUTHERLAND, H.J.M. BARNETT, G.K. KIM, S.J. PEERLESS, London, Seoul}

Ischemia in the territory of a known occluded internal carotid artery (ICA) has conventionally been attributed to hemodynamic impairment of perfusion in the territory of the occluded vessel. Athero-platelet thrombi originating from the ICA stump, common carotid artery (CCA) or external carotid artery (ECA) and carried by collaterals to the brain is an important alternate pathogenic mechanism to be considered.

Twenty-three patients, 19 male and 4 female (average age 59 years) presenting with ICA occlusion and transient ischemic attacks (TIAs) with or without stroke are reviewed.

Twelve patients presented with TIAs, 10 with TIAs and stroke and, 1 with stroke only. Cerebral angiography demonstrated an ipsilateral ICA stump in 9, associated common and/or ECA stenosis with an ICA stump in 10 and common and/or ECA stenosis without a stump in 4. Significant contralateral carotid bifurcation atherosclerosis was present in 13 and vertebral basilar disease in 9 .

Surgical treatment consisted of ipsilateral common and external carotid endarterectomy (10 patients) with patchgraft angioplasty (13 patients) and when present obliteration of the ICA stump (19 patients). Six patients were then randomized to the EC/IC Study with atheroma in 20 patients and uncomplicated in 3. Contralateral carotid endarterectomy was performed in 3 patients and EC/IC in 1 . On follow-up 1 to 18 months (mean 5 months), we found resolution of TIAs in 16 , with 2 of the remaining 5 progressing to stroke.

In selected cases, common and external carotid endarterectomy with isolation of the ICA stump removes a emboli from the cerebral circulation. Patchgraft angioplasty optimizes the flow at the carotid bifurcation thus improving existing collaterals and may decrease the rate of progressive atherosclerosis. When extracranial to intracranial anastomosis is contemplated, consideration should first be given to endarterectomy and patchgraft angioplasty.
53.

\section{Clinical Angiographic Correlation of Non-Invasive rCBF Measurements in Suspected Brain Ischemia}

\author{
I.A. AWAD, J.R. LITTLE, A.J. FURLAN, M.A. WEINSTEIN, \\ Cleveland
}

The relation of rCBF measurements to clinical and angiographic data in brain ischemia remains unclear. In this study, 100 patients referred for suspected brain ischemia underwent 4-vessel angiography and noninvasive rCBF measurements as part of their initial evaluation. The $\mathrm{rCBF}$ measurements were accomplished using the Xenon-133 inhalation technique with 16 extracranial detectors. A fast compartment flow rate $(\mathrm{Fg})$, and the newer Initial Slope Index (ISI) were computed for each detector and for each hemisphere. Clinical presentation, angiographic findings, and rCBF data were then categorized and cross-examined for significant correlations.

Forty-eight per cent of patients with hemispheric TIA's and $12 \%$ of patients with completed stroke had a normal rCBF pattern. The most common rCBF pattern in TIA patients was the normal profile, while most stroke patients showed bilateral diffuse abnormalities. Patients with recent stroke ( $<3$ weeks) were more likely to show abnormal rCBF patterns, and diffuse bilateral rCBF abnormalities than patients with old stroke.

Patients with internal carotid artery occlusion most commonly showed diffuse $\mathrm{rCBF}$ abnormalities ( $50 \%$ of patients in the unilateral and bilateral occlusion groups). Furthermore, $12 \%$ of those with bilateral carotid occlusion, and $28 \%$ of those with unilateral carotid occlusion showed normal rCBF patterns. In contrast, the focal rCBF abnormality was the most common rCBF pattern in patients with cerebral artery occlusion $(54 \%$ of cases). Among patients with internal carotid artery stenosis, those with severe arterial narrowing were more likely to show abnormal rCBF patterns, and diffuse bilateral rCBF abnormalities, than patients with mild and moderate narrowing.

Only 2 of the 100 patients had abnormal rCBF patterns and normal angiograms ( $2 \%$ false positive rate). Furthermore, in all cases where angiography and clinical data conflicted, $\mathrm{rCBF}$ accurately reflected the clinical presentation. Overall, ISI proved to be a more sensitive indicator of brain ischemia than Fg.

We conclude that noninvasive $\mathrm{rCBF}$ measurements 1) correlate well with the clinical and angiographic presentation 2) contribute information not available from clinical and angiographic data alone, and therefore 3 ) are valuable to the neurosurgeon evaluating patients with brain ischemia.

\section{4.}

\section{Beta-Blockade Benefits Patients after a Subarachnoid Haemorrhage}

\section{G. NEIL-DWYER, P. WALTERS, J. CRUICKSHANK,} London, England.

The study was set up to assess the effects of adrenergic blockade on the morbidity and mortality following a subarachnoid haemorrhage. All patients in the trial were admitted into the Neurosurgical Unit within 48 hours of a subarachnoid haemorrhage confirmed by lumbar puncture. The patients were aged between 15 and 65 . Patients with a history of cardiovascular or pulmonary disease were omitted. 204 patients entered the randomised, double-blind, between patient study. The first 104 patients were on both Phentolamine ( -blocker) $20 \mathrm{mg} 3$ hourly and Propranolol (B-blocker) $80 \mathrm{mg} 8$ hourly or placebo, the last 100 patients were on either Propranolol or placebo. The first 150 patients were randomised in pairs by computer. The last 50 patients were randomised so that 2 out of every 3 received Propranolol as part of an additional study examining the effects of surgery.

There were 11 patients in the treated and 93 in the control group. The outcome at 28 days was significantly better in the treated group, $\mathrm{P}=$ .0036 . There were significantly fewer deaths in the treated group (13) compared with the control group (21), $\mathrm{P}=.026$. Patients reviewed at 1 year showed greater improvement in the treated group. The results will be discussed with particular reference to aneurysms, surgery and the possible reasons for the beneficial effects of Propranolol. 
55.

\section{Type III Collagen Deficiency and Ruptured Cerebral Aneurysms}

G. NEIL-DWYER, J. BARTLETT, A. DOSHI, F.M. POPE, A.C. NICHOLLS, P. NARCISI, London, England

As inherited type III collagen deficiency causes arterial fragility and aneurysms in Ehlers Donlos Syndrome Type IV and since $70 \%$ of arterial walls are type III collagen we decided to study the collagen patterns in patients with ruptured cerebral aneurysms.

Skin and temporal arterial biopsies were obtained from 17 patients un dergoing surgery for ruptured cerebral aneurysms. Control specimens were taken from 6 age and sex matched controls, 3 of whom had gliomas and 3 meningiomas. Some skin and arterial samples were frozen unfixed for biochemical examination of their collagens whilst others were placed in Dulbeccos medium for primary fibroblast culture. Collagens synthesized by these cells were labelled in serum free medium with $1 \mu \mathrm{C} / \mathrm{ml}$ of $\mathrm{U}^{14} \mathrm{C}$ glycine and $U^{14}$ proline in the presence of ascorbate and $B$-amino proprionitrite. After limited pepsin digestion of tissue or radioactively labelled collagens, type III/I collagen ratios were examined either by interrupted polyacrylamide gel electrophoresis using trisborate buffers or by carboxymethyl cellulose chromatography after heat denaturation.

All the control group had normal collagen ratios while 11 of the 17 aneurysm patients studies showed type III collagen deficiency.

These results will be discussed in the light of the congenital and acquired theories on the aetiology of cerebral aneurysms. The effects of age, sex, family history, angiographic anomalies and hypertension will be considered.

\section{6.}

\section{Serial Measurements of Cerebral Blood Flow (CBF) as a Guide to Surgery in Patients with Ruptured Intracranial Aneurysms}

\section{G.G. FERGUSON, J.K. FARRAR, K. MEGURO, C.G. DRAKE,} S.J. PEERLESS, London

We have measured $\mathrm{CBF}$ in 55 patients with ruptured intracranial aneurysms during the course of their illness to study the progressive changes in flow in individual patients in relation to the initial CT scan findings, changing clinical grade, the development of arterial vasospasm, cerebral infarction, and surgery.

We found a significant depression of mean hemispheric flow in all patients with $\mathrm{SAH}$ - the magnitude varying with clinical grade, but showing a tendency to recovery with time. In some patients, flows were unexpectedly low, invariably the result of severe spasm - "the silent spasm syndrome". In others, CBF was unexpectedly high, the result of focal deficits related to cerebral infarction. There was good correlation between hemispheric flow and the degree of angiographic spasm, this being especially the case for spasm in the middle cerebral artery territory. Surgery commonly depressed flows.

Thirty-one patients had CT scans within 7 days of the initial hemorrhage. Of the patients with blood in the basal cisterns, $82 \%$ developed moderate to severe spasm, and this group had major instability of cerebral hemodynamics. They developed severe depressions of flow particularly during the second post-SAH week, and had a poor clinical outcome.

We have found that mean hemispheric CBF values determined by the inhalation technique are a useful physiological index in evaluating patients with SAH. Such measurements may be used as a guide to the timing of surgery, in addition to the more traditional guides of the patient's clinical state and the radiological appearance of the cerebral arteries. Patients with low flows $(<35 \mathrm{ml} / 100 \mathrm{~g} / \mathrm{min})$ have limited cerebrovascular reserve and are at higher risk with surgery and intraoperative hypotension. Surgery in such patients should probably be delayed if possible until flows have recovered. In patients with near normal flows, surgery does not carry undue hemodynamic risk.

\section{7.}

\section{The Distribution of Intracranial Branch Occlusions}

\section{G. GACS, A. FOX, H.J.M. BARNETT, London}

Knowledge of the pathogenesis of occlusions of the intracranial arteries would be important to therapy. The embolic theory has been favoured recently, but primary evidence of it is still lacking.

Considerable, regular differences have been found in the incidence of occlusions among the individual intracranial arteries on cerebral angiograms. Among 221 intracranial occlusions observed angiographically at 1:14 ratio was found between the anterior and middle cerebral arteries. The most frequently affected cortical branches are the angular and the parietal branches of the middle cerebral artery.

The authors examined the spontaneous pathways of balloon-catheters used for superselective angiography drifting freely in the blood stream. A striking similarity has been found between the distribution of occlusions in various cerebral arteries and the statistical regularity of the pathways of the balloon.

Based on the assumption that the course of the balloon is determined by the same hydrodynamic laws as that of an embolus of similar size, it is concluded that the majority of occlusions of cerebral arteries are embolic in origin.

The suggestion gains a further confirmation by the same distribution of infarcts found on CT scan in 450 cases.

Other possible causes of the frequency pattern are discussed.

58.

\section{Spontaneous Carotid-Cavernous Fistula: A Dural AVM \\ F. VINUELA, A.J. FOX, G. DEBRUN, C.G. DRAKE, S.J. PEERLESS,} J.P. GIRVIN, London

The clinical presentation and the angiographic appearance of spontaneous carotid-cavernous $(\mathrm{cc})$ fistulas are entirely different from post traumatic ones. From the clinical viewpoint spontaneous cc fistula is more often seen in middle-aged women, it does not have a previous history of trauma, it has a prolonged clinical course and sometimes it may cure spontaneously. Angiographically, it has a dural external carotid/internal carotid blood supply, a vascular "nidus" is identified, and it has relatively slow arteriovenous shunting. This lesion is really a dural AVM, rather than a true fistula.

It also has an entirely different therapeutic approach, when compared with the post traumatic fistula. In most cases, the aim of the interventional procedure is to occlude the myriad abnormal dural vessels using either Ivalon particles or Isobutyl-2-cyanoacrylate via the external carotid artery. Proximal occlusion of the arterial feeders by balloon detachment is useless and disadvantageous when dealing with this type of malformation.

The clinical spectrum observed in nine patients with dural AVMs thought clinically to be spontaneous cc fistulas will be presented. The angiographic characteristics and the therapeutic alternatives will be shown and discussed.

The laboratory work for the development of a latex calibrated leak balloon/teflon catheter system for safe embolization with IBC of this vascular malformation will also be shown. 


\section{Poster Presentations}

P1.

\section{One Hospital's Experience with Thrombotic Thrombocytopenic Purpura}

\section{TSOUKAS, C. KRIEGER, G. BLAKE, Montreal}

Four patients have been seen with throbotic thrombocytopenic purpura in one general hospital over a two year period. All patients presented with the cardinal features of this disease namely hemolytic anemia, thrombocytopenia, fluctuating neurologic signs, fever and renal disease. The neurologic presentation consisted of increasing headaches followed by agitation, progressive obtundation and coma. In these patients no focal findings were present on initial neurologic examination. Cranial CT scans were often unremarkable. All patients had a rapid downhill course with 2 dying with 3 days of admission. The treatment of the 2 surviving patients included antiplatelet agents and vincristine. The rationale for this treatment will be discussed.

\section{P2.}

\section{The Effect of Pairing Scanning Training with Block Design Training in the Rehabilitation of Left Hemi-Inattention}

\section{COLLINS, G. YOUNG, M. HREN, Toronto}

Twenty-seven stroke patients with left hemi-inattention (based on their performance on a number cancellation task) were divided into 3 groups of 9 , controlling for degree of left neglect, age and time since onset of stroke. Every patient was pretested on measures of psychometric intelligence, visual-spatial organization, reading, writing, face-counting, bisecting a line, letter cancellation and activities of daily living. Every patient then received 20 successive days of training with an occupational therapist. Every day, group I received 20 minutes with a visual scanning machine, 20 minutes on cancellation tasks and 20 minutes on block design. Group II received 20 minutes with the scanning machine, 20 minutes on cancellation tasks and 20 minutes of routine occupational therapy. Group III, the control group, received one hour of routine occupational therapy. Every patient was then retested on the pre-test measures, by one of the experimenters who had no idea of the composition of groups.

In contrast to previous findings, the groups did not differ significantly on measures of psychometric intelligence. However, scanning behaviour and visual-spatial organization improved significantly in the two experimental groups and clear linear trends were present in the cases of reading, writing and facecounting. These results support the previous findings that scanning and block design training are effective treatment methods in the rehabilitation of left hemi-inattention and related problems and furthermore, that when these methods are paired, the effects are significantly greater than when they are used independently.

\section{P3.}

\section{The Syndrome of Bilateral Paramedian Thalamic Infraction}

\section{A. GUBERMAN, D. STUSS, Ottawa}

The paramedian thalamic branches arising from the posterior cerebral arteries near the basilar bifurcation occasionally supply both mesial thalami via a common trunk arising from one side. We have done extensive neurological and neuropsychological investigations in the acute and chronic phases in 2 patients with infarction in this territory demonstrated on CT-scan.

Both cases were middle-aged previously healthy males with mild hypertension, who presented with transient coma. In each case, severe amnesia, apathy and vertical gaze abnormalities were present upon awakening. Case 1 also demonstrated transient asterixis, diminished facial expression, hypersomnia and a full-blown Korsakoff's syndrome. His CTscan initially showed bilateral mesial thalamic enhancing lesions replaced by low-density areas on follow-up. Case 2 showed a low-density lesion in the right mesial thalamus on follow-up. Ten months later case 2 revealed only a minimal memory deficit but 1 year after onset case 1 showed a persistent severe amnesia and moderate dementia.

This syndrome, with its virtually diagnostic combination of signs must be considered in the differential diagnosis of coma and sudden onset of amnesia. These cases illustrate the importance of mesial thalamic areas, including the dorsal median nuclei, in memory.

P4.

\section{Risks of Cerebral Angiography in Cerebrovascular Disease}

\section{M.D. CUSIMANO, J.G. D'ALTON, P.W. COOPER, E.E. KASSEL,} J.W. NORRIS, Toronto

Considerations of risks of cerebral angiography is essential in strategy for management of patients with cerebrovascular disease. Recent progress in technique and equipment have lead to increased accuracy and potential safety of the procedure. This study was performed to compare the risks in a cerebrovascular population compared to non-cerebrovascular patients.

Records of 538 consecutive patients who had 653 cerebral angriograms performed by the femoral route were reviewed for complications attributable to angiography. There were 273 patients with cerebrovascular (C.V.) disease and 265 others, having 307 and 346 angiograms respectively. The mean age in the C.V. group was 59 and $8(2.6 \%)$ suffered a complication, of which 6 were neurological, 2 being permanent. One patient died (mortality $0.3 \%$ ). In the non C.V. group, the mean age was 48 and there were 3 complications, all of which were transient neurological, with no residual deficit.

While there was no statistical difference in the incidence of complications between the two groups, the only permanent complications occurred in the cerebrovascular population.

\section{P5.}

\section{Treatment of Focal Cerebral Ischemia with Prostacyclin and Indomethacin}

\section{I.A. AWAD, J.R. LITTLE, V. SKRINSKA, F. LUCAS, Cleveland}

The object of this investigation was to study the effects of Prostacyclin $\left(\mathrm{PGI}_{2}\right)$, with and without Indomethacin, upon the evolution of ischemic edema and cerebral infarction. Thirty-five fasted adult cats lightly anesthetized with nitrous oxide underwent right middle cerebral artery (MCA) occlusion. Eleven cats received an intra-carotid infusion of $\mathrm{PGI}_{2}$ in buffered saline $\mathrm{pH} 10.2(100 \mathrm{ng} / \mathrm{kg} / \mathrm{min}$ at $0.01 \mathrm{ml} / \mathrm{kg} / \mathrm{min}), 10$ cats received the same infusion plus a single dose of intravenous Indomethacin $4 \mathrm{mg} / \mathrm{kg}$, and 11 cats received intracarotid buffered saline $\mathrm{pH} 10.2(0.01 \mathrm{ml} / \mathrm{kg} / \mathrm{min})$ without therapeutic agents. Treatment with $\mathrm{PGI}_{2}$ was started upon $\mathrm{MCA}$ occlusion and continued for 6 hours, where as Indomethacin was given immediately prior to occlusion. Thirty minutes prior to perfusion, the animals were given fluorescein and Evans blue by intravenous injection. The cats were perfused-fixed in vivo with carbon and buffered formalin 6 hours after MCA occlusion. Another 3 cats received tritium labeled $\mathrm{PGI}_{2}$, and peripheral venous samples were collected and assayed for $\mathrm{PGI}_{2}$ and its alpha keto metabolite.

Mean arterial pressure was stable in treated animals during 6 hours of MCA occlusion, while untreated cats had significant $(\alpha=0.05)$ progressive hypertension during that period. The rCBF (measured by the intracarotid ${ }^{133} \mathrm{Xe}$ method) decreased markedly in all animals immediately upon MCA occlusion. Untreated animals had a significant progressive improvement in $\mathrm{rCBF}$ during the occlusion period $(\alpha=0.005)$, while treated animals had no such improvement. Quantitative EEG changes, gross edema, areas of fluorescein extravasation and microscopic morphology (edema and infarct size) were not significantly different in the three groups. $\mathrm{PGI}_{2}$ prevented the extravasation of Evans blue dye observed in all un- 
treated animals. Systemic $\mathbf{P G I}_{2}$ levels were significant despite intracarotid administration.

We conclude that 1 ) intra-carotid $\mathrm{PGI}_{2}$ has a protective effect against the breakdown of the blood-brain barrier to protein bound dyes seen in ischemic edema; 2) the systemic hemodynamic effects of $\mathrm{PGI}_{2}$ in the presence of impaired autoregulation, may compromise $\mathrm{rCBF}$ in the ischemic zone and offset any direct beneficial effects; and 3) Indomethacin failed to modify the effects of $\mathrm{PGI}_{2}$.

\section{P6.}

\section{Effect of Tissue Heterogeneity on Cerebral Blood Flow Measurement with the Equilibrium Method Using Inhaled 0-15 Carbon Dioxide}

\section{P. HERSCOVITCH, M.E. RAICHLE, St. Louis}

The equilibrium $0-15 \mathrm{CO} 2$ inhalation method for measuring regional cerebral blood flow (rCBF) with positron emission tomography (PET) is based on a one compartment model with both homogeneous flow and partition coefficient $(\gamma)$ for water. However, given the limited spatial resolution of PET, a region of interest (ROI) will contain a mixture of grey matter (GM) and white matter (WM). A computer model was used to examine the effect of this heterogeneity on rCBF measurement.

With GM and WM flows of 80 and $20 \mathrm{ml} / \mathrm{min} / \mathrm{hg}$ respectively, and model of $1.0 \mathrm{ml} / \mathrm{g}, \mathrm{rCBF}$ was underestimated by a maximum of $23 \%$ in a $30 \% \mathrm{GM}$ ROI, and by $8 \%$ in an $80 \%$ GM ROI. The calculation of rCBF was very sensitive to choice of model

Simulation of stepwise changes in GM flow resulted in differing changes in measured flow due to the model's non-linear relationship between rCBF and tissue radioactivity. Increasing WM $\gamma$ as in edema improved flow measurement accuracy in predominantly WM ROI. Varying GM $\gamma$ from 0.9 to 1.05 to simulate brain tumor lead to maximum flow errors of $32 \%$.

Thus, in both normal and pathological states, large errors in rCBF measurement can occur with the equilibrium method due to tissue heterogeneity in the ROI.

\section{R7.}

\section{Cerebrospinal Fluid Shunts: A Dynamic Evaluation of a Hemodynamic Problem using "Intra-Shunt" Pressure Monitoring and Infusion Studies}

\section{C.E.U. EKONG, D.W. ROWED, Toronto}

The physical patency of a cerebrospinal fluid (CSF) shunt does not guarantee optimal functional competence. Yet many radiographic and clinical procedures for assessing shunt function are aimed at physical patency alone. A shunt may be patent but not reducing intraventricular pressure adequately for various reasons including the use of a valve with too high an opening pressure, partial block age of the valve, or the presence of a reduced surface area for absorption of CSF as in a peritoneal cyst at the end of the shunt.

Patients with clinical evidence of shunt block age were assessed with CT scans, radioisotope shunt-o-gram, intra-shunt pressure (ISP) monitoring and infusion studies. The ISP monitoring was done through a 25 gauge needle inserted into the shunt reservoir under sterile conditions and an overnight continuous pressure recording was performed. This was followed by infusion of Elliot's solution at the rates of $0.7 \mathrm{cc} / \mathrm{min} ., 1.4 \mathrm{cc} / \mathrm{min}$. and $3.0 \mathrm{cc} / \mathrm{min}$. respectively while the pressure was recorded. The baseline ISP was over 10 Torr in all the patients and showed frequent B waves. Infusion with Elliot's solution resulted in marked elevation of the pressure and failure to return to the baseline pressure 5 minutes after infusion. Following successful revision of the shunt, the baseline recording became the opening pressure of the valve, the B waves became very few, and the ISP returned to the opening pressure of the valve within 5 minutes of infusion with Elliot's solution. Our results indicate that intra-shunt pressure monitoring and infusion studies may be useful techniques for assessing the functional status of CSF shunts.
P8.

\section{Surgical Management of the Neck-Tongue Syndrome - Case Report}

\section{K. ELISEVICH, J. STRATFORD, G. BRAY, Montreal}

Unilateral numbness of the tongue associated with ipsilateral neck or occipital pain on head turning was described by Lance and Anthony ( $J$. Neurol. Neurosurg. Psychiatry $1980 ; 43,97)$ and elaborated by Bogduk (J. Neurol. Neurosurg. Psychiatry $1981 ; 44,202$ ). The syndrome is attributed to abnormal subluxation of the lateral atlantoaxial joint with compression of the $\mathrm{C} 2$ ventral ramus. The sensory symptoms are presumably caused by the involvement of afferent fibres from the lingual nerve that pass via the hypoglossal nerve to the $\mathrm{C} 2$ ventral ramus.

Our patient is a 53 year old woman with a seven year history of unilateral neck discomfort, which was initially aggravated by head turning, and a one year history of ipsilateral tingling in the tongue. Radiologic assessment demonstrated assimilation of the posterior arch of the atlas to the occipital bone and a normal myelogram. After operative resection of a fibrosed $\mathrm{C} 2$ ventral ramus she experienced a diminution of pain and a marked reduction in the severity and frequency of paresthesias in the tongue.

P9.

\section{Intraventricular Morphine for Intractable Pain}

\section{H. HUGENHOLTZ, M.T. RICHARD, W. SO, Ottawa}

Potent analgesia follows intrathecal administration of morphine in rats (Yaksh T.L. and Rudy T.A., 1976). Its successful application in patients was first reported by Wang J.K. et al (1979).

Chronic administration of morphine can be directed into the spinal subarachnoid space or the ventricular system using an Ommaya reservoir (Leavens M.E. et al, 1982) or a constant infusion pump (Onofrio B.M. et al, 1981).

This report details the methodology, dosage, response and side effects of intraventricular morphine over 28 days in a 36 year old man with intractable retrosternal chest pain secondary to terminal bronchogenic carcinoma until his death from additional pulmonary emboli. Predictably, he developed tolerance to the intrathecal morphine, and required increasing doses at decreasing intervals, although pain relief remained satisfactory.

Despite the administration of up to $10 \mathrm{mg}$ of morphine into his lateral ventricle within 24 hours, reported hazardous complications such as cardiovascular, respiratory and neurologic depression were not observed. The significance of these observations is discussed.

P10.

\section{Morphometric and Anatomical Correlation of Dense Microsphere and Senile Plaque Formation in Alzheimer's Disease}

\section{P. AVERBACK, Montreal}

Dense microspheres (DMS) are rare parasynaptic intraneuronal structures consisting of membrane bound protein accumulations found principally in cerebral cortex, hypothalamus, amygdala and hippocampus of post-infancy normal human brain (Averback, Arch. Pathol. Lab. Med., in press, 1981, Fed. Proc., in press, 1982). DMS relevance to the senile plaque (SP) of Alzheimer's disease (AD) is based on neurohistological, electron microscopic, and quantitative morphological evidence from analyses of postmortem human brain $(\mathrm{AD}, \mathrm{N}=42$, mean age $=74.3$, normal control (NC) $\mathrm{N}=120$, mean age $=60.4$ ), which indicate: 1) precise and exclusive identity of cytological and histological location of DMS and SP; 2) virtually identical neuroanatomical topography of DMS and SP; 3) the DMS is the only presently known non-perikaryal normal neuronal CNS histological structure whose frequency per unit volume is of similar order of magnitude as the SP; 4) the DMS is the only presently known distinguishable non-perikaryal CNS histological structure whose disappearance approximately coincides in time and magnitude with SP ap- 
pearance; and 5) tissue spatial distribution (D ratio) of DMS and SP are nearly identical, and these are the only two presently known randomly distributed structures in the appropriate brain nuclei at their order of magnitude of tissue density.

NC Nv(DMS) $581 / \mathrm{mm}^{3}$ (s.d. 407) D ratio DMS .950

AD Nv (DMS) $222 / \mathrm{mm}^{3}$ (s.d. 103) (p<.01) D ratio SP .915

AD Vv (DMS + DDMS + SP) $574 / \mathrm{mm}^{3}$ (s.d. 272)

Mean DMS volume in the hippocampus in senility is $65.5 \mathrm{u}^{3}$, compared to $28.5 \mathrm{u}^{3}$ in temporal neocortex; mean SP diameters in these areas are $61.3 \mathrm{u}^{3}$ and $34.0 \mathrm{u}^{3}$ respectively, a nearly linear proportion $(\mathrm{p}<.001)$ in which the distributions show negligible overlap. The potential clinical relevance of these findings is discussed.

\section{P11.}

\section{The Dense Microsphere: A Newly Delineated Origin of the Senile Plaque in Human Brain}

\section{P. AVERBACK, Montreal}

The dense microsphere (DMS) is a normal human CNS structure acquired in infancy which has significant anatomical, temporal and quantitative relationships to the senile plaque (SP) of Alzheimer's disease (AD) (Arch. Pathol. Lab. Med., in press, 1981, Fed. Proc., in press, 1982). Bielschowsky, Bodian, Holmes, Congo red, periodic acid Schiff and hematoxylin-eosin stained frozen and paraffin sections from post-mortem samples of $\mathrm{AD}(\mathrm{N}=42$, mean age $=74.3)$ and normal $(\mathrm{N}=120$, mean age $=60.4$ ) cerebral cortex were analyzed and over 1200 photomicrographs were enlarged, rotated, and sequentially collated in the production of plates illustrating the transformation of DMS into SP in human brain. DMS initially become disrupted by small surface fractures or scallopings or by a variety of topological transformations with an intact surface. Subsequently progressive fragmentation, fading, folding and change of size and tinctorial affinity or lysis by stepwise or concurrent fragmentation and dissolution occurs, in the process of which DMS remnants may be traced through the evolution into SP in over $70 \%$ of examples studied. Preliminary isolation and analysis of the DMS is described, and the potential therapeutic and preventive application of these findings to $A D$ is discussed.

\section{P12.}

\section{Neonatal Hyperammonemic Coma and Variable Neurologic Deficits}

\section{H. DARWISH, F. SNYDER, H. SARNAT, H. PARSON, Calgary}

The encephalopathy associated with hepatic disease in the adult or Reye's syndrome in the child correlates poorly with blood ammonia levels. Of the many aminoacids, aspartic acid has been identified as excitatory in the nervous system and a cause of neonatal seizures. Ophthalmoplegia and bulbar palsy have been reported in maple syrup urine disease.

We present 3 neonates who fit the criteria for hyperammonemic coma. In all, ammonia levels were more than 5 times normal, and they had distinctly different neurologic manifestations. We have attempted to correlate the neurologic deficits with their plasma and CSF aminoacid profiles. The first patient had ornithine transcarbamylase deficiency and at 39 hours of age, had fixed, dilated pupils, absent corneal and caloric responses, and hypotonia.

The second patient had propionic acidemia and at 6 days of age was stuporous, had frequent myoclonic jerks and hypertonia. The corneal reflex was absent but she had a prominent startle response to sound stimuli.

The third patient had argininosuccinase deficiency, and was comatose on day 4 , with absent gag reflex and hypertonia.

The EEG in the first patient showed intermittent paroxysms of biphasic spikes and notched very high amplitude delta with a steep gradient. In the other 2 patients the EEG was characterized by very low amplitude fast activity.

These findings correlated with 400,975 , and $450 \mathrm{umol} / \mathrm{L}$ of plasma $\mathrm{NH}_{3}$ in the 1st, 2nd and 3rd patients respectively. Unexplained hypomagnesemia and hypocalcemia were present.

The plasma aminoacids in the first patient were characterized by elevation in glycine and alanine, usually considered inhibitory to the nervous system. The 3 rd patient had elevation of citrulline, and in the 2 nd patient the aminoacid changes were inconsistent. The CSF aminoacids in the 1st patient differed significantly from the 2 nd by showing significant elevation of aspartic acid.

We believe that prospective evaluation of CSF and plasma aminoacids may explain differences in neurologic findings in neonates with "hyperammonemic coma".

P13.

\section{Fontanel Pressure Measurements in Infants}

\section{L.P. IVAN, A. BADEJO, Ottawa}

Fontanel pressure measurements were performed on 101 healthy newborn infants in a neonatal nursery between one to four days of life. The Ladd fiberoptic sensor and pressure monitor were used with three different holding devices which were compared to each other. The mean values were $9.45,8.45$ and $8.2 \mathrm{mmHg}$ with a standard error of $\pm 0.46, \pm 0.37$ and $\pm 0.30 \mathrm{mmHg}$ respectively.

Fontanel pressure measurements were performed on 44 infants comparing two different holding devices. The infants' ages ranged from one week to six months.

The fontanel pressure was compared to epidural, ventricular and lumbar subarachnoid fluid pressure in small groups of infants and good correlation between the epidural, fontanel and lumbar pressure was found, while discrepancies between the ventricular and fontanel pressure were noted.

From this material, we conclude that regardless of the holding device, fontanel pressure measurement with the fiberoptic sensor is a consistent reading and reflects the intracranial pressure correctly. There is a positive close linear correlation between epidural and fontanel pressure, and between spinal subarachnoid and fontanel pressure. There were discrepancies between ventricular fluid pressure and fontanel pressure, the ventricular pressure being always lower.

\section{P14.}

\section{Hemimegalencephaly in Childhood}

\section{G. RONEN, J.A.R. TIBBLES, P.R. CAMFIELD, Halifax}

We have seen 3 infants with hemimegalencephaly at the I.W.K. Hospital for Children. Case (1) had left hemihypertrophy, hemimegalencephaly, cutaneous vascular nevi, varicosity of veins and retinal colobomata. He also had developmental retardation (DQ 50) and epilepsy. CT scan showed a thick left cerebral mantle, dilated ventricle and full subarachnoid space.

Case (2) presented with torticollis and enlargement of the right hemicranium. CT scan showed an enlarged right hemisphere. DQ at 13 months was 95 and she has not developed epilepsy.

Case (3) was less clear-cut. She presented with marked megalencephaly. On CT scan there were dilated ventricles maximal on the right and a wellpreserved cerebral mantle. A VP shunt was performed despite which the head circumference has remained at between 3 and 4 standard deviations above the mean. At age 3 her DQ was 100 , but, she has developed focal epilepsy.

In conclusion, this disorder is mostly sporadic: when associated with angiomata and other malformations it constitutes the Klippel Trenaunay Syndrome. While the literature suggests that mental retardation and epilepsy are associated, diagnosis by CT scan of less severe cases indicates that this is not invariable. The exclusion of hydrocephalus may be difficult, but, the thickness of the cerebral mantle and generous subarachnoid space usually indicate the correct diagnosis.

P15.

\section{Pattern Shift Visual Evoked Responses in Mild Multiple Sclerosis}

W.J. BECKER, A. HARRISON, T.P. SELAND, I. RICHARDS, Calgary

19 patients with mild multiple sclerosis (MS) and minimal disability were recalled for repeat pattern shift visual evoked response (PSVER) studies 4 
to 30 months (mean 19 months) after their first test. To be recalled, patients had to have one eye with normal PSVER latency (normal eye) and one eye with a prolonged PSVER latency (abnormal eye) on the first test.

None of the normal eyes showed a latency reduction of more than 4 msec. over the study period and were similar to the control group.

4 abnormal eyes had a latency reduction of $10 \mathrm{msec}$. or more (range 10 30 ). All 4 eyes had had onset of mild clinical optic neuritis in the 5 weeks prior to the first test.

3 normal eyes and 2 abnormal eyes had latency increases of $10 \mathrm{msec}$. or more (range 13-45). 3 had been symptomatic, 2 asymptomatic in the interval between tests.

The remaining 29 eyes remained stable, with latency changes of $7 \mathrm{msec}$. or less, similar to our control group.

The PSVER can add to our knowledge of the natural history of M.S. New PSVER latency increases, both symptomatic and asymptomatic, may indicate new optic nerve demyelinating lesions.

PSVER latency reductions are common in the months immediately after optic neuritis, and may indicate resolution of edema and or remyelination.

\section{P16.}

\section{The Effects of Age on Human Event-Related Potentials}

T.W. PICTON, S.C. CHAMPAGNE, A.M. CERRI, D.T. STUSS, R.F. NELSON, Ottawa

The detection of an occasional target-stimulus in a train of standard stimuli is associated with a late positive wave (P3) in the human eventrelated potential. In order to obtain baseline data for the possible use of this wave in evaluating disordered cognition, we have examined the effects of age and discrimination-difficulty on the event-related potential following a detected target-stimulus. In the first experiment recordings were made from two male and two female subjects in each decade from 20 to 80 years. Brief auditory stimuli were presented through earphones at a rate of $1 / 1.1 \mathrm{se}$ conds with an intensity of $90 \mathrm{~dB}$ peak SPL. Ten percent of the stimuli were targets $(2000 \mathrm{~Hz})$ and $90 \%$ were standards $(1000 \mathrm{~Hz})$. The subjects were asked to detect and keep a running mental count of the number of targets. There was a significant and regular decrease in the latency of the P3 component with age $(r=0.67 ; p<0.005)$. The regression line for this relationship was: latency $(\mathrm{ms})=1.9 \mathrm{x}$ (age in years) +260 . There was also a significant decrease in amplitude with age $(r=-0.53 ; p<0.01)$. The regression line for this relationship was: amplitude (uV) $=20-0.17 \mathrm{x}$ (age in years). In the second study the standard stimuli had a frequency of 500 $\mathrm{Hz}$ and the targets had frequencies of $600,800,1200$ or $2000 \mathrm{~Hz}$. This paradigm was evaluated in 5 young female subjects (20-40 years) and in 5 old female subjects (60-80 years). The P3-latency was again longer in the older subjects. In both age groups the P3-latency was longer with the more difficult target-discriminations. There was no interaction between these effects, and the P3-latency in the old subjects remained long even when their reaction time and accuracy of performance were equivalent to those in the young subjects. There is, therefore, a significant increase in P3-latency with age that is independent of the difficulty of stimulus-discrimination.

\section{P17.}

\section{Conduction of Sensory Action Potentials Across the Posterior Fossa in Infratentorial Space-Occupying Lesions in Man}

\section{F. GENTILI, A.D. WANG, L. SYMON, Toronto, London, England}

Since the demonstration of evoked electrical activity on scalp recordings following median nerve stimulation by Dawson in 1947, there has been an increasing interest in the use of evoked potentials to evaluate various aspects of brain function. A method of analyzing the somatosensory evoked response that has proved of value has been that of measuring central conduction time, defined as the time interval between the N14 peak representing the arrival of the afferent volley of the dorsal column nuclei and the N20 peak which signals the arrival of the impulse at the somatosensory cortex. Both animal experimentation and clinical studies have suggested that prolongation of this central conduction time may be related to cerebral perfusion.
Central conduction time following median nerve stimulation was recorded in 11 normal healthy volunteers and 28 patients with infratentorial space-occupying lesions. Fourteen $(50 \%)$ of the patients with posterior fossa lesions showed a prolonged central conduction time which involved that segment of the conduction time between the dorsal column nuclei generators and the generation site of P15 which we believed to be in the upper brainstem or thalamus. Three cases showed prolongation of the N14-P15 segment although total central conduction time was within normal limits. Only one case with marked hydrocephalus showed prolongation both before and after the P15 wave. Among the 7 cases of C.P. angle tumours with prolonged central conduction time, 3 showed bilateral changes while 4 cases with unilateral prolonged conduction showed this abnormality on the side of the tumour, contralateral to stimulation. This would suggest therefore that the main impact of an acoustic neuroma on the sensory conduction pathways occurs high up in the angle above the level of the sensory decussation. The pathophysiology of prolongation of central conduction time in these cases and the possible role of somatosensory evoked potentials during surgery will also be discussed.

P 18.

\section{The Use of Auditory Evoked Responses as a Diagnostic Measure in Determining Unilateral Cortical Injury in Childhood}

\section{M.J. TAYLOR, F.V. KHADEM, A. O'GORMAN, G.V. WATTERS, Toronto, Montreal}

It has been shown recently, in adults with confirmed sites of cortical lesions that long latency auditory evoked responses (AERs) reflect the intactness of the temporal-parietal cortex. We compared AERs to standard neurological measures of cortical damage in a series of hemiparetic children. Twenty left and twenty right hemiparetic children were studied; 13 and 11 from each group, respectively, suffered prenatal insult, the remaining 7 and 9 suffered peri- or postnatal insult. The children were selected on the basis of extensive neurological assessment or motor and sensory impairment; only those with exclusively unilateral impairment were included. The children also had a CT scan. Their ages ranged from $6-17$ years, with a mean of 12 years.

The AERs were recorded over the left and right primary auditory cortex to monaural click stimuli presented at $1 / \mathrm{sec}$. At least two averages of 64 trials were recorded from each ear in all children. The AERs were recorded and scored independently of the other assessments and without knowledge of whether the child had suffered left or right hemisphere damage.

The AERs were missing completely or partially over the damaged hemisphere in 29 of the 40 cases, and when recorded bilaterally the AERs were usually both larger amplitude and better defined over the intact hemisphere. In only 5 cases, all with prenatal insult, were the AERs within normal range. High correlations were found between the tests of motor and, to a lesser extent, sensory impairment and the AERs. The AER data was more variable for the left hemisphere damaged children, though, particularly the postnatal group, yielding smaller interhemispheric differences for this group. Overall the AERs from the hemiparetic children were more variable and more poorly defined than those from age-matched controls.

These data were correlated with the CT scans in which the severity of damage was carefully graded, without knowledge of the side of hemiparesis. The CT scans correlated highly with the AER results and the side of damage; only in a few cases were the CT scans completely normal. The normal AERs and CT scans were not found with the same patients. It is suggested that the AERs offer an objective measure of cortical function at the temporal-parietal lobes and in cases such as these could be used in conjunction with the CT scan in assessing damage. The AERs may be particularly appropriate when dealing with functional rather than structural damage.

P19.

\section{Multimodality Evoked Potentials (EPs)}

\section{A.R.M. UPTON, L. McDONALD, T. CERMAK, W. SALTARELLI, Hamilton}

Multimodality evoked potentials have been available as diagnostic 
procedures at McMaster for the last five years. Analysis of 1066 consecutive patients ( 100 diagnoses) over a period of 10 months demonstrated abnormalities in $61.3 \%$ of visual (VEP), $52.7 \%$ of cortical somatosensory (CSSEP), 52.4\% of subcortical somatosensory (SCSSEP) and $46.5 \%$ of auditory (BSAEP) evoked potentials (EPs) (Male 503; age $28.7 \pm 22.9$. Female 563; age $31.6 \pm 20.1$ )

In patients with possible M.S. (185. 17.4\%) there were more females $(61.6 \%)$ with most frequent abnormalities in the VEP (69\%). Abnormalities were seen in other sometimes asymptomatic sensory systems (BSSEP, $65 \%$; CSSEP, $57 \%$; AEP, $55.5 \%$; all abnormal, $26.1 \%$; one abnormality or more, $80 \%$ ).

There was a male preponderence in 85 infants with respiratory problems $(57.6 \% ; 54.1 \%$ abnormal) and in 64 patients with head injuries $(56.3 \%)$. One hundred and seven patients with vertigo ( $59.4 \%$ female) showed a higher percentage of abnormalities in the VEP (48.1\%). Deep brain recordings in 80 patients have helped to establish the origin of components of subcortical, auditory and somatosensory EPs.

Since EPs demonstrate function rather than structure, they supplemented CAT scans in patients with brainstem anoxic, post-traumatic, demyelinating and vascular lesions, Reyes Syndrome, coma and brain death. The results demonstrate that Multimodality recording greatly increases the diagnostic value of any one recording.

\section{P20.}

\section{Single Fibre Electromyography in Myasthenia Gravis}

\section{J.Y. CHU, V. BRIL, H.G. HUMPHREY, Toronto}

Single fibre electromyographic (SFEMG) studies allow an accurate assessment of neuromuscular function by quantitating jitter (mean consecutive difference of 20 pairs of single fibre potentials) and the percentage of single fibre pairs with neuromuscular blocking. In 20 normal control subjects, mean jitter (MCD) and individual jitter of $90 \%$ of fibre pairs were less than 48 usec, while less than $5 \%$ of fibre pairs showed neuromuscular blocking (NMB) in the ext. digitorum communis muscle (EDC).

44 of 51 patients with myasthenia gravis (MG) were found to have abnormal jitter \pm neuromuscular blocking in EDC. The results in these patients include:

4 of 6 with ocular MG had increased jitter (MCD> 48 usec in $25-55 \%$ of fibre pairs) without NMB

2 of 3 with generalized $M G$ in clinical remission had normal MCD and NMB

18 of 25 with generalized MG controlled on immunosuppressive drugs had MCDs of 32 to $88 \mathrm{usec}$, all had abnormal MCD in more than $10 \%$ of pairs and significant NMB in 4.

7 with untreated generalized MG had increased MCD (68-195 usec) and 6 of 7 had increased NMB $(7-50 \%)$

3 of 4 undergoing plasmapheresis showed a significant decrease in neuromuscular blocking, but no change in MCD following plasma exchange

5 MG suspects without clinical or other evidence of MG had normal SFEMG studies

SFEMG in this study of myasthenic patients reflected abnormal neuromuscular function in $86 \%$ of patients with a close correlation between $\mathrm{NMB}$ and clinical weakness, and MCD and the presumed safety factor at the end plate.

P21.

\section{Familial Oculopharyngeal Muscular Dystrophy with Morphological Features of Inclusion Body Myositis}

\section{R.F. NELSON, V. MONTPETIT, Ottawa}

Oculopharyngeal dystrophy is an uncommon form of adult muscular dystrophy transmitted by dominant inheritance and characterized histologically by a vacuolar myopathy without evidence of inflammation. A French Canadian Family is presented in which 25 members are considered, by history or examination, to suffer from the disease. The signal case is that of a 58-year-old woman with mild dysphagia, ptosis and limita- tion of upward eye movement and mild weakness of the shoulder girdle muscles.

A right deltoid biopsy revealed mild myopathic features with scattered atrophic dark staining angulated fibers and a light infiltrate of mononuclear inflammatory cells. As well, the characteristic sarcoplasmic vacuolar dystrophy hange described by Dubowitz and Brooke was seen. Ultrastructural studies of the "rimmed" vacuoles demonstrated membranous whorls and masses of randomly oriented 14 to $18 \mathrm{~nm}$ filaments which have been considered diagnostic of "inclusion body myositis" (IBM). Nuclear inclusions consisting of $8.5 \mathrm{~nm}$ tubular filaments have been reported in oculopharyngeal dystrophy but the above-mentioned sarcoplasmic masses of filaments have not been described. Since these 14 to $18 \mathrm{~nm}$ filaments have also been recorded in a case of Kugelberg-Welander disease and a case of neuromuscular disorder of uncertain nature, they seem to represent non-specific findings and shed some doubt on the possibility of IBM being a distinct inflammatory myopathy.

P22.

\section{Why Does the Interference Pattern Change in Muscular Dystrophies?}

\section{BRIL, E. STALBERG, P. HILTON-BROWN, Toronto, Uppsala}

A modification of Willison's analysis of the EMG interference pattern (Stalberg 1981) has been used. More than 20 recordings were made during different degrees of contraction from weak to nearly maximal. From epochs of $0.3 \mathrm{~s}$ voltage TURNS and AMPLITUDE were measured and plotted against each other. In patients with muscular dystrophies (limbgirdle, FSH and Duchenne) a significant number of recording sites showed the combination of high turns and low amplitudes, outside the $95 \%$ confidence limits defined from a control group. In order to see whether these changes were mainly caused by drop out of part of the muscle fibres from the motor units, ischaemia and curare experiments were performed in forearm muscles of healthy subjects. During ischaemia, known to produce partial neuromuscular block, TURNS/AMPLITUDE relationship changed in the sense of neuropathy, probably due to the changes in potential shape induced by ischaemia and to an additional partial nerve block. During partial regional curarization both TURNS and AMPLITUDE decreased, but their relationship was mainly unchanged, even when the muscle was nearly totally paralysed. The values did not fall into the area where the data for the myopathy patients were found. Finally, patients with myasthenia were tested before and during maximal effect of Tensilon. There was a variable effect on TURNS/AMPLITUDE but there was no distinct movement from "myopathic" to normal values.

The results seem to indicate that the abnormal interference pattern cannot be due exclusively to drop out of individual muscle fibres in myopathic motor units. Other factors are therefore necessary, e.g. abnormal shape of the MUP with additional spike components, low spike amplitudes, prolonged duration, and increased innervation rate. It seems that for a given strength, the myopathic muscle produces more EMG activity than a normal one treated with curare. This may reflect abnormal mechanical characteristics of the muscle fibres.

\section{P23.}

\section{Mitochondrial Myopathy of Cerebro-Hepato-Renal Syndrome of Zellweger}

\section{H.B. SARNAT, G. MACHIN, H.Z. DARWISH, Calgary}

Qualitative and quantitative alterations in mitochondria have been demonstrated in the central nervous system and liver of infants dying with Zellweger syndrome. These changes may be correlated with some of the metabolic and neurologic abnormalities observed clinically. Such infants also have generalized muscular hypotonia and weakness suggestive of neuropathy or myopathy. We have studied muscle tissue from surgical biopsy and/or autopsy from three infants with this disease, using histologic, histochemical, and electron microscopic techniques. All three cases showed similar findings of 'ragged red' muscle fibers with intermyofibrillar and subsarcolemmal zones of increased oxidative enzymatic activity and increased neutral lipid. Ultrastructurally, these zones contained aggregates of closely 
packed mitochondria which varied greatly in size and morphology of cristae structure. Paracrystalline inclusions were not seen, however. Lipid droplets also were increased in size and number. Histochemical type grouping and grouped atrophy were not found, although many scattered atrophic muscle fibers of both types were present. The diaphragm was more severely involved than skeletal muscles of the extremities, and may explain respiratory failure. Mitochondrial myopathy is an integral part of the cerebro-hepato-renal syndrome.

\section{P24.}

\section{Transitional Sclerosis Treated by Plasmapheresis}

\section{T.J. MURRAY, W. HALLIDAY, V. SANGALANG, Halifax}

Large single demyelinating lesions in the brain have been called transitional sclerosis and regarded as a variant of multiple sclerosis. We present the case of a 22 year old woman with a huge lesion in the left hemisphere, shown on two separate biopsies to be a demyelinating process, and the dramatic improvement with plasmapheresis.

The initial presentation in August, 1981 was with dysphasia, mild hemiparesis, homonymous hemianopia and a marked dominant parietal lobe syndrome. CT scans showed a large irregular lesion involving the posterior half of the left hemisphere with enhancing margins. There was no clinical evidence of other lesions in the CNS and oligoclonal banding was negative in the CSF. There was some improvement in the CT appearance and clinical features with steroids but deterioration occurred after one month. A second course of steroids was not helpful and she became aphasic and hemiparetic. After three weeks of plasmapheresis she was speaking fluently and walking with a cane. There are persisting mild parietal lobe changes and homonymous hemianopia.

The pathology, CT appearance and clinical features of this unusual lesion will be presented and we will speculate on the mechanism of phasmapheresis in this situation.

\section{P25.}

\section{The Longterm Management of Advanced Parkinson's Disease with Bromocriptine}

\section{GRIMES, M. HASSAN, P. GRAY, R. MIRELES, A. KAPOOR, Ottawa}

Thirty three patients (average age 67) with longstanding (mean 9 years), advanced (stage III-IV) Parkinson's disease, who initially tolerated, and responded to Bromocriptine, have been followed on the drug for 12 to 48 months (mean $27 \mathrm{~m}$ ).

These patients were all previous moderate to marked levodopa responders who had developed end of dose deterioration ( 25 patients), dose limiting dyskinesias (26 patients), and/or bradykinesia (19 patients).

Before Bromocriptine, the average daily Sinemet dose was $712 \mathrm{mg}$. The peak effect of Bromocriptine was reached by 3 months at a mean daily dose of $24 \mathrm{mg}$. By this time Sinemet had been reduced an average of $231 \mathrm{mg} /$ day.

Eight patients have maintained their peak effect, without further drug changes for 12 to 48 months (mean $27.5 \mathrm{~m}$ ).

Twenty patients had a fall off from peak effect after an average of 14.2 months. Peak effect was regained for a further 14.8 months by gradually increasing Bromocriptine (4 patients) or Sinemet (7 patients) or both drugs ( 9 patients). Average dosage increases were; Bromocriptine $12 \mathrm{mg} /$ day, Sinemet $187 \mathrm{mg} /$ day.

Five patients, after an average of 11 months of improvement, could not maintain their peak effect despite drug changes.

With cautious introduction, and intermittent dosage adjustment, Bromocriptine can be of longterm benefit to patients with advanced Parkinson's disease. The majority of patients have a gradual late fall off in effect which can frequently be reversed with increases in Bromocriptine and/or Sinemet.
P26.

\section{Effect of Electrical Stimulation of the Spinal Cord on Recovery from Acute Spinal Cord Injury in Rats}

\author{
M.C. WALLACE, C.H. TATOR, Toronto
}

The therapeutic value of electrical stimulation of the spinal cord was studied in rats injured by acute compression of the spinal cord. Twenty adult Wistar rats underwent cord compression at T6-7 by the extradural clip compression technique at a force of $125 \mathrm{gm}$. for 1 minute. After injury, and group randomization, stimulating electrodes were placed extradurally, proximal and distal to the injury site and attached to a small implantable receiver-stimulator. The receiver was secured to the para spinal muscles and implanted subcutaneously overlying the thoracic spine. The animals were maintained in specially designed cages with encircling antennae attached to radiofrequency transmitters. The 10 treatment animals were subjected to a $460 \mathrm{kHz}$ electromagnetic field, pulsed at a frequency of $10 \mathrm{~Hz}$. The receivers converted the pulsed radiofrequency into square wave pulses at the cord electrodes (width $1 \mathrm{msec}$, frequency $10 \mathrm{~Hz}$ ). The 10 control animals were exposed to a similar field but with a frequency below the range of the tuned receiver, and therefore they did not receive the square wave pulse. Clinical recovery was assessed by the inclined plane technique which measures the maximum angle of inclination attained without falling.

After 15 weeks of continuous spinal cord stimulation the inclined plane performance was not significantly different between the two groups (treatment group mean, $44.4 \pm 5.3$; control group mean, $41.7 \pm 7.9$ ). This is the first experimental study of the effect of long term continuous electrical stimulation on spinal cord recovery in mammals. The technology required for achieving continuous stimulation, and the guidelines for future study of this modality are documented.

\section{P27.}

\section{Transplantation of Bovine Adrenal Medullary Cells into Mouse Spinal Cord}

\section{S. DAVID, A.J. AGUAYO, B. LIVETT, Montreal}

Recent experiments using transplants that contain neurons or sheath cells have provided new insights and strategies to study interneuronal and neuron-glial interactions. Intracerebral grafting of embryonic substantia nigra into rats in which the dopaminergic nigro-striatal pathways have been previously destroyed results in the improvement of some of the behavioral disorders in these animals (Björklund et al, Nature 289: 497-499, 1981). Isogenic adrenal medullary cells have been transplanted in rats into the anterior chamber of the eye or CNS (Olson et al, Exp. Neurol. 70: 414426, 1980; Perlow et al, Proc. Natl. Acad. Sci. USA. 77: 5278-5281, 1980).

We report here the successful grafting of xenogenic, bovine, chromaffin cells into the spinal cord of adult C57BL/6J mice. Adrenal medullary cells cultured in vitro (Livette et al, Nature 278: 256-257, 1979) were introduced into the spinal cord using a micropipette. Light and electronmicroscope examination of the spinal cord of these animals 2-3 weeks after the transplantation revealed groups of adrenal medullary cells with catecholamine fluorescence. Although it is not known if the chromaffin cells establish functional connections with the host tissues, they could be a source of transmitters capable of influencing activity locally.

\section{P28.}

\section{Nerve Growth Activities in Cut and Grafted Rat Sciatic Nerves}

\section{P.M. RICHARDSON, T. EBENDAL, Montreal, Uppsala}

Nerve growth activities in rat sciatic nerves were assayed by recording the neuritic outgrowth from chick embryonic autonomic ganglia cultured for 2 days in collagen gels beside freeze-killed endoneurial fragments. Specimens from normal nerves elicited neuritic outgrowth from sympathetic ganglia that was significantly but partially suppressed by antiserum to mouse NGF and also induced outgrowth from ciliary ganglia. 
These observations corroborate previous evidence for the existence of NGF-like activity and at least one other neurite-promoting substance in normal rodent nerves (Riopelle et al, Neurosci. Lett. 25, 311).

Several observations were made in surgically pretreated nerves.

1. In nerve grafts, NGF-like activity was much higher than normal two days after operation.

2. In the distal stump of transected nerves, NGF-like activity was higher than normal after two days but not after seven days.

3. After one or two weeks Wallerian degeneration, non-NGF activity was lower than normal.

4. Neurolysis did not alter the level of nerve growth activities.

In summary, the normal rat sciatic nerve contains two or more soluble substances that stimulated neuritic outgrowth in vitro. The level of these activities in the endoneurium can be altered by previous manipulation of nerves in vivo.

P29.

\section{The Effectiveness of Treatment of Post-Traumatic Spinal Cord Ischemia with Blood Transfusion or Dopamine Infusion}

\section{E.J. DOLAN, C.H. TATOR, Toronto}

Post-traumatic blood flow (SCBF) was assessed after elevating the mean systemic arterial pressure (mSAP) with a blood transfusion or dopamine infusion. Blood flows were measured by the ${ }^{14} \mathrm{C}$-antipyrine autoradiographic method. Animals were injured at $\mathrm{T} 1$ by acute compression of the spinal cord with a clip exerting a $175 \mathrm{~g}$ force for 1 minute.

Five uninjured animals had an overall mSAP of $120.0 \pm 17.0 \mathrm{mmHg}$ and grey and white matter flows of $74.2 \pm 22.3$ and $18.7 \pm 6.7 \mathrm{ml} / 100$ $\mathrm{g} / \mathrm{min}$ respectively at the $\mathrm{T} 1$ level. Injured, untreated animals (5) had mSAP's of $82.5 \pm 14.1 \mathrm{mmHg}$ and grey and white matter flows of $13.3 \pm$ 12.1 and $3.9 \pm 3.9 \mathrm{ml} / 100 \mathrm{~g} / \mathrm{min}$ at the injury site.

Blood transfusion raised the mSAP's to $127.5 \pm 13.7 \mathrm{mmHg}$ in the in jured animals and doubled SCBF in the grey and white matter to $25.6 \pm$ 30.2 and $6.3 \pm 6.4 \mathrm{ml} / 100 \mathrm{~g} / \mathrm{min}$ respectively. Dopamine was not as beneficial raising the mSAP to $101.0 \pm 16.7 \mathrm{mmHg}$ and grey and white matter flows to $18.4 \pm 12.4$ and $5.8 \pm 5.9 \mathrm{ml} / 100 \mathrm{~g} / \mathrm{min}$ respectively.

Autoregulation was found to be impaired at the injury site as both blood transfusion and dopamine infusion were able to elevate mSAP after posttraumatic spinal cord injury. Blood transfusion can bring mSAP back to uninjured control levels and double $\mathrm{SCBF}$ in the injured areas. The lesser effect of dopamine on the SCBF reflects the lower mSAP's achieved by this group.

\section{P30.}

\section{Comparison of Techniques for Experimental Spinal Cord Injuries in the Rat}

\section{KHAN, R. GRIEBEL, Saskatoon}

Three techniques were compared in experimental spinal cord injuries in the rat. These include; 1) the weight dropping, 2) the aneurysm clip compression and 3) the extradural balloon compression methods. In principle, different forces are used in technique 1 , while a constant force for different durations is maintained in the techniques 2 and 3 .

The relationship between these types of spinal cord injuries and subsequent recovery, as well as the histological changes were examined. Recovery was assessed weekly for 6 weeks by the incline plane method of Rivlin and Tator.

A total of 185 Sprague Dawley female rats were used. Ten animals had no surgery while ten were subjected to a $5 \mathrm{~mm}$ middorsal myelectomy. These two groups were used to assess the boundry values for maximum and minimum recovery. One hundred animals were subjected to cord injuries by one of the three techniques. Another 10 animals had complete or partial laminectomy without cord injury and served as controls. A final group of 55 animals were subjected to similar spinal cord injuries and sacrificed at varying intervals from time of trauma to 4 hours later to determine early histological changes in the spinal cord. Late histological changes were also noted in animals followed for 6 weeks.
Technique $!$ is unreliable for experimental spinal cord injury in the rat because of gross inconsistencies both in subsequent recovery and early histological changes. The major factor in the pathogenesis of this injury is believed to be mechanical.

Technique 2 results in consistent cord injuries with respect to subsequent recovery although histological changes do not strictly correlate with functional recovery. Both mechanical and vascular factors seem important in the pathogenesis of such injuries.

Technique 3 consistently produced complete recovery with 6 minutes but no recovery with 7 minutes of $0.1 \mathrm{cc}$ air inflated balloon compression of the cord indicating a steep dose response curve. The primary pathogenesis is likely ischemia. However, using a $0.2 \mathrm{cc}$ air inflated balloon no recovery was noted after 1 minute compression, suggesting a mechanical factor in pathogenesis. Early and late histological changes in the spinal cord are still being examined and will be reported.

P31.

\section{Successful Treatment of Collicular Deafness: Removal of a Meningioma of the Quadrigeminal Cistern}

\section{H.S. SMYTH, Toronto}

A 54-year-old male presented with a 6-month history of progressive headache and personality change, a 6-week history of dementia, ataxia, incontinence, and deafness of indeterminate duration. Physical examination disclosed amnesia, disorientation, lethargy, ataxia of gait, retrostasia, dysarthria, left gaze palsy, and Parinaud's syndrome. CT showed dilated lateral ventricles, and invagination of the posterior third ventricle by a spherical, mid-line, contrasting-enhancing tumor of the pineal region.

Three-stage management consisted of: (i) ventriculo-peritoneal shunting, with prompt resolution of hydrocephalus, dementia, ataxia and incontinence; a peculiar auditory inattention was then noted, and the patient described worsening of perceptual deafness, with poor discrimination and lateralization of sound; (ii) percutaneous biopsy, under CT visualization, of the tumor in the pineal region; (iii) complete microsurgical removal, via the subtentorial, supra-cerebellar route, of a $4-\mathrm{cm}$. meningioma and its tentorial origin, decompressing the quadrigeminal plate.

There was immediate post-operative return of normal hearing bilaterally, and complete resolution of the associated Parinaud's syndrome. The patient was discharged nine days post-operatively, returning to work a month later.

The infrequent reports of this rare type of central deafness, and the historical development of this surgical approach to lesions of the pineal region are both reviewed in this paper.

P32.

\section{The Recurrence of Intracranial Meningiomas after Surgical Treatment}

\section{B. ADEGBITE, M. KHAN, Saskatoon}

A study of 114 surgically treated patients with intracranial meningiomas was carried out to evaluate factors influencing recurrence. The date of the initial surgery extended over a 23 year period from January, 1956 to December, 1979. The patients ranged in age from $1 \frac{1}{2}$ years to 82 years. Seventy-one $(62.3 \%)$ were females and $43(37.7 \%)$ were males. The surgical procedure was graded according to Simpson's classification (1957) from $I$ to 5 (grade $1=$ complete excision, grade $5=$ simple decompression). Thirty-three $(28.9 \%)$ were grade 1, fifty-five $(48.2 \%)$ were grade 2 , seven $(6.1 \%)$ were grade 3 , eighteen $(15.8 \%)$ were grade 4 and $1(0.9 \%)$ was grade 5 . There were $8(7 \%)$ postoperative deaths.

Approximately $60 \%$ of the tumors were located in the sphenoid wing (23.7\%), convexity $(21.1 \%)$ and parasagitally (14.9\%). Histologic diagnosis in $96 \%$ of the patients was transitional (42.1\%), synctial $(34.2 \%)$ and fibroblastic $(20.2 \%)$. Eight (7\%) patients received postoperative radiotherapy.

There was evidence of recurrence in $22(19.3 \%)$ patients. Twenty-one underwent a second surgical procedure. Using a life table and survival analysis, it was determined that $80 \%$ of the patients were free of recurrence 5 years after the initial surgery and approximately $48 \% 17$ years after the 
initial surgery. Only the grade of the initial surgery had a statistically significant influence on recurrence. Seventy-eight percent, $72 \%$ and $32 \%$ of grade 1,2 and 4 respectively were free of recurrence 17 years after the initial surgery. Sex of patients, site and histology of the tumor and postoperative radiotherapy had no statistically significant influence on recurrence. Angioblastic and malignant meningiomas were very few (only 4 cases) and recurred relatively quickly.

\section{P33.}

\section{Paraparesis Following Intrathecal Chemotherapy}

\section{A.F. HAHN, J.J. GILBERT, T.E. FEASBY, London}

Transient and permanent paraparesis is reported occasionally after intrathecal injection of anti-cancer agents. A 64 year old man with lymphomatous meningitis was treated repeatedly with lumbar injections of intrathecal cytosine arabinoside and methotrexate dissolved in distilled water. On a single occasion he was given cytosin arabinoside dissolved instead in bacteriostatic water (Squibb Canada), which contains $1.5 \%$ benzyl alcohol as a preservative. Within 10 minutes he developed a complete flaccid paraplegia with anesthesia below T-12. He was placed upright. Lumbar drainage was started by 2 hours and the CSF space rinsed with isotonic saline. He recovered completely 20 minutes later. At a later date, a lumbar injection of the drug using distilled water as the solvent produced no symptoms. At autopsy 6 months after the transient paraplegia, the cauda equina showed fibrous thickening of the perineurium and scattered areas of remyelination in the nerve roots without evidence of axonal degeneration.

Bacteriostatic water applied to rat dorsal roots blocked the compound action potential by 3 minutes. The block was completely reversible when the root was rinsed within 5 minutes, but longer exposure produced incomplete reversibility. Histological examination of rat roots one week after intrathecal injection of bacteriostatic water showed patchy demyelination.

The bacteriostatic water used to dilute intrathecal drugs causes reversible conduction block because of the local anesthetic effect of benzyl alcohol. Prolonged exposure produces demyelination and incomplete recovery. These effects probably explain most instances of paraparesis following intrathecal injections of methotrexate and cytosine arabinoside.

\section{P34.}

Superfractionated Radiation (SF) Versus Superfractionated Radiation in Combination with Misonidazole in the Treatment of Malignant Astrocytomas: A Preliminary Report

\section{P.J. MULLER, K.H. SHIN, R.C. URTASUN, P. GEGGIE,} D. FULTON, Calgary, Edmonton

Radiation therapy delivered in three fractions per day with an interval of $31 / 2$ hours between fractions (SF) is one of the various attempts to improve the local control of malignant astrocytomas. In order to evaluate the effect of $\mathrm{SF}$ alone and $\mathrm{SF}$ in combination with misonidazole over conventional fractionation (CF), a randomized province wide prospective trial was initiated in January 1980 at the Tom Baker Cancer Center, Calgary and the Cross Cancer Institute, Edmonton. By December 1980, 61 patients with histologically verified supratentorial malignant astrocytoma were randomized: 26 to CF (5785 rad/ 30 fractions/ 6 weeks), 17 to SF (6141 rads/ 69 fractions/ 4.5 weeks), and, 18 to SF (6141 rads/ 69 fractions/ 4.5 weeks) in combination with misonidasole ( $1.25 \mathrm{gr} / \mathrm{m}^{`} 23$ times per week).

At the time of this preliminary assessment 22 of the 43 evaluable patients were alive; the overall actuarial survival was $51 \%$. The 6 month actuarial survival rate for CF, SF and SF with misonidozole was $42 \%$, $67 \%$ and $60 \%$ respectively. Although there appeared to be a survival ad vantage of $S F$ over $C F$ no increase in survival was identified with the addition of misonidozole to SF.
P35.

\section{Rebound Nystagmus in Olivocerebellar Atrophy: A Clinicopathological Correlation}

\author{
R.L. BONDAR, J.A. SHARPE, A.J. LEWIS, Toronto
}

Rebound nystagmus (RN) is a transient jerk nystagmus evoked by gaze shifts to midposition, after prolonged eccentric fixation. It beats horizontally in the direction of gaze shift. In darkness, RN is physiological in some normal subjects. When occurring during fixation, this distinctive oscillation is attributed to cerebellar dysfunction. We present the first oculographic and neuropathological correlation of rebound nystagmus in cerebellar degeneration. A 68 year old man had bidirectional RN, paresis of horizontal smooth pursuit, gaze evoked nystagmus, and downbeat nystagmus on lateral gaze, recorded by oculography and cinematography. The RN had linear slow phases, indicating vestibular bias.

Neuropathological study after fatal cerebral hemorrhage showed olivocerebellar atrophy with predominant Purkinje cell loss in the flocculus and lateral hemispheres. The olivary nuclei were severely atrophic but the brainstem tegmentum and pons were otherwise intact. The olivocerebellar pathway has an established role as a visual relay to the flocculus. Flocculectomised monkeys have RN and defective smooth pursuit, as exhibited by our patient. The slow phase bias that generates $\mathrm{RN}$ is opposed to slow phases of gaze evoked nystagmus. RN may be generated in order to stop gaze evoked nystagmus. The visual pursuit system was not the source of the rebound bias, since pursuit was paretic and RN occurs in darkness. Non-retinal signals of eye position from either orbital proprioceptive inflow of internal efference outflow, may generate the rebound bias within the intact vestibular system. This pathological correlation suggests that nonretinal eye position signals are monitored independently of the olivocerebellar circuit. It indicates that $\mathrm{RN}$ is a sign of damage to this cerebellar subsystem.

\section{P36.}

\section{Subacute Necrotic Myelopathy (Foix-Alajouanine): An Immunopathological Study}

\section{KRIEGER, Y. ROBITAILLE, S. CARPENTER, M.B. DELISLE, R. HALL, D. MELANCON, Montreal}

Three patients presented with increasing bilateral leg pain and weakness followed by flaccid paraparesis with loss of sphincter function. An ascending sensory deficit occurred. Fasciculations were sometimes seen in the affected segments. Relentless progression of weakness and sensory loss led to death within three years. In one patient angiography demonstrated an arterior-venous communication in the posterior fossa.

At autopsy all patients demonstrated abnormalities of extramedullary veins with tortuosity, dilatation, and thickening of their walls. Intramedullary capillaries appeared increased in number and often abnormal in structure. Damage to the cord parenchyma was seen in areas where vascular abnormalities were present.

Immunoperoxidase labelled antibody to factor VIII was employed to stain endothelial cells in an attempt to determine whether the intramedullary vessels could be considered as an intrinsic part of a vascular malformation or as secondarily involved. Comparison was made with cerebral arterio-venous malformations and capillary telangiectases.

\section{P37.}

\section{Guillain-Barré Syndrome and Co-Existing Sacral Radiculopathy in a Patient with Herpes Simplex Genitalis}

\section{BLACK, J.D. STEWART, C.A. MELMED, Montreal}

Herpes simplex genitalis infection may cause motor and sensory sacral radiculopathy (SR). Herpes simplex infections may also be followed by a generalized segmental demyelinating peripheral neuropathy, the GuillainBarré syndrome (GBS).

We describe a patient with concurrent SR and GBS, complicating herpes 
simplex genitalis. She presented with severe lower sacral root dysfunction comprising urinary overflow incontinence, obstipation, a lax anal sphincter, and marked perineal sensory loss (SR). Moderate facial diplegia, mildmoderate limb weakness, areflexia and mild peripheral sensory loss were also present (GBS). There were CSF lymphocytosis. Herpes simplex virus was cultured from her cervix.

Sacral radiculopathy associated with herpes simplex genitalis has been attributed to direct invasion of sacral sensory nerves. The cause of the sacral motor dysfunction is not understood. In GBS the segmental demyelination is thought to be immune-mediated. The unique co-existence of these two disorders raises several possibilities regarding their pathogenesis, including:

1. SR may not be the result of direct viral invasion of the sacral roots, but may be due to viral-induced immune-mediated segmental demyelination.

2. The direct invasion of the sacral nerve roots by the virus may produce neural damage which then triggers an immune reaction resulting in GBS.

\section{P38.}

\section{Further Evidence for Peripheral Nerve Involvement in Multiple Sclerosis}

\section{A. EISEN, D.W. PATY, M. HOIRCH, Vancouver}

During the recovery cycle of nerve a sequence of events occurs consisting of refractoriness followed by a supernormal and subnormal period. The whole cycle is completed within $200 \mathrm{msec}$ of a single conditioning pulse and the supernormal period peaks at between $6-7 \mathrm{msec}$ after the conditioning pulse (Stöhr M: Journal of Neurological Sciences 49: 47, 1981). A $6 \mathrm{msec}$ interval between a supramaximal conditioning pulse $\left(\mathrm{S}_{1}\right)$ and submaximal test pulse $\left(\mathbf{S}_{2}\right)$ was therefore considered optimal for investigating supernormality. The submaximal stimulus was sufficient to evoke a response whose amplitude was about $10 \%$ of the response obtained by supramaximal stimulation. Sensory nerve action potentials (SNAPs) evoked by stimulating finger 2 were recorded from the median nerve at the elbow and supernormality was expressed as the SNAP amplitude ratio $\left(\mathrm{S}_{2}\right.$ conditioned $/ \mathrm{S}_{2}$ unconditioned). The results in 40 patients with suspected or definite multiple sclerosis (MS) whose mean age was 36.8 years, were compared to a control group (mean age 37.9 years; $N=25$ ). In the controls the $\left(S_{2}\right.$ conditioned $/ S_{2}$ unconditioned) ratio measured $285 \pm 174$ (range 119 $783)$. In $17(42.5 \%)$ of the patients the $\left(S_{2}\right.$ conditioned/ $S_{2}$ unconditioned) ratio was 100 or less (range $17.4-100$ ). On the other hand, routine sensory nerve action potential characteristics (conduction velocity, amplitude, and dispersion) were normal in the patients, measuring $61.3 \pm 4.2 \mathrm{~m} / \mathrm{sec}, 4.5$ $\pm 2.6 \mathrm{uv}$, and $5.5 \pm 1.1 \mathrm{msec}$ respectively. The failure of supernormal excitability in the patients is considered to reflect a delay in the recovery cycle of peripheral sensory axons. This finding is interesting in that it further supports recently described morphological and physiological abnormalities indicating involvement of the peripheral nervous system in multiple sclerosis.

\section{P39.}

\section{High Dose Pulsed Methylprednisolone in M.S.}

\section{T.J. MURRAY, J. SZERB, Halifax}

A Phase I open trial of high dosed pulsed methylprednisolone therapy was carried out in 30 patients with definite multiple sclerosis. Phase II will be a double blind paired study comparing methylprednisolone and ACTH. Phase I was carried out to determine if there was a reasonable indication of efficacy prior to a larger double blind study.

$1,000 \mathrm{mgs}$. of methylprednisolone was given intravenously over ten minutes on alternate days for five doses. Of 31 trials (one dropout) there were 28 occasions where subjective improvement occurred by the fifth treatment. This consisted of improved gait, increased fatigue levels, return of sensation in the extremities, and improved bowel and bladder function. Improvement was greater than expected without treatment, taken as $50 \%$ in this trial, based on the experience of other trials.

On 24/30 occasions objective improvement was measured. Even accepting a $50 \%$ placebo improvement rate, this represented a significant improvement $\left(x^{2}\right.$ obs $\left.=7.26, p<.01\right)$. The most impressive change in many patients was the improvement in the ability to walk. Of the 18 patients whose major complaint was increasing leg weakness or decrease in balance and/or coordination, 15 were improved in their ability to walk.

The patients with major improvement of their clinical conditions were younger, with less disability and had suffered from the disease for a shorter period of time.

A change in the CSF oligoclonal banding pattern occurred in 8 of the 15 patients. The most improved group showed the highest proportion of changes (4/5) and the highest proportion in which the bands disappeared after therapy (3/5). The subjects with minor improvement had only $2 / 6$ changes in oligoclonal banding.

P40.

\section{Multiple Sclerosis, Optic Neuritis and Diabetes Mellitus: A Three-Way Association?}

\section{S. WARREN, K.G. WARREN, Edmonton}

One hundred multiple sclerosis (MS) patients were compared to normal controls for the occurrence of diabetes mellitus in their family members. This study was undertaken to follow-up a previous one comparing other illnesses among MS patients and their family members to hospital controls and their family members, in which the only association found was between MS and diabetes. The relationship between these two diseases was confirmed. Significantly more MS patients (49\%) than normal controls $(30 \%)$ were diabetic themselves or reported a first or second degree relative (parent, sibling, child, grandparent, aunt or uncle) with diabetes.

Reports of the percentage of optic neuritis $(\mathrm{ON})$ cases who progress to multiple sclerosis range from $10 \%$ to $80 \%$; MS patients who have other first symptoms may at some time experience an attack of $\mathrm{ON}$. The $100 \mathrm{MS}$ patients in our follow-up study were subcategorized as: those whose first symptom was optic neuritis (16\%); those who had experienced ON but not apparently as a first symptom (14\%); and those who had never experienced ON (70\%). Significantly more of the MS patients whose first symptom was optic neuritis $(75 \%), \mathrm{N}=12$ ) were either diabetic themselves or reported a first or second degree relative with diabetes, than MS patients who had never experienced ON $(40 \%, N=28)$. Likewise significantly more $M S$ patients who had experienced $\mathrm{ON}$ whether or not apparently as a first symptom $(70 \%, \mathrm{~N}=21)$ were either diabetic themselves or reported a first or second degree relative with diabetes, than MS patients who had never experienced $\mathrm{ON}(40 \%, \mathrm{~N}=28)$.

It has been hypothesized that $\mathrm{ON}$ and MS are caused by the same agent, and that host susceptibility determines which cases of ON progress to MS. It might be worthwhile to pursue the possibility that a family history of diabetes mellitus is one factor which increases the risk of optic neuritis developing into multiple sclerosis.

\section{P41.}

\section{Ultrastructural Studies of the Origin and Growth of Dense Micro-Spheres in Normal Human Brain}

\section{P. AVERBACK, Montreal}

The dense microsphere (DMS) is a distinctive brain structure which is located in similar topography to the senile plaque of Alzheimer, and which shows significant reduction in frequency per unit volume brain in senility (Averback, Fed. Proc., in press, 1982). The early genesis of the DMS is traced to a process within neuronal parasynaptic mitochondria where the DMS probably evolves from spherical electron densities of 100-250 nm. diameter into the larger 1-10 $\mu$ diameter membrane bound DMS which lack identifiable mitochondrial remnants. DMS in temporal neocortex enlarge by processes of fusion and symmetrical radial growth over the lifespan of the individual (age 0-10 mean volume $7.2 \mu^{3}$; age 20-38, mean volume $12.4 \mu^{3}$, age $65-90$, mean volume $28.5 \mu^{3}$ ). On morphological grounds the alteration, which is found in normal post-infancy human brain, is abnormal, and comparison is made with other types of pathological mitochondrial structural densities. DMS are found in less than $1 \%$ of cells, in only restricted nuclei, and within an even smaller proportion of mitochondria. Large DMS ( $>1 \mu$ diameter) are not present in newborns and large DMS frequency per unit volume of brain is constant in age groups before the eighth decade, which suggests that they are the result of a 
single early life event. The pathological significance of a longterm relatively unchanging structurally stereotyped intracellular body is discussed in relation to immuno-histochemical studies which have shown positive reaction to reovirus antiserum (Averback, Arch. Pathol. Lab. Med., in press, 1981).

\section{P42.}

\section{Seizures in Sleep: Differential Aspects}

\section{G.B. YOUNG, W.T. BLUME, W.C. MERTENS, S. EDER, London, Saskatoon}

Of 1381 patients with epilepsy, seizures occurred exclusively in sleep (ES) in $81(5.9 \%)$, and predominantly in sleep (PS) in $65(4.7 \%)$. Of the remaining 1235 patients in whom sleep did not precipitate attacks, 71 were randomly chosen as controls.

The incidence of grand mal attacks was higher among ES patients $(72 / 81,89 \%)$ and PS patients $(45 / 65,68 \%)$ than in controls $(14 / 71,20 \%)$ $(\mathrm{p}<.001)$. Conversely, complex partial seizures appeared in only $3 / 81$ $(3.8 \%)$ of ES as compared to PS $(15 / 65,24 \%)$ and controls $(25 / 71,35 \%)$ $(\mathrm{p}<.001)$.

At follow-up seizures had ceased or occurred less than once per two months in 62/81 (76\%) in ES patients, but were poorly controlled in 39/65 $(60 \%)$ in PS cases $(p<.001)$. Controls were intermediate.

Neurological examination disclosed abnormalities in 28/65 (43\%) PS but only 18/81 (22\%) ES patients $(p<.01)$.

A lower portion $(25 / 81,31 \%)$ of ES patients had EEG spikes than control $(44 / 71,62 \%)(\mathrm{p} .001)$ with PS patients intermediate. There was no significant difference among the three groups for activation of spikes during sleep. Among children, central-temporal spikes appeared more commonly in ES $(8 / 22,36 \%)$ than PS $(4 / 17,24 \%)$ and controls $(1 / 22,5 \%)$ $(\mathrm{p}<.05)$

\section{P43.}

\section{Free Valproic Acid: Steady State Pharmacokinetics in Children with Seizures}

\section{N. OTTEN, K. HALL, J. IRVINE-MEEK, M. VERMA, M. LEROUX,} D. BRUDNIK, S. SESHIA, Winnipeg

Since VPA is extensively bound to plasma proteins, determination of free drug pharmacokinetic variables may be of therapeutic importance. In view of the paucity of such information, we present the following data from 6 children.

\begin{tabular}{cccccccc}
\hline & $\begin{array}{c}\text { Age } \\
\text { Subject } \\
(\text { Yrs })\end{array}$ & $\begin{array}{c}\mathrm{t}^{1} / 2 \\
\text { (total) } \\
\text { hours }\end{array}$ & $\begin{array}{c}\mathrm{t}^{1} / 2 \\
\text { (free) } \\
\text { hours }\end{array}$ & $\begin{array}{c}\mathrm{VD} \\
\text { (total) } \\
(\mathrm{L} / \mathrm{kg})\end{array}$ & $\begin{array}{c}\mathrm{VD} \\
\text { (free) } \\
(\mathrm{L} / \mathrm{kg})\end{array}$ & $\begin{array}{c}\mathrm{Cl} \\
\text { (total) } \\
\mathrm{ml} / \mathrm{min} / \mathrm{kg}\end{array}$ & $\begin{array}{c}\mathrm{Cl} \\
\text { (free) } \\
\mathrm{ml} / \mathrm{min} / \mathrm{kg}\end{array}$ \\
\hline 1 & 6 & 8.4 & 4.1 & 0.214 & 1.25 & 0.29 & 3.5 \\
2 & 21 & 7.6 & 3.2 & 0.185 & 0.84 & 0.28 & 3.0 \\
3 & 5.5 & 4.6 & 5.5 & 0.166 & 3.45 & 0.42 & 7.3 \\
4 & 15 & 7.3 & 4.8 & 0.171 & 1.36 & 0.27 & 3.3 \\
5 & 11 & 7.4 & 7.5 & 0.147 & 0.84 & 0.23 & 1.3 \\
6 & 16.5 & 9.4 & 4.7 & 0.251 & 1.34 & 0.31 & 3.3 \\
Mean & & 7.5 & 5.0 & 0.189 & 1.51 & 0.30 & 3.6 \\
\pm & & \pm & \pm & \pm & \pm & \pm & \pm \\
S.D. & 1.6 & 1.5 & 0.038 & 0.98 & 0.06 & 2.0 \\
\hline
\end{tabular}

Regression analysis of \% free VPA vs. total VPA demonstrated the following relationship: $\%$ free $=$ total VPA $-13.9(r=0.836)$

$$
5.39
$$

These results show that 1) \% free drug concentration may vary four-fold over the total serum concentration range of $40-120 \mathrm{mcg} / \mathrm{ml}$ thus emphasizing the need for the establishment of a therapeutic range for free drug 2) the clearance of VPA is restrictive and therefore independent of liver blood flow but dependent on changes in the free fraction of the drug. This study was sponsored by the White Cross Guild, MMSFI and CHRF.
P44.

\section{Seizures after Stroke}

\section{S.E. BLACK, V.C. HACHINSKI, J.W. NORRIS, Toronto, London}

The incidence and clinical significance of seizures following stroke remain uncertain, yet they may have implications for diagnosis and management. Clinical data, including documentation of seizures, was collected in a standardised fashion on 827 patients with a completed stroke, consecutively admitted to our Acute Stroke Unit and following for a period of 2-5 years.

There were 744 patients with cerebral infarction (635 hemispheric, 109 brain stem or cerebellar) and 83 with cerebral hemorrhage (71 hemispheric, 12 brain stem or cerebellar). 76 patients had seizures during their first hospital admission or during follow-up (9\%). Seizures were found only in hemispheric lesions, occurring in $11 \%$ of hemispheric infarcts $(68 / 635)$, and in $11 \%$ of hemispheric hemorrhages $(8 / 71)$. They were focal initially in $7 \%(5 / 76)$, focal only in $41 \%(31 / 76)$ and generalised in the rest. They recurred in $18 \%(14 / 76)$, representing $1.6 \%$ of the overall stroke population (14/827). Seizures were most frequent shortly after admission, $42 \%$ occurring by the first day (32/76) and $62 \%$ by the first week $(47 / 76) .87 \%$ of seizures had occurred by the end of the first year $(66 / 76)$.

There was no significant difference in mortality rate during the first admission in the non-seizure group (19\%) compared to patients having seizures in the first week ( $30 \%)$. A potential source of cardiac embolism was present in $27 \%$ of the non-seizure patients with hemispheric infarcts compared to $41 \%$ of patients with seizures by day one, and $38 \%$ with seizures by day eight. This was not statistically significant.

In summary, preliminary analysis of our data indicates that seizures occur only in hemispheric strokes, with similar frequency in cerebral hemorrhage and infarction. The likelihood of seizures in the acute phase is not increased by the presence of a potential cardiac embolic source, and the occurrence of seizures does not affect early mortality.

\section{P45.}

\section{Syntactic Pattern Recognition of Abnormalities in Prolonged Ambulatory Electroencephalograms}

\section{E. SHWEDYK, R. THORNE, J. PATRICK, S.S. SESHIA, Winnipeg}

Syntactic pattern recognition was used to recognize abnormalities in prolonged ambulatory 4 channel electroencephalograms (AEEGs) performed using a cassette recorder. The AEEG was segmented into one second epochs, each epoch being described as one of seven classes. The classes were extracted by choosing a set of features based on the power spectral properties of the AEEG signal. A syntactic grammar was devised to detect events in five categories: 1) Runs of spikes/sharp waves and slow wave; 2) Runs of polyspike or multiple spike activity; 3 ) Isolated spike/sharp waves and slow waves; 4) Isolated spikes/polyspikes/sharp waves and 5) Runs of slow activity. A run was defined as activity $\geq 2$ or more seconds, while isolated activity lasted $<1$ second.

Thirteen, 30-second segments from 6 patients (ages 12-16 years), provided the data base for the method. Computer recognition was compared with the electroencephalographer's visual identification; $69.1 \%$ of the events were identified correctly and there was a $3.1 \%$ false detection rate.

The classification error was reduced by redefining the feature set and classes. The feature set was expanded to include autoregressive model coefficients and inverse filter output properties (eg., prediction error) to better classify transients. The classes were modified to overcome the frequent misclassification of low amplitude spike/sharp wave and slow wave activity along with an excessive detection of low amplitude slow waves. The modification improved the success rate to $90 \%$. Since syntactic based EEG analysis readily incorporates pattern recognition rules employed by electroencephalographers, our preliminary data suggests that this technique might have a practical application in the automatic detection of abnormalities in AEEGs. 
P46.

\section{Postictal Refractoriness Studied in the Amygdaloid (AM) Kindling Preparation}

\section{J.A. WADA, Y. KANEKO, Vancouver}

Thirteen AM kindled cats ( 8 intact, 5 with callosal bisection) were used. We found that following elicitation of Stage 6 seizure (fully kindled AM convulsion) at generalized seizure triggering threshold, a mean interval of 2.8 hours (1-5 hours) is required before evoking another Stage 6 seizures by AM stimulation in intact cats. In contrast, kindled bisected cats required a significantly shorter mean of 1.1 hours ( $20 \mathrm{~min}-2 \mathrm{hrs}$ ) (P 0.05) for elicitation of the second convulsion. In both intact and bisected cats, the duration of the second seizure was much longer than the controlled seizures. When the above refractoriness test was preceded by 5 daily Stage 6 seizures only the bisected cats showed prolongation of mean refractory period from 1.1 to 1.9 hours. In addition, the bisected cats showed reduction rather than prolongation of the second evoked seizures.

When stimulation was applied sequentially between the two kindled AMs contralaterally induced convulsion was suppresed for 2-8 hours in intact animals while bisected cats showed ready precipitation at $1 \mathrm{hr}$. In all the above experiments after discharge was readily observed regardless of whether kindled convulsion was elicited or not.

The above findings suggest that postictal refractoriness following AM kindled convulsion (1) is not localized to the stimulated AM but involves widespread structures, including the contralateral hemisphere, (2) is weakened by callosal bisection but is strengthened by repeated convulsions.

\section{P47.}

\section{Saccadic Intrusions in Internuclear Ophthalmoplegia}

\section{Y.O. HERISHANU, J.A. SHARPE, Beer Sheva, Toronto}

A variety of saccadic oscillations may disrupt fixation in cerebellar, brainstem, or cerebral disease. We describe saccadic oscillations of distinctive waveform in internuclear ophthalmoplegia (INO). Three patients with bilateral INO (two from multiple sclerosis, one from brainstem infarction) had monocular bursts of horizontal ocular tremor that disrupted forward fixation. The instrusions occurred randomly in either eye whether it was fixating or occluded. Infrared oculography showed salvos of abducting saccades at 2 to 6 hertz, each followed by slower adducting return toward the midposition. The return adducting phases were faster than vergence; they had a decreasing velocity exponential trajectory with a mean time constant of 50 msec.

The short time constant of the adducting phase of oscillation militates against simple mismatch between the pulse of innervation that generated the abducting saccade and the step that maintained tonic motor neuron discharge in the midposition. Orbital mechanics dictate a time constant of about $200 \mathrm{msec}$ for slow eye movements caused by pulse-step mismatch. Reversal of the pulse of excitation to intact abducens motor neurons, into a pulse of inhibition, may explain the rapid return toward midposition. Physiological dynamic overshoot of saccades is attributed to such pulse reversal. Lack of medial rectus excitation in INO explains failure of the fellow eye to oscillate conjugately and it explains why the oscillating eye does not return with saccadic velocity, as it does in dynamic overshoot. In contrast to the conjugate saccades of ocular flutter, the oscillations were monocular. These intrusions may arise from disordered control of pause cells, in the midline brainstem tegmentum, that inhibit saccadic pulse generators. These distinctive oscillations probably specify additional involvement outside the medial longitudinal fasciculi in patients with INO.

\section{P48.}

\section{Sanguinous Extracerebral Fluid Collections in Infants}

\section{H. DARWISH, R.H.A. HASLAM, R. JOHNS, Calgary}

Since the advent of CCT, the diagnosis and management of subdural hematoma in infancy has become controversial. The CCT appearance described by Rothenberg and Brandl, has been criticized by Snyder who also asserted further that withdrawal of fluid after a fontanelle puncture does not confirm the presence of a subdural collection. Robertson et al reported spontaneous resolution of similar "subdural collections".

Over the past 3 years, we have encountered 8 infants under 2 years of age in whom a CCT was requested for evidence of acute increase in intracranial pressure. All showed bilateral, asymmetric frontoparietal hypodense areas and widened sulci and interhemispheric fissure. Six of these infants had simultaneous fontanelle and lumbar punctures.

The fontanelle puncture revealed sanguinous fluid with a $\mathrm{Hgb}$ over $2-3$ $\mathrm{gm} \%$, whereas the fluid obtained by simultaneous lumbar puncture was completely clear in 4 , and was tinged with blood with a Hgb less than 1 gm\% in the others.

Child abuse was the suspected etiology leading to acute symptomatology in 6 infants and accidental head trauma in 2. A coagulopathy was excluded in all 8. Seizures were present at onset in 6 and hemiparesis in 2 . Only 1 infant presented with a macrocephaly and 2 had "box shaped" heads. The anterior fontanelle was bulging and tense, and retinal hemorrhages were noted in all 8 patients.

Repeat fontanelle taps were necessary in 5 patients, 3 of whom needed only 2 taps, 1 patient needed 3 and another 4 sequential taps, initially, followed 2 months later when he had a rebleed, with an additional 3 taps.

The CCT showed progressively enlarging extracerebral spaces for an a verage period of 3 months in spite of significant developmental gains and lack of undue velocity of head growth.

When the clinical presentation suggests acute intracranial hemorrhage, and the CCT shows only hypodense areas over the cerebral convexities we recommend diagnostic fontanelle puncture followed by a lumbar puncture.

Repeat fontanelle punctures are indicated only if subsequent undue velocity of head growth, apnea, bradycardia or progressive neurologic deficit occur. The CCT appearance does not reflect the clinical improvement in these patients. It should not be used as an indication for further taps or other neurosurgical adventures.

\section{P49.}

\section{Raccoon Eye Unmasked}

\section{R.G. PERRIN, J. MULLEN, Toronto}

The periorbital haematoma ("raccoon eye") encountered in head injured patients is generally interpreted as indicating a fracture through the anterior base of skull. The culpable fractures are notoriously difficult to visualize with routine skull films and can result in CSF fisual with potentially serious sequelae. The present study was undertaken to determine the anatomical substrate of "raccoon eye".

The characteristic "raccoon eye" appearance was produced in cadaveric specimens by coloured latex injected through "fractures" in the anterior base of skull to approximate the effect of blood tracking from an anterior base of skull fracture. Subsequent dissection of the periorbital region was performed, and the extent and borders of latex diffusion were established.

It appears that the shape and clearly delineated periorbital ecchymosis associated with anterior base of skull correlates with the circumferential attach ment of the orbital septum - and the characteristic coma shaped inferomedial sparing is due to the lacrimal sac.

\section{P50.}

\section{Vertex Epidural Haematomas}

\section{A. GUHA, H. GROSSMAN, R. PERRIN, H. SMYTH, Toronto}

Vertex epidural haematomas, by virtue of their stragetic location, may obstruct both venous outflow and CSF reabsorption. Thus, even small extradural collections may produce critically increased intracranial pressure in head injured patients. Furthermore, as conventional, horizontal CT scanning may fail to disclose vertex midline lesions, there may be considerable delay in the definitive diagnosis of this remediable aspect of the head injury.

Two illustrative cases are presented with CT, angiographic and operative photographic records. In case one, vertex epidural cloth was the only lesion contributing to neurologic deficit, while in case two, evacuation of the lesion proved to be the critical step in the management of a diffuse cerebral injury. The distinguishing clinical features of the lesion, its incidence, and 
recognition, are described. Neuroradiologic assessment of these patients should include coronal CT views, and may require cerebral angiography for exact definition of location and size of the lesions.

\section{P51.}

\section{Head Injury: Barbiturate Failures}

\section{D.W. ROWED, M.L. SCHWARTZ, Toronto}

In the course of a randomized, prospective, cooperative study to compare mannitol vs pentobarbital treatment for intracranial hypertension in head injuries, 35 patients have so far been analyzed. The records of 13 patients treated with pentobarbital and mannitol in a pilot project prior to the randomized trial were also studied. Glasgow Coma Scores were 7 or less in all but three patients at the time ICP monitoring was initiated. All patients were mechanically ventillated and received dexamethasone. Mannitol and/or pentobarbital was administered to those patients with elevation of ICP to greater than 25 torr. In the entire group there were 30 patients who received both mannitol and barbiturate. In 11 patients $(36.6 \%)$ intracranial hematomas had been removed prior to ICP monitoring.

Rapid reduction of ICP to satisfactory levels was initially achieved in virtually all patients but ICP control was not maintained subsequently in 19 patients $(63.3 \%)$. Nineteen patients $(63.3 \%)$ died.

Vigorous controlled mechanical ventillation with maintenance of $\mathrm{PCO}_{2}$ at 25 - 30 torr, early ventricular drainage and avoidance of premature reduction in barbiturate dose appear to be important factors in ICP control. We postulate that some patients suffer such severe mechanical disruption of cerebral tissue that intractable vasogenic cerebral edema and uncontrollable intractable hypertension are the inevitable result. In these patients, barbiturate coma serves only to delay recognition of brain death. Considering the financial and human costs of this type of therapy, we recommend that effort be directed at identifying salvagable patients as the rate of barbiturate failure is alarmingly high among our patients.

\section{P52.}

\section{Pre-Operative and Intra-Operative Electrophysiological Findings in Peripheral Nerve Injuries}

\section{J.D. STEW ART, P.M. RICHARDSON, Montreal}

Electrophysiological techniques are commonly used in the assessment of nerve injuries to establish the presence or absence of intact functioning axons. To determine their reliability we compared pre-operative and intraoperative recordings in patients with suspected complete nerve lesions.

105 patients with blunt peripheral nerve or plexus injuries were studied in the EMG laboratory using standard techniques of motor and sensory (needle electrodes and averaging techniques) nerve conductions, and needle clectromyography. 8 patients appeared to have complete absence of function in the damaged nerve (brachial plexus, 4 ; common peroneal nerve, 2 ; sciatic and ulnar nerves, 1 of each). These were explored 1-10 months after injury. 3 had anatomically complete or almost complete separation of the nerve trunks. Repair with nerve grafts was performed. The others were assessed by nerve stimulation with needle recording of muscle responses and, where possible, nerve action potentials across the lesion. In all 5 cases, there were some preserved or regenerating axons crossing the site of the injury. Thus, pre-operative studies correctly predicted the need for nerve repair in only three of eight patients.

Further refinements of pre-operative electrophysiological tests are therefore advocated. These include motor conduction studies with intramuscular needle recording, and where possible, nerve action potential studies across the lesion using needle electrodes.

\section{P53.}

\section{Digital Nerve Sensory Potentials in Brachial Plexus and Cervical Root Lesion}

\section{M.J. NEWMAN, Winnipeg}

Lesions of the cervical nerve roots and brachial plexus often present difficulties in anatomical diagnosis. A variety of electrophysiological methods has been utilized in such cases, but none is entirely satisfactory. In theory, the amplitude of sensory nerve potentials in the distal part of the limb should give information as to whether a lesion is proximal or distal to the dorsal root ganglion. In practice, the amplitudes obtained are subject to many influences apart from the number of intact large axons present in the nerve, as described by Buchthal in 1966.

Sensory potentials from digital nerves of each finger were measured in 200 normal subjects using a standard orthodromic technique with surface electrodes. The normal range and variability was established for different age and sex groups. Seventy patients with clinically established cervical root or brachial plexus lesions were tested by the same method, including 36 cases of cervical spondylosis with root compression, 20 of trauma, 9 with neuralgic amyotrophy, as well as other lesions.

In spite of the many pitfalls of amplitude measurement it is shown that this can be a useful investigation and can be made more sensitive by calculation of the ratio of amplitudes from the different fingers.

\section{P54.}

\section{Entrapment Ulnar Neuropathy - Surgical Results}

\section{LONG, P. MULLER, R. WEST, Calgary}

Of the mononeuropathies the incidence of ulnar neuropathy is second to only the carpal tunnel syndrome. In the previous 4 years one of the authors has fashioned 41 ulnar procedures in 37 patients, $75 \%$ of whom were male; in the same time interval there were 148 carpal tunnel release procedures in 97 patients $66 \%$ of whom were female. Of the patients with ulnar neuropathy 8 underwent ulnar neurolysis and epicondylectomy and 33 un. derwent neurolysis and anterior transposition. Thirty-eight had preoperative and 28 had post-operative EMG and nerve conduction studies in an average post-operative interval of 7.6 months. There were 28 males and 9 females; the mean age was 48.3 years (range $=14$ to 78 ). In 10 patients the clinical onset of the ulnar neuropathy was sudden. In 3 patients there was an obvious elbow deformity.

Paresthesia in the ulnar distribution were present in $97 \%$ pre-operatively and $18 \%$ post-operatively. Fourty percent had a moderate to severe motor deficit pre-operatively; and, one-third of these improved post-operatively. Thirty percent had moderate to severe wasting pre-operatively; and this improved in one-third post-operatively although no change occurred in those with severe wasting. The pre-operative and post-operative motor conduction velocities were $34.7[\mathrm{SEM}=1.7]$ and $42.3[\mathrm{SEM}=2.2] \mathrm{m} / \mathrm{sec}$ across the elbow respectively $\mid p<0.05]$.

Significant improvement in sensory symptoms and to a lesser extent in motor function occurred in entrapment ulnar neuropathy after neurolysis.

\section{P55.}

\section{Experience with Pergolide Mesylate in Severe Parkinson's Disease}

\section{GRIMES, M. HASSAN, R. MIRELES, P. GAR NER, Ottawa}

Pergolide mesylate, a semisynthetic ergot alkaloid and central dopamine receptor agonist was given to 6 longstanding (mean 14 years) advanced (stage IV) Parkinson's disease patients.

The patients (mean 61 years) had been on levodopa an average of 9.5 years. They all had severe end of dose deterioration and were mobile independently for an average of only 6 (range $0-11$ ) hours daily.

Pergolide was started in hospital at low dose $(0.1 \mathrm{mg}$ daily) and slowly increased over 3 weeks to a mean dose of $2.8 \mathrm{mg}$ (range 1.7-3.6) daily. Four patients had previously been on Bromocriptine. Sinemet was continued, but reduced from previous peak dosage by an average of $206 \mathrm{mg} /$ day. Treatment time has been $6-12$ months (mean $8 \mathrm{~m}$ ).

Five patients have had a marked response with an increase in mobile time to an average of 14.8 (range 12-17) hours daily. Significant improvement in basic parkinsonian features and disability score was noted in all 5 patients. The drug was stopped at 6 months in one patient because of increased dyskinesias. Other side effects were minimal: transient dose related confusional state ( 1 patient), mild initial nausea ( 1 patient). No changes in basic laboratory data were recorded. Despite prior concern, no significant cardiac rhythm changes were recorded on followup Holter Monitor studies. 
Pergolide induced a marked reduction in basal plasma prolactin level in all patients. Dynamic pituitary function testing in 2 patients showed a complete inhibition of prolactin release and blunting of TSH response to TRH. LH response to LHRH was decreased.

Pergolide is a potent dopamine agonist and is markedly effective in patients with advanced fluctuating Parkinson's disease.

\section{P56.}

\section{The Current Pattern of Work-Related Acute Spinal Cord Injuries in Ontario}

C.E.U. EKONG, C.H. TATOR, D.W. ROWED, M.L. SCHW ARTZ, V.E. EDMONDS, Toronto

The current pattern of acute spinal cord injury directly related to occupation in the Province of Ontario is discussed. An earlier review of 358 patients admitted to Toronto General Hospital and Sunnybrook Medical Centre (SMC) between 1948 and 1973 had shown that $29.3 \%$ of the injuries occurred at work. These were the second most frequent cause of acute spinal cord injury - the most common cause being traffic. The present review of patients admitted to SMC between 1974 and 1979 shows that only $17.4 \%$ of the injuries occurred at work. The ages of the patients ranged from 20 to 56 with most of the patients being in the 20 to 29 and 50 to 59 age groups. Ninety-six percent of the patients were males as compared to $78.8 \%$ in the rest of the group. Work-related spinal cord injury was slightly more frequent in the thoracic region when compared with spinal cord injuries from other causes. The most frequent cause of injury was industrial. This is a significant change from the earlier review in which construction was the most common cause of work-related spinal cord injury. The most frequent mechanism of injury in the present study was as a result of falls. The spinal cord injuries sustained were quite severe. Twentyfour percent of the patients had complete cord injury on admission. The mean neurological grade of the patients did not change significantly on discharge. There was an $8 \%$ mortality rate among the work-related group of patients as compared to $11.8 \%$ among the rest of the patients. This was probably related to the higher number of young patients among the workrelated group.

It thus appears that work as a cause of spinal cord injury in Ontario is decreasing in frequency especially among construction workers. This may be related to improved safety measures. However, the frequency of injury and the degree of neurological damage and lack of significant improvement among those injured make continued efforts towards prevention mandatory.

\section{P57.}

\section{Spinal Cord Injury in Elderly Patients}

C.E.U. EKONG, C.H. TATOR, D.W. ROWED, M.L. SCHWARTZ, V.E. EDMONDS, Toronto

Spinal cord injury in elderly patients presents a unique set of problems with regards to the mechanism of injury, the complications, and prognosis. Our experience with 144 consecutive patients with spinal cord injury is discussed. Seventeen of these patients were 60 years of age or older. The age of the patients ranged from 60 to 83 , with a mean age of 68.5 . A comparison of these elderly patients with the younger patients showed that the older patients were more frequently injured in falls whereas the majority of the younger patients were injured in road traffic accidents. A higher proportion of older patients presented with incomplete cord injury $(82.4 \%$ in the elderly as compared to $57.5 \%$ among younger patients). This was probably related to the relatively minor falls that produced the injuries in the older patients. In spite of the better initial neurological status in the older patients, they had a higher death rate $(41.1 \%$ as compared to $7.1 \%$ among those below 60 years of age) and a higher morbidity rate due to respiratory complications. The mean neurological status among the surviving older patients also showed a slight deterioration on discharge.
P58.

Respiratory Insufficiency After High Cervical Percutaneous Cordotomy

\author{
B. TRANMER, W.S. TUCKER, J.M. BILBAO, Toronto
}

Post-operative respiratory insufficiency is a serious, and often fatal complication of high cervical percutaneous radiofrequency cordotomy. It most often follows bilateral cordotomy, but is occasionally seen after unilateral operation, especially if there is pre-existing pulmonary pathology on the side of the patient's pain. The exact mechanism remains uncertain, and abnormalities of the efferent (reticulospinal) and/or afferent (spinoreticular) pathways may play a role.

In the past 5 years, W.S.T. has performed 121 cordotomies in 81 patients. 77 of the patients had pain from cancer. 51 patients had a unilateral cordotomy and 30 patients had bilateral cordotomies as staged procedures, an average of 16.0 days apart (range: $4-60$ days). 6 patients, all with cancer, developed post-operative respiratory insufficiency. 5 had bilateral cordotomy and one patient, with longstanding myelopathy and contralateral brachial plexus invasion by tumour, had only a unilateral cordotomy. 3 of the 6 patients died in their sleep without warning (sleep apnea) 1,2 , and 5 days after the second side cordotomy. In the other 3 cases, severe respiratory insufficiency was temporarily treated by assisted ventilation, but 2 of the patients later died of respiratory failure, and this may have contributed to the death of the remaining patient. Neuropathological study of the cord lesions was possible in 3 cases, but the lesions were not distinguishable from cases without respiratory insufficiency. Our incidence of respiratory insufficiency was $16.7 \%$ after bilateral and $2 \%$ after unilateral percutaneous cordotomy.

P59.

\section{Degenerative Cervical Spondylolisthesis with Myelopathy}

\section{P.W. COOPER, O.B. LERAMO, C.H. TATOR, Toronto}

Cervical spondylosis is a well known condition which may lead to neurological sequelae. Spondylolisthesis of the cervical spine is far less frequent than its lumbar counterpart and may be of congenital or acquired cause.

We have found that in some patients with chronic progressive myelopathy, there is a combination of both spondylosis and spondylolisthesis. In the three year period between 1977 and 1980 six such patients were encountered in which no history of antecedent trauma was obtained.

Surgical intervention was found to be effective in the treatment of the neurological symptoms.

A description of these cases along with a description of the radiological changes is presented. Structural changes in the articular pillars were noted in some cases and their role in the disease process is discussed. Flexion and extension views were very helpful in recognizing the degree of vertebral slippage.

P60.

\section{Lumbar Disc Herniation: A Clinical, Surgical and CT Scan Correlation}

R. GRIEBEL, M. KHAN, S. TCHANG, G. VARUGHESE, Saskatoon

A series of 80 consecutive patients underwent lumbar spinal surgery for suspected disc herniation. The diagnosis was based on clinical examination and all patients underwent preoperative CT scanning of the lumbar spine. The presence of disc herniation was confirmed during surgery in more than $90 \%$ of those patients having a positive CT scan finding. The technique, advantages and limitations of CT scanning in the diagnosis of lumbar disc disease will be discussed. 
The majority of these patients were initially subjected to myelography, but recently this investigation has only been performed for specific indications. A comparison of these two modalities will also be made.

We suggest that CT scanning is the investigation of choice in assessment of patients with clinical features of lumbar disc herniation, providing the surgeon with more accurate and detailed information than myelography.

\section{P61.}

\section{Selective Posterior Rhizotomy in the Treatment of Spasticity}

\section{G.E. OUAKNINE, Montreal}

Division of the posterior roots of the cauda equina was first performed by FOERSTER in 1908 for the treatment of spasticity in Little's disease. A modification of this operation, the Selective Posterior Rhizotomy (S.P.R.), was performed in 90 cases of spasticity (cerebral palsy: 38 cases; trauma: 22 cases; multiple sclerosis: 24 cases; degenerative diseases: 6 cases). Age at operation ranged from 5 to 67 and the follow-up from 1 to 12 years.

The S.P.R. consists of the microsurgical partial resection of each rootlet of the posterior roots from $\mathrm{L}-1$ to $\mathrm{S}-1$ with preservation of the minute radicular arteries. The selective resection (from $1 / 2$ to $2 / 3$ ) of the "spastic rootlets" depends on the muscular testing and preoperative electrophysiological stimulation. The diminution of the nociceptive afferents reduced the spasticity in the corresponding muscular territory and also, unexpectedly, in the suprasegmental level.

A spectacular diminution of the spasticity of the lower limbs was observed in every case and painful spasms disappeared in $90 \%$ of the cases. Movements became more free allowing improvement of the sitting position and of the standing position and gait. Amelioration of a neurogenic bladder was obtained in 17 cases. In patients observed up to 12 years, these results have persisted in $80 \%$ of cases.

Amelioration of spasticity at the level of the upper limbs was noticed in 27 cases, permitting improvement both in writing and in feeding. Speech improved in 13 cases, becoming more understandable. This paradoxical amelioration at the supra-segmental level has permitted a better social reinsertion and rises an interesting neurophysiological problem.

The S.P.R. is a benign operation without mortality or trophic and motor complications. Hypoesthesia, not perceived by the patient and reversible in a few weeks, is sometimes noticed. Only 3 patients with extensive rhizotomy were left with persistant hypoesthesia. There are no sphincter disturbances if the rhizotomy does not extend beyond S-1. However rapidly reversible, bladder incontinence was observed in 4 cases. Contraindications are bad general condition, severe mental retardation and abnormal movements which could be increased.

\section{P62.}

\section{Cervical Fusion with Parham B ands and Acrylic}

\section{J. STRATFORD, Montreal}

Spinal fusion using stainless steel plates or wires with added acrylic is an accepted procedure to provide immediate stabilization and permit early ambulation, particularly in older and debilitated patients (Hansebout and Blomquist, J. Neurosurg. 1980; 53, 606-612). Unfortunately, in some cases, the wires break or pull through the bone they encircle. To lessen this possibility it is suggested that stainless steel Parham bands be used to encircle the laminal arches where needed; these bands are tightened and then twisted to form a "corkscrew" over which acrylic is placed to provide immediate fusion.

Parham bands, named after a New Orleans surgeon, are flat strips of metal, approximately $0.5 \mathrm{cms}$. wide, which were designed to encompass and hold together fragments in a comminuted fracture of a long bone. A special twister is used to tighten and bend the Parham band.

In the last two years, six patients (age range 65 to 85 ) have had a cervical fusion done using this technique. Five have had excellent immediate stabilization; one, a patient with severe rheumatoid arthritis, subsequently dislocated as the Parham band pulled through the exceptionally soft laminal arches of $C_{1}$ and $C_{2}$.
P63.

\section{Symptomatic Spinal Arachnoid Cysts}

\section{H.S. SMYTH, R.D. MACDONALD, N.A. RUSSELL, Toronto, Ottawa}

A syndrome of recurrent segmental chest wall pain made worse by recumbency, and variable hypoaesthesia and hypoalgesia of the corresponding segment (s), has been characterized in 10 patients. Long tract signs were absent in all cases at presentation.

Seven patients underwent myelography, at which upright films were taken after supine traverse of iodophendylate. Contrast-filled subarachnoid loculations were demonstrated by this technique at single or multiple levels, each of which corresponded exactly with the symptomatic segments, in all seven patients.

In five patients, the symptoms were judged severe enough to warrant exploratory thoracic laminectomy. Loculated arachnoid cysts were found at the designated levels in all cases, and communication of the cysts with the spinal subarachnoid space provided immediate post operative relief in all five cases. Typical pain recurred however in three of the five after five, nine-and twenty-four-month intervals respectively. In the latter, recurrent pain was further complicated by an expanding surgical meningocoele with compressive myelopathy which resolved only after cysto-peritoneal shunting.

The clinical presentation of these cysts, which are believed to originate from the septum posticum, distinguishes them from extradural cysts, arachnoid diverticula of nerve roots, and ventral arachnoid cysts causing myelopathy. Their surgical treatment by simple decompression and communication alone appears unsatisfactory at present.

P64.

\section{Results of Transphenoidal Microsurgery for Acromegaly: Correlation of Radiologic, Biochemical, and Ultrastructural Features in $\mathbf{4 6}$ Cases}

\section{H.S. SMYTH, M. WONG, D.W. KILLINGER, Toronto}

Forty-six patients with biochemically confirmed acromegaly were treated by trans-sphenoidal removal of HGH-cell adenomas between 1973 and 1981 .

Thirty-four patients had one or more lateralizing neuroradiographic findings on plain $x$-ray, tomography, cisternography or angiography. The side lateralized by each technique coincided in all instances, and in all cases accurately lateralized the tumor's location, proved at dissection. Mid-line symmetrical abnormalities were noted in 10 cases with mid-line tumors. Contrast-enhancing tumor was accurately localized in all 19 cases with positive CT findings.

Prompt, sustained normalization of growth hormone was achieved in $92.6 \%$ of enclosed (Stages I and II) tumors: the overall cure rate (Stages I to IV) was $79.4 \%$. Elevated prolactin and HGH levels were both corrected in 9 of 11 patients with mixed HGH-PRL-cell adenomas.

While there was no correlation of ultrastructural granulation patterns with pre-operative $\mathrm{HGH}$ levels, the cure rate for densely granulated tumors was $94.4 \%$, while that for sparsely granulated tumors was only $56.3 \%$.

Diabetes mellitus or impaired glucose tolerance was corrected or improved in $73.3 \%$ of involved patients.

Parallel pituitary functions were improved or unchanged in $83.2 \%$. Surgical mortality was zero, and morbidity was confined to transient CSF leakage in two patients and reduction of pituitary reserve in $16.8 \%$. As the surgical cure rate in previously radiated patients was $57.1 \%$, radiation as a first treatment seems contraindicated except in highly selected cases. Selective trans-sphenoidal tumor removal is the best first treatment currently available for acromegaly.

P65.

\section{Superfractionated Radiotherapy in C57B1/6J Mice}

\section{P.J. MULLER, K.H. SHIN, Calgary}

Post-operative conventional [single daily fraction/ radiotherapy $[\mathrm{CF}]$ in the treatment of malignant cerebral astrocytomas results in significantly 
improved survival, but even at doses which approach brain tolerance radiotherapy is unable to sterilize these tumors and recurrence or progression occurs.

We have assessed the effect of superfractionated radiotherapy [SF] which consists of the administration of three fractions per day at 3-4 hour intervals in normal and subcutaneous ependymoblastoma bearing C57B $1 / 6 \mathrm{~J}$ mice. The radiation was administered by an Eldarado Co-60 teletherapy unit. There were 10 mice per group.

The LD50's and 95\% confidence limits for single treatment, CF and SF cranial irradiation were 1732 (1612-1851), 2546 (2050-2841) and 3662 (2900-4424) rads respectively. The LD50's and 95\% confidence limits for single treatment, CF and SF whole body irradiation were 947 (835-1095), 1365 (1299-1430) and 1534 (1456-1613) rads respectively. SF caused significantly $(p<0.05)$ less lethal toxicity than did $C F$.

The mean time for tumor implanted into the hind limb to increase $50 \%$ in diameter was 6.75 days in 4 control groups. The time to increase $50 \%$ in mean diameter in mice irradiated by $C F$ or $S F$ to a dose of 1750 rads over 5 days was 16.0 and 32.5 days respectively and to a dose of 2250 rads was 32.5 and $>61.0$ days respectively.

We conclude that SF results in a significant increase in the therapeutic ratio in this model system.

\section{P66.}

\section{Does Oral Leucovorin Rescue CNS Folate Deficiency Following High Dose Intravenous Methotrexate?}

\section{J.C. ALLEN, G. ROSEN, H. JUERGENS, B. MEHTA, New York}

Osteosarcoma patients free of CNS metastases are at risk for acquiring leukoencephalopathy after receiving multiple courses of high dose intravenous methotrexate (MTX) $\left(8 \mathrm{gm} / \mathrm{m}^{2}\right)$ followed by oral Leucovorin (LV) rescue (10mg PO q6h for 11 doses, (MTX-LV). LV is converted to reduced folates, the major form of which is 5-methyl-tetrahydrofolate (5MTHFA) and rescues dihydrofolate reductase inhibition by MTX. A prospective study of the adequacy of CNS rescue of MTX biochemical toxicity by oral LV was undertaken in newly diagnosed neurologically normal osteosarcoma patients. Prior to surgical resection of the primary tumor, 10 patients received 4 weekly courses of MTX-LV. During the fourth weekly MTX-LV treatment, 0 and 72 -hour serum and CSF determinations of MTX, 5-MTHFA and LV were made. At 0 hour the 5MTHFA concentration was greater than MTX in all patients in serum and CSF, but $6 / 9$ had persistant low levels of MTX in their serum. No MTX was detectable in the CSF and normal values of 5-MTHFA prevailed in the serum and CSF. At 72 hours, significant elevations in serum 5-MTHFA and MTX were detected in all patients and the serum 5-MTHFA concentration exceeded MTX in 5 of 8 patients. No significant change in CSF 5-MTHFA was observed at 72 hours although low levels of MTX could now be detected in 7 of 9 patients. CSF MTX now exceeded 5-MTHFA in 6 of 9 patients. The failure of oral LV to elevate CNS reduced folate levels at 72 hours in the context of significant levels of CNS MTX may result in intermittent CNS folate deficiency. The clinical and pathological syndrome of leukoencephalopathy may evolve after repeated MTX-LV treatments.

P67.

\section{Computed Tomography (CT) Oriented Stereotactic Rotary Frame}

\section{A.A. PATIL, Minot}

This is an inverted " $U$ " shaped frame with right angled corners, so mounted that it can be adjusted vertically, anteroposteriorly and rotating on an axis formed by its free ends. The sliding probe carrier mounted on the frame can be inclined to the desired angle in the plane of the frame from any point therein. The head is firmly fixed by a head holder with four pins. The frame is first adjusted around the head so as to bring it in the CT plane of the target with its axis of rotation passing through the target and then the probe holder is directed on the target. As the frame and the probe carrier are in the CT plane of the target measurements can be directly obtained from the scanner and confirmation of the probe holder position can be done on a single scan picture. The frame, being a rotary type, can then be rotated to any desired angle without altering the direction of the probe holder with respect to the target. It is thus possible to probe the lesion from any point on the skull.

As the system is constructed of plastic, it can be used within the scanner with minimal artifact, and direct measurements can be obtained without any calculations. This system can be used in any total body scanner with standard features and no special computer programming is necessary. Thus it is a very simple and truly CT oriented system.

Illustrative cases will be shown.

\section{P68.}

\section{Conservative Management of Acute Cerebellar Infarction}

\section{B. ADEBGITE, M. KHAN, Saskatoon}

Acute cerebellar infarction may be large enough to produce a local mass resulting in brain stem compression and/or tonsillar herniation. These cases in the past have required urgent posterior fossa craniectomy and cerebellar resection. We are describing our experience in a prospective study (starting December 1979) with 12 patients that were managed conservatively or with lesser surgical intervention (controlled external ventriculostomy). There were 8 males and 4 females ranging in age from 31 to 82 years. Seven patients had the cerebellar infarct on the right and five on the left.

The type of management was determined by the patient's neurological state and findings on CT scan. Specifically, conservative treatment was utilized in patients who presented with acute vertigo, nausea, vomiting and ataxia with little or no further deterioration and whose CT scan showed a cerebellar infarction with a compressed 4 th ventricle and mild to moderate hydrocephalus. Controlled external ventricular drainage (CEVD) was instituted when there was clinical deterioration and radiological evidence of obstructive hydrocephalus with obliteration of the 4th ventricle. All patients who required CEVD were moribund, comatose with ataxic respirations or acute respiratory arrest prior to external drainage.

Six patients were managed successfully by conservative measures which included the use of steroids and osmotic diurectics. The remaining 6 patients required CEVD, one of whom died from progressive brain stem in farction (the last premortem CT scan revealed absence of mass effect). The remaining 5 patients in this group recovered and are independent.

We suggest that there is a definite place for conservative treatment, that obstructive hydrocephalus is an important factor in the progressive deterioration of patients with cerebellar infarction and that treatment of the hydrocephalus may be a successful and safer alternative to immediate posterior fossa surgery.

P69.

\section{Epilepsy from Cerebral Arteriovenous Malformations}

\section{R. LEBLANC, W. FEINDEL, R. ETHIER, Montreal}

Twenty-seven patients with epilepsy as the only manifestation of a cerebral AVM were seen at the MNI-H from 1973 to 1981. The 9 females and 18 males between the ages of 14 and 54 years (mean 25.2 years) had epilepsy for an average of 3.5 years prior to the diagnosis of AVM. Seven patients ( 6 with a frontal and 1 with a temporal AVM) had primarily generalized seizures, 10 patients ( 7 with a temporal and 3 with a frontal AVM) had partial seizures with complex symptoms, 14 patients ( 8 with a frontal, 4 with atemporal and 2 with a parietal AVM) had partial seizures with elementary symptoms, and 13 patients ( 7 with a temporal, 5 with a frontal and 1 with a parietal AVM) had secondarily generalized seizures. Although there was no history of intracranial hemorrhage 7 patients had negative hemispheric signs (facial asymmetry, hemiatrophy, hyperreflexia and hemianopia), 3 had impaired mentation and 2 were ataxic. The EEG, normal in 10 cases, showed non-epileptiform activity in 8 cases and focal epileptiform activity in 9 cases. Angiographic and or histological examinations demonstrated 8 small (less than $2 \mathrm{~cm}$ in diameter) and 19 large supratentorial AVMs. Fifteen involved the frontal lobe, 10 the temporal lobe, 5 the parietal lobe and 1 the occipital lobe. In 6 cases angiography failed to demonstrate the AVM. Plain CT scanning revealed a high- (13 cases) or low- ( 3 cases) density lesion without a mass effect, and diffuse ( 3 cases) or ipsilateral (5 cases) ventricular enlargement. In 2 cases there was 
enlargement of the ipsilateral sylvian fissure. Infusion CT scanning demonstrated vascular enhancement in $80 \%$ of cases and an abnormal blood vessel in $26 \%$ of cases. Two angiographically occult AVMs showed vascular enhancement with infusion CT scanning, thereby demonstrating the vascular nature of the lesions when angiography had failed to do so. CT scanning is useful in the assessment of patients with a seizure disorder because a surgically amenable lesion will sometimes be demonstrated. An AVM is suspected in a patient with a predominantly partial seizure disorder of recent onset when the CT scan demonstrates a high or low density enhancing lesion without a mass effect, in association with signs of cerebral atrophy such as diffuse non-obstructive ventricular enlargement or enlargement of the ipsilateral ventricle or sylvian fissure. This is especially so if an abnormal blood vessel is demonstrated with the infused study.

P70.

\section{Surgical Management of Carotid-Cavernous Aneurysms: The Role of Progressive Internal Carotid Ligation in the Neck Combined with Extracranial-Intracranial Arterial Bypass}

G. MOHR, S.N. MARTINEZ, J.C. GIROUX, V. JUNCO MORALES, Montreal, Habana

Gérard MOHR, S. Napoléon Martinez, Jacques-Cartier Giroux, Victor Junco Morales. Division of Neurosurgery, Notre-Dame Hospital and Department of Surgery, University of Montreal.

Over the past 25 years, 19 cases of intracavernous aneurysms have been encountered at the Notre-Dame Hospital.

In 17 cases, the initial presenting symptom was unilateral ophthalmoplegia (complete in 12 cases, partial in 5 cases) including 3 cases with sudden carotid-cavernous fistula. Significant headaches were constantly associated with these symptoms. Two cases were incidental discoveries.

There was a striking predominance in females ( 16 females, 3 males) and a high incidence of multiple aneurysms ( 7 cases), sometimes appearing successively over several decades. Giant aneurysms were also found more frequently than in any other location ( 7 cases). The aneurysm was intrasellar in 4 cases, including one case of incidental discovery during a trans sphenoidal procedure for suspected pituitary adenoma. Surgical treatment was applied in 16 instances and consisted of combined extra and intracranial carotid ligation ("trapping-procedure") in 2 cases, transphenoidal wrapping in one case, progressive carotid ligation using the Selverstone-clamp in the neck in 10 cases, proximal carotid ligation combined with ECIC-bypass in 3 cases. One patient with carotid-cavernous fistula experienced "spontaneous treatment" during angiography following anaphylactic shock resulting in pituitary apoplexy and thrombosis of the aneurysm.

The results of the treatment (one operative mortality after trapping procedure) will be discussed: the proximal ligation of the internal carotid artery in the neck remains for us the corner-stone of surgical therapy and will be followed by aneurysmal thrombosis in most cases, including fistulae. However, the high incidence of ischemic complications, including embolization from the aneurysm itself, warrants the prophylactic use of extra-intracranial arterial anastomosis.

The surgical technique of combined ECIC-bypass and proximal carotid ligation will be presented in detail.

P71.

\section{Carotid Endarterctomies - A Doppler Follow Up}

M.I. VILAGHY, M.T. RICHARD, H. HUGENHOLTZ, B.G. BENOIT, N.A. RUSSELL, Ottawa

- 100 carotid arteries were examined in 87 patients, one month to five years following endarterectomy

- Of the 100 vessels examined, 24 were found to have Doppler abnormalities of varying degrees

9 internal carotid arteries were completely occluded

5 endarterectomized vessels displayed a stenosis of $50-98 \%$

10 vessels were found to have abnormalities of varying degrees but of less than $50 \%$ stenosis
-25 of the 87 patients reported symptoms of which:

11 related symptoms referable to the endarterectomized vessel

14 related symptoms not necessarily referable to the endarterectomized vessel

Conclusions:

1. Almost $1 / 4$ of the surgically treated vessels displayed some abnormality one month to five years following endarterectomy.

2. Abnormalities were found in 10 of the 11 patients who were symptomatic from the endarterectomized vessel

3. Abnormalities were found in $8 / 62$ patients who were asymptomatic from the endarterectomized vessel

P72.

\section{Vasopressin and Inappropriate Antidiuresis in Acute Neurovascular Illness}

\section{M.F. MAZUREK, Toronto}

Neurological deterioration following admission to hospital is recognized as a serious problem in patients with acute neurovascular illnesses. The syndrome of inappropriate antidiuresis (SIAD) is a potential factor in this worsening. This report presents data concerning the incidence and clinical significance of SIAD in acute stroke, and documents a disturbance of vasopressin osmoregulation in patients with severe SIAD following subarachnoid haemorrhage.

In a prospective study of 138 consecutive patients satisfying strict entry criteria, 7 of 58 patients with acute completed hemispheric stroke developed definite SIAD, while an additional 7 showed lesser evidence of the disorder. Blinded serial quantitative neurological assessments revealed a close correlation between the development of hyponatraemia and neurological deterioration in the 7 patients with definite SIAD $(r=-.95)$

Vasopressin release was investigated in 3 patients who developed SIAD following subarachnoid haemorrhage. Radioimmunoassay confirmed in each case a failure to suppress vasopressin secretion, despite severe hyponatraemia $(\mathrm{Na}<116 \mathrm{mEq} / \mathrm{L})$ and hyposmolality (pOsm $<245$ $\mathrm{mOsm} / \mathrm{kg}$ ). Sequential measurements of plasma osmolality and plasma vasopressin during therapeutic infusion of hypertonic saline showed that osmotic control of vasopressin had been lost.

These studies suggest that SIAD may contribute to deterioration in a significant minority of acute stroke patients, and that some patients with subarachnoid haemorrhage may lose osmoregulatory control of vasopressin secretion, potentially leading to inappropriate antidiuresis and hyponatraemia.

\section{P73.}

\section{Accuracy of the Kety Autoradiographic Measurement of Cerebral Blood Flow Adapted for Positron Emission Tomography}

\section{P. HERSCOVITCH, M.E. RAICHLE, St. Louis}

The autoradiographic method (AM) of regional cerebral blood flow ( $\mathrm{rCBF}$ ) measurement originally proposed by Kety and colleagues was adapted for use with positron emission tomography (PET). Computer simulations were used to study the accuracy of this model.

Because of the limited spatial resolution of PET, a region of interest will contain a mix of grey and white matter, inhomogeneous in flow and partition coefficient ( ). The resultant error in $\mathrm{rCBF}$, however, is less than $5 \%$. Although the AM requires a monotonic increasing input function to ensure a unique result, the PET adaptation does not because of an additional integration in the operational equation. Input function shape did not affect error due to inhomogeneity. Simulation showed tissue ischemia or abnormal does not add to model error.

Inaccuracy in timing the arterial input function does cause large errors (e.g. $17 \%$ for $2 \mathrm{sec}$ delay) with an infusion input. Errors were less with a bolus input (e.g. 10\%). Infrequent blood sampling (e.g. $5 \mathrm{sec}$ ) affected calculated flow by less than $4 \%$. Propagation of errors in tissue activity measurement was largely independent of flow, reflecting the near linear flow vs. activity relationship.

Thus this analysis shows that the adaptation of the AM should provide accurate quantitation of rCBF with PET. 